\title{
Perfect matchings, rank of connection tensors and graph homomorphisms ${ }^{\dagger}$
}

\author{
Jin-Yi Cai ${ }^{\ddagger}$ and Artem Govorov*æ§ \\ Department of Computer Sciences, University of Wisconsin-Madison, Madison, WI 53706, USA \\ *Corresponding author. Email: hovarau@cs.wisc.edu
}

(Received 23 July 2020; revised 23 February 2021; accepted 21 April 2021; first published online 19 July 2021)

\begin{abstract}
We develop a theory of graph algebras over general fields. This is modelled after the theory developed by Freedman et al. (2007, J. Amer. Math. Soc. 20 37-51) for connection matrices, in the study of graph homomorphism functions over real edge weight and positive vertex weight. We introduce connection tensors for graph properties. This notion naturally generalizes the concept of connection matrices. It is shown that counting perfect matchings, and a host of other graph properties naturally defined as Holant problems (edge models), cannot be expressed by graph homomorphism functions with both complex vertex and edge weights (or even from more general fields). Our necessary and sufficient condition in terms of connection tensors is a simple exponential rank bound. It shows that positive semidefiniteness is not needed in the more general setting.
\end{abstract}

Keywords: perfect matchings; graph homomorphisms; graph parameters; tensor and tensor rank; graph algebras 2020 MSC Codes: Primary 05C25

\section{Introduction}

Many graph properties can be described in the general framework called graph homomorphisms. Suppose $G$ and $H$ are two graphs. A mapping from the vertex set $V(G)$ to the vertex set $V(H)$ is a graph homomorphism if every edge $\{u, v\}$ of $G$ is mapped to an edge (or a loop) of $H$. For example, if $H$ consists of two vertices $\{0,1\}$ with one edge between them and a loop at 0 , then a vertex map $\phi: V(G) \rightarrow\{0,1\}$ is a graph homomorphism iff $\phi^{-1}(1)$ is an independent set of $G$. As another example, if $H=K_{q}$ is a clique on $q$ vertices (no loops), then a vertex map $\phi: V(G) \rightarrow\{1, \ldots, q\}$ is a graph homomorphism iff $\phi$ is a proper vertex colouring of $G$ using at most $q$ colours.

A more quantitative notion is the so-called partition function associated with graph homomorphisms. The idea is that we can consider a fixed $H$ with vertex weights and edge weights, and aggregate all graph homomorphisms from $G$ to $H$, in a sum-of-product expression called the partition function. This expression is invariant under graph isomorphisms, thus expressing a graph property of $G$. This is a weighted counting version of the underlying concept $[6,32,37]$.

More concretely, if each vertex $i \in V(H)$ has weight $\alpha_{i}$ and each edge $\{i, j\}$ of $H$ has weight $\beta_{i, j}$ (non-edge has weight 0 ), then the partition function $Z_{H}(\cdot)$ determined by $H$ is

$$
Z_{H}(G)=\operatorname{hom}(G, H)=\sum_{\phi: V(G) \rightarrow V(H)} \prod_{u \in V(G)} \alpha_{\phi(u)} \prod_{\{v, w\} \in E(G)} \beta_{\phi(v), \phi(w)} .
$$

\footnotetext{
${ }^{\dagger}$ A preliminary version of this paper appeared in the Proceedings of SODA 2019 [16].

${ }^{\ddagger}$ Research supported by NSF CCF-1714275.

${ }^{\S}$ Artem Govorov is the author’s preferred spelling of his name, rather than the official spelling Artsiom Hovarau.

(c) The Author(s), 2021. Published by Cambridge University Press. This is an Open Access article, distributed under the terms of the Creative Commons Attribution licence (http://creativecommons.org/licenses/by/4.0/), which permits unrestricted re-use, distribution, and reproduction in any medium, provided the original work is properly cited.
} 
The partition functions of graph homomorphisms can express a broad class of weighted counting problems. Historically these partition functions also arise in statistical physics, where they play a fundamental role [5]. In classical physics the vertex and edge weights are typically (non-negative) real numbers, but in quantum theory they are complex numbers. But even in classical physics, sometimes a generalization to complex numbers allows a theoretically pleasing treatment. For example, Baxter generalized the parameters to complex values to develop the 'commuting transfer matrix' for the six-vertex model [5]. The book [29] (Section 2.5.2) treats the Hamiltonian of a onedimensional spin chain as an extension of the Hamiltonian of a six-vertex model with complex Boltzmann weights.

Another source of fascination with these objects comes from the classification program for counting problems in complexity theory. In recent years, many far-reaching classification theorems have been proved classifying every problem in a broad class of counting problems as being either computable in polynomial time, or being \#P-hard. This has been proved for graph homomorphisms (GH) $[9,13,24,25,28,30,53]$, for counting constraint satisfaction problems (\#CSP) $[8,10,12,26]$ and for Holant problems $[3,4,15,18,31]$. These theorems are called complexity dichotomies. If we consider problem instances restricted to planar graphs and variables to take Boolean values, there is usually a trichotomy, where every problem is either (1) computable in polynomial time, or (2) \#P-hard on general graphs but computable in polynomial time for planar graphs, or (3) \#P-hard on planar graphs. Counting perfect matchings, including weighted versions, is one such problem that belongs to type (2). The planar tractability of counting perfect matchings is by Kasteleyn's algorithm (a.k.a. FKT algorithm) [33, 34, 52]. Valiant introduced holographic algorithms to significantly extend the reach of this methodology $[11,54,55]$. It is proved that for all \#CSP where variables are Boolean (but constraint functions can take complex values), the methodology of holographic algorithms is universal [14]. More precisely, we can prove that (A) the three-way classification above holds and (B) the problems that belong to type (2) are precisely those that can be captured by this single algorithmic approach, namely a holographic reduction to Kasteleyn's algorithm.

\#CSP are 'vertex models' where vertices are variables, and constraints are placed on subsets of these variables. The partition function of GH can be viewed as a special case of \#CSP where each constraint is a binary function as an edge weight (and for undirected graphs, a symmetric binary function). When vertex weights are present they are unary functions.

In contrast to vertex models, one can consider 'edge models' where each edge is a variable, and constraint functions are placed at each vertex. This is called a Holant problem. ${ }^{1}$ Counting perfect matchings is a Holant problem where the constraint function at each vertex is the EXACT-ONE function. Counting all matchings is a Holant problem with the AT-MOsT-ONE constraint. Other Holant problems include counting edge colourings or vertex disjoint cycle covers. Many problems in statistical physics, such as (weighted) orientation problems, ice models, six-vertex models, etc., are all naturally expressible as Holant problems.

It has been proved [15] that for Holant problems defined by an arbitrary set of complex-valued symmetric constraint functions on Boolean variables, (A) the three-way classification above holds, but (B) holographic reductions to Kasteleyn's algorithm are not universal for type (2); there is an additional class of planar P-time computable problems; these, together with holographic reductions to Kasteleyn's algorithm, constitute a complete algorithmic repertoire for this class. (It is open whether this also holds for non-symmetric constraint functions.)

But this should strike the readers as somewhat ironic. Counting perfect matchings is the problem that Kasteleyn's algorithm solves for planar graphs. However this algorithmic approach is proved universal for type (2) only for vertex models but not for edge models, and yet counting

\footnotetext{
${ }^{1}$ Balázs Szegedy [50] studied 'edge coloring models' which are equivalent to a special case of Holant problems where for each arity $d$ a symmetric vertex function $f_{d}$ is given and placed at vertices of degree $d$. In general, Holant problems allow different (possibly non-symmetric) constraint functions from a set assigned at vertices; see [11].
} 
perfect matchings is a quintessential Holant problem. It is most naturally expressed in the edge model. It is not naturally expressed as a vertex model.

Or can it?

Freedman et al. [27] proved that counting perfect matchings cannot be expressed as the partition function of $\mathrm{GH}$; however, their proof restricts to a definition of partition functions with positive vertex weights and real valued edge weights. More importantly they give a characterization for a graph property to be expressible as such a partition function of GH.

Their characterization consists of two conditions on a connection matrix: a rank condition and a positive semidefiniteness condition. But when we move from $\mathbb{R}$ to the complex field $\mathbb{C}$, this positive semidefiniteness condition breaks down. At a high level, a succinct reason is that for complex matrices $M$, it is not true that $M^{\mathrm{T}} M$ is positive semidefinite. However, partition functions of GH with complex weights are interesting $[1,5,29,35,36,44]$, and natural in the quantum setting. More intrinsically (but less obviously), even if one is dealing with counting problems defined by real weights, complex matrices are essential as holographic transformations. For example, the matrix $Z=\frac{1}{\sqrt{2}}\left[\begin{array}{ll}1 & 1 \\ i & -i\end{array}\right]$ is one of the most important holographic transformations $[11,15]$ in dealing with orientation problems such as the six-vertex model, even when all given weights are real. Note that $Z$ transforms the binary EQUALITY function to DISEQUALITY, which is expressible in the form of signature matrices as: $Z^{\mathrm{T}} I Z=\left[\begin{array}{ll}0 & 1 \\ 1 & 0\end{array}\right]$. Thus the results in [27] do not answer whether counting perfect matchings, and other similar problems naturally expressible as Holant problems (edge models), can be expressed as partition functions of $\mathrm{GH}$ when complex vertex and edge weights are allowed. We note that Schrijver $[46,48]$ gave beautiful characterizations of graph properties expressible as partition functions of $\mathrm{GH}$ with complex edge weights but no vertex weights. Thus effectively the vertex weights are all 1 (and there are also subtle differences in the model which we will discuss in Subsection 4.7). So the expressibility of these Holant problems as (1) with complex $\alpha_{i}$ and $\beta_{i, j}$ remained an open problem.

In this paper we resolve this question. We define the notion of a connection tensor. Then we give a tensor theoretic characterization of when a graph parameter can be expressed as GH over any field. We show that there is only one condition, which is both necessary and sufficient for a graph parameter to be expressible by GH with arbitrary vertex and edge weights, and that is a simple exponential bound on the rank of the connection tensor. Positive semidefiniteness is not required (and would not be meaningful in a general field).

This characterization is purely algebraic. As a consequence we show that counting perfect matchings is not expressible as partition functions of GH over an arbitrary field (over a field of characteristic $p>0$ we count perfect matchings modulo $p$ ). We also prove the same inexpressibility for several other naturally defined Holant problems. Over bounded degree graphs, we prove a sharp threshold for the domain size $(|V(H)|)$ for expressibility, using holographic transformations. While we dispense with their positive semidefiniteness condition, the paper [27] is an inspiration for this work from which we borrow many definitions and ideas.

To handle general vertex weights a significant technical difficulty, we have to overcome following the approach of [27] is the possibility that vertex weights can cancel. In particular, the possibility that the sum of all vertex weights can be 0 creates a non-trivial technical obstacle, and we have to introduce some consequential changes to their proof. To do that, in addition to the algebras of quantum graphs $\mathcal{G}(S)$, we define a second type of algebras of quantum graphs $\mathcal{G}_{\subseteq}(S)$, where $S \subseteq \mathbb{Z}_{+}$is a finite set of labels. In $\mathcal{G}(S)$ the generators are precisely $S$-labelled graphs, whereas in $\mathcal{G}_{\subseteq}(S)$ their label sets can be arbitrary subsets of $S$. We need to do this because the normalization argument from [27] fails in our setting (precisely because the sum of all vertex weights can be 0 ). One technical step involves correctly defining the notion of a projection from one quotient algebra 
Table 1.. Main concepts and sets used in the paper

\begin{tabular}{l}
$\bigoplus_{\mathcal{I}} \mathbb{F}, \mathbb{F}^{\mathcal{I}}, \operatorname{Sym}^{n}\left(\mathbb{F}^{\mathcal{I}}\right), \mathrm{rk}_{s}, T(f, k, n)$ \\
$\mathcal{P} \mathcal{L} \mathcal{G}, \mathcal{P} \mathcal{L} \mathcal{G}_{\subseteq}(S), \mathcal{P} \mathcal{L} \mathcal{G}(S), \mathcal{P} \mathcal{L} \mathcal{G}[k](=\mathcal{P} \mathcal{L} \mathcal{G}([k]))$ \\
$\mathcal{G}, \mathcal{G}_{\subseteq}(S), \mathcal{G}(S), \mathcal{G}[k](=\mathcal{G}([k]))$ \\
$\mathcal{K}, \mathcal{K}_{\subseteq S}, \mathcal{K}_{S}, \mathcal{K}_{[k]}$ \\
$\hat{\mathcal{G}}, \hat{\mathcal{G}}_{\subseteq}(S), \hat{\mathcal{G}}(S), \hat{\mathcal{G}}[k](=\hat{\mathcal{G}}([k]))$ \\
$\tilde{\mathcal{G}}_{\subseteq}(S), \tilde{\mathcal{G}}(S), \tilde{\mathcal{G}}[k](=\tilde{\mathcal{G}}([k]))$ \\
$U_{S}, U_{\emptyset}, U_{k}\left(=U_{[k]}\right), u_{S}=U_{S}+\mathcal{K}, u_{\emptyset}=U_{\emptyset}+\mathcal{K}$ \\
$\pi_{S}: \mathcal{G} \rightarrow \mathcal{G}_{\subseteq}(S), \hat{\pi}_{S}: \hat{\mathcal{G}} \rightarrow \hat{\mathcal{G}}_{\subseteq}(S)$ \\
\hline
\end{tabular}

to another, $\hat{\pi}_{S}: \hat{\mathcal{G}} \rightarrow \hat{\mathcal{G}} \subseteq_{\subseteq}(S)$. It must be onto $\hat{\mathcal{G}} \subseteq_{\subseteq}(S)$ which is not in general the same as $\hat{\mathcal{G}}(S)$, the corresponding quotient algebra without the normalization. (In [27] this $\mathcal{G}_{\subseteq}(S)$ was not needed.) After the appropriate algebraic structures are all in place, now somewhat more elaborate than that of [27], we are able to establish our algebraic characterization of expressibility of a graph property as $\mathrm{GH}$.

An outline of this paper is as follows. Our main theorems are Theorems 3.2 and 3.3. To prove these theorems we need a proper algebraic setting, and these are certain infinite-dimensional algebras, which are vector spaces endowed with a multiplication. These algebras are infinitedimensional because we wish to account for all finite labelled graphs in one structure. Being infinite-dimensional introduces some technical complications. In Section 2 we include some basic notions, mainly regarding tensor spaces. In the context of this paper, the coordinates of these infinite-dimensional vector spaces represent partially labelled graphs in the algebra $\mathcal{G}(S)$ or $\mathcal{G}_{\subseteq}(S)$ (to be defined in Section 5). In Section 3, we introduce the basic definitions of graph algebras and connection tensors of a graph parameter. In Section 4, we show how the tensor theoretic characterization can be used to prove that some graph properties cannot be expressed as GH over any field. The main proof starts in Section 5. In Subsection 5.1, we define the monoid of partially labelled graphs and the algebra of quantum graphs in more detail. We define the algebras of quantum graphs $\mathcal{G}(S)$ and $\mathcal{G}_{\subseteq}(S)$, the ideals $\mathcal{K}_{S}$ and $\mathcal{K}_{\subseteq S}$, and the respective quotients $\hat{\mathcal{G}}(S)$ and $\hat{\mathcal{G}}_{\subseteq}(S)$, all to be formally defined in Subsection 5.1. We also introduce and prove the correctness of the definition of the aforementioned projection $\hat{\pi}_{S}: \hat{\mathcal{G}} \rightarrow \hat{\mathcal{G}}_{\subseteq}(S)$, which arises from the linear map $\pi_{S}: \mathcal{G} \rightarrow \mathcal{G}_{\subseteq}(S)$. As said before, the possibility that the vertex weights sum to 0 does not allow us to perform the corresponding normalization step, and we cannot just simply repeat the proofs from [27] without extending all the definitions systematically. With all the groundwork set, we may finally proceed to the main proof. We show the existence of the basis of idempotents in the quotient algebras $\hat{\mathcal{G}}(S)$ for finite $S \subseteq \mathbb{Z}_{+}$by constructing an isomorphism onto $\mathbb{F}^{r}$ for some $r$ (a composition of two isomorphisms, see Lemma 5.5 and Corollary 5.6) thereby bounding their dimensions as well. After that we are able to proceed similarly to Section 4 from [27], modifying the original proofs as needed.

Table 1 lists the main concepts and sets used in the paper.

There is a substantial body of work related to the partition function of graph homomorphisms $[2,7,17,21,23,38,40-43,45,46,48]$. The book [39] by Lovász and the survey by Borgs et al. [6] provide a good overview of various results. We note that Szegedy [51] showed that counting perfect matchings can be expressed as a suitable limit of the partition functions of graph homomorphisms to a graph on two vertices, so this comes very close to be the partition function of an actual vertex model. We will discuss this in Subsection 4.7 . 


\section{Some basic concepts}

In this paper $\mathbb{F}$ denotes an arbitrary field, also viewed as a one-dimensional vector space over $\mathbb{F}$. The set $\mathbb{F}^{\mathcal{I}}$ consists of tuples indexed by $\mathcal{I}$. We let $\mathbb{Z}_{>0}$ denote the set of positive integers. For any integer $k \geq 0$, let $[k]=\{1, \ldots, k\}$. In particular, $[0]=\emptyset$. For finite $\mathcal{I}=[n]$ we write $\mathbb{F}^{n}$. By operations on components $\mathbb{F}^{\mathcal{I}}$ is an algebra (vector space and a ring). By convention $\mathbb{F}^{0}=\mathbb{F}^{\emptyset}=$ $\{\emptyset\}$, and $0^{0}=1$ in $\mathbb{Z}, \mathbb{F}$, etc. We use $\bigsqcup$ to denote disjoint union. In Subsection 2.1 we briefly state some concepts and results. A more detailed account is given in Section A.

\subsection{Multilinear algebra}

We assume that the reader is familiar with tensors. A main feature in this paper is that we deal with infinite-dimensional spaces and their duals; and this infinite dimensionality causes some technical complications. For example, multilinear functions on a direct product $\prod_{i=1}^{n} V_{i}$ of vector spaces can be naturally identified with the dual space $\left(\bigotimes_{i=1}^{n} V_{i}\right)^{*}$ of linear functions on $\bigotimes_{i=1}^{n} V_{i}$. Moreover, $\bigotimes_{i=1}^{n} V_{i}^{*}$ canonically embeds into $\left(\bigotimes_{i=1}^{n} V_{i}\right)^{*}$ via $\left(\otimes_{i=1}^{n} f_{i}\right)\left(\otimes_{i=1}^{n} v_{i}\right)=\prod_{i=1}^{n} f_{i}\left(v_{i}\right)$. A special case is that $\left(V^{*}\right)^{\otimes n}$ embeds into $\left(V^{\otimes n}\right)^{*}$. If all $V_{i}$ 's are finite dimensional then this embedding is an isomorphism. However, for infinite dimensional $V_{i}$, it is well known that this embedding is not surjective. We describe this in Subsection A.1 of the appendix.

For any symmetric tensor $A \in \operatorname{Sym}^{n}(V)$ we define the symmetric rank of $A$ to be the least $r \geq 0$ for which $A$ can be expressed as

$$
A=\sum_{i=1}^{r} \lambda_{i} \mathbf{v}_{i}^{\otimes n}, \quad \lambda_{i} \in \mathbb{F}, \mathbf{v}_{i} \in V,
$$

and we denote it by $\operatorname{rk}_{\mathrm{S}}(A)$. If there is no such decomposition we define $\operatorname{rk}_{\mathrm{S}}(A)=\infty$. If $\operatorname{rk}_{\mathrm{S}}(A)<$ $\infty$ then in any such expression of $A$ as a sum of $\operatorname{rks}_{\mathrm{S}}(A)$ terms all $\lambda_{i} \neq 0$, all $\mathbf{v}_{i} \neq 0$ and are pairwise linearly independent. In Subsection A.2 we show that if $\mathbb{F}$ is infinite, then $\operatorname{rk}_{S}(A)<\infty$ for all $A \in \operatorname{Sym}^{n}(V)$. We prove all needed technical multilinear algebra statements in Section A.

\subsection{Weighted graph homomorphisms}

We recap the notion of weighted graph homomorphisms [27], but state it for an arbitrary field $\mathbb{F}$.

An $\left(\mathbb{F}\right.$-)weighted graph $H$ is a graph with a weight $\alpha_{H}(i) \in \mathbb{F} \backslash\{0\}$ associated with each node $i$ and a weight $\beta_{H}(i, j) \in \mathbb{F}$ associated with each edge $i j$. For undirected $\mathrm{GH}$, we assume $\beta_{H}(i, j)=$ $\beta_{H}(j, i)$.

Let $G$ be an unweighted graph (with possible multiple edges, but no loops) and $H$ a weighted graph (with possible loops, but no multiple edges). A map $\phi: V(G) \rightarrow V(H)$ is a homomorphism if every edge of $G$ goes to an edge or loop of $H$. In this paper, it is convenient to assume that $H$ is a complete graph with a loop at all nodes by adding all missing edges and loops with weight 0 . Then the weighted graph $\mathrm{H}$ is described by an integer $q=|V(H)| \geq 0$ ( $H$ can be the empty graph), a nowhere zero vector $a=\left(\alpha_{1}, \ldots, \alpha_{q}\right) \in \mathbb{F}^{q}$ and a symmetric matrix $B=\left(\beta_{i j}\right) \in \mathbb{F}^{q \times q}$. In this setting every map $\phi: V(G) \rightarrow V(H)$ is a homomorphism. We assign the weights

$$
\alpha_{\phi}=\prod_{u \in V(G)} \alpha_{H}(\phi(u)), \quad \operatorname{hom}_{\phi}(G, H)=\prod_{u v \in E(G)} \beta_{H}(\phi(u), \phi(v)),
$$

and define

$$
\operatorname{hom}(G, H)=\sum_{\phi: V(G) \rightarrow V(H)} \alpha_{\phi} \operatorname{hom}_{\phi}(G, H) .
$$

When $G$ is the empty graph, i.e. $V(G)=\emptyset$, the only map $\phi: \emptyset \rightarrow V(H)$ is the empty map $\phi=\emptyset$; in that case we have the empty products $\alpha_{\emptyset}=1, \operatorname{hom}_{\emptyset}=1$ and hom $(G, H)=1$. 
If all node weights and edge weights in $H$ are 1, then this is the number of homomorphisms from $G$ into $H$. Without loss of generality we require all vertex weights $\alpha_{H}(i) \neq 0$ since any vertex $i$ with $\alpha_{H}(i)=0$ can be deleted together with all incident edges $i j$ and loops at $i$.

Note that when $H$ is the empty graph, then hom $(G, H)=0$ if $G$ is not the empty graph (because there is no map $\phi: V(G) \rightarrow V(H)$ in this case), and hom $(G, H)=1$ if $G$ is the empty graph (because there is precisely one empty map $\phi=\emptyset$ in this case.) The function $f_{H}=\operatorname{hom}(\cdot, H)$ is a graph parameter, a concept to be formally defined shortly.

\section{Graph algebras}

\subsection{Basic definitions}

An $\mathbb{F}$-valued graph parameter is a function from finite graph isomorphism classes to $\mathbb{F} .^{2}$ For convenience, we think of a graph parameter as a function defined on finite graphs and invariant under graph isomorphism. We allow multiple edges in our graphs, but no loops, as input to a graph parameter. A graph parameter $f$ is called multiplicative, if for any disjoint union $G_{1} \sqcup G_{2}$ of graphs $G_{1}$ and $G_{2}$ we have $f\left(G_{1} \sqcup G_{2}\right)=f\left(G_{1}\right) f\left(G_{2}\right)$.

A $k$-labelled graph $(k \geq 0)$ is a finite graph in which $k$ nodes are labelled by $1,2, \ldots, k$ (the graph can have any number of unlabelled nodes). Two $k$-labelled graphs are isomorphic if there is a label-preserving isomorphism between them. We identify a $(k$-labelled) graph with its $(k-$ labelled) graph isomorphism class. We denote by $K_{k}$ the $k$-labelled complete graph on $k$ nodes, and by $U_{k}$, the $k$-labelled graph on $k$ nodes with no edges. In particular, $K_{0}=U_{0}$ is the empty graph with no nodes and no edges. A graph parameter on a labelled graph ignores its labels.

It is easy to see that for a multiplicative graph parameter $f$, either $f$ is identically 0 or $f\left(K_{0}\right)=1$. Every weighted graph homomorphism $f_{H}=\operatorname{hom}(\cdot, H)$ is a multiplicative graph parameter.

The product of two $k$-labelled graphs $G_{1}$ and $G_{2}$ is defined as follows: we take their disjoint union, and then identify nodes with the same label. Hence for two 0-labelled graphs, $G_{1} G_{2}=G_{1} \sqcup$ $G_{2}$ (disjoint union). Clearly, the graph product is associative and commutative with the identity $U_{k}$, so the set of all isomorphism classes of finite $k$-labelled graphs together with the product operation forms a commutative monoid which we denote by $\mathcal{P} \mathcal{L} \mathcal{G}[k]$.

Let $\mathcal{G}[k]$ denote the monoid algebra $\mathbb{F} \mathcal{P} \mathcal{L} \mathcal{G}[k]$ consisting of all finite formal linear combinations in $\mathcal{P} \mathcal{L} \mathcal{G}[k]$ with coefficients from $\mathbb{F}$; they are called ( $k$-labelled, $\mathbb{F}$-) quantum graphs. This is a commutative algebra with $U_{k}$ being the multiplicative identity, and the empty sum as the additive identity. Later, in Section 5 we will expand these definitions to allow label sets to be arbitrary finite subsets of $\mathbb{Z}_{>0}$.

\subsection{Connection tensors}

Now we come to the central concept for our treatment. Let $f$ be any graph parameter. For all


be identified with $\left(V^{\otimes n}\right)^{*}$, where $V$ is the infinite-dimensional vector space with coordinates indexed by $\mathcal{P} \mathcal{L} \mathcal{G}[k]$, i.e. $V=\bigoplus_{\mathcal{P} \mathcal{L G}[k]} \mathbb{F}$. The entry of $T(f, k, n)$ at coordinate $\left(G_{1}, \ldots, G_{n}\right)$ is $f\left(G_{1} \cdots G_{n}\right)$; when $n=0$, we define $T(f, k, n)$ to be the scalar $f\left(U_{k}\right)$. Furthermore, by the commutativity of the product the arrays $T(f, k, n)$ are symmetric with respect to coordinates, i.e.

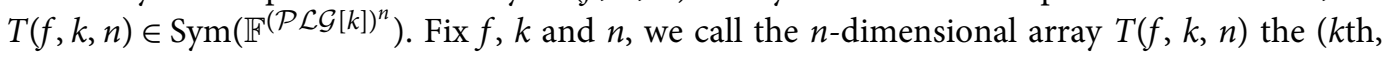
$n$-dimensional) connection tensor of the graph parameter $f$. When $n=2$, a connection tensor is exactly a connection matrix of the graph parameter $f$ studied in [27], i.e. $T(f, k, 2)=M(f, k)$.

In contrast to [27], we will be concerned with only one property of connection tensors, namely their symmetric rank. The symmetric $\operatorname{rank}_{\operatorname{ra}}(f, k, n)=\operatorname{rk}_{S}(T(f, k, n))$, as a function of $k$, $n$, will

\footnotetext{
${ }^{2}$ The concept can be defined over commutative rings but our treatment uses properties of a field.
} 
be called the symmetric rank connectivity function of the parameter $f$. This may be infinite, but for many interesting parameters it is finite, and its growth rate will be important for us.

Remark 3.1. In [27], the matrix rank of $M(f, k)$ was used for the connection matrices. The results of the present paper use the symmetric tensor rank of the connection tensors $T(f, k, n)$ and hold for arbitrary fields. For $n=2$, tensor rank coincides with matrix $\operatorname{rank}$, i.e. $\operatorname{rank}(T(f, k, 2))=$ $\operatorname{rank}(M(f, k))$, and furthermore if char $\mathbb{F} \neq 2$, then the symmetric tensor rank also coincides, $\operatorname{rks}_{S}(T(f, k, 2))=\operatorname{rank}(T(f, k, 2))=\operatorname{rank}(M(f, k))$. Since the field in [27] is $\mathbb{R}$, the notions are consistent.

Proposition 3.1. Let $f$ be a graph parameter that is not identically 0 . The following are equivalent:

(1) $f$ is multiplicative.

(2) $f\left(K_{0}\right)=1$ and for all $n \geq 0, r k_{\mathrm{S}} T(f, 0, n)=1$.

(3) $f\left(K_{0}\right)=1$ and there exists some $n \geq 2, r k_{S} T(f, 0, n)=1$.

Proof. Suppose $f \neq 0$ is multiplicative. Then $f\left(K_{0}\right)^{2}=f\left(K_{0}\right)$, showing that $f\left(K_{0}\right) \in\{0,1\}$. If $f\left(K_{0}\right)=0$, then the relation $f(G)=f(G) f\left(K_{0}\right)$ implies that $f(G)=0$ for every $G$, which is excluded. So $f\left(K_{0}\right)=1$. Trivially $\operatorname{rk}_{S} T(f, 0, n)=1$ for $n=0,1$. Fix any $n \geq 2$. Then $f\left(G_{1} \cdots G_{n}\right)=$ $f\left(G_{1}\right) \cdots f\left(G_{n}\right)$ for any 0-labelled graphs $G_{1}, \ldots, G_{n}$, which implies that $\operatorname{rk}_{S} T(f, 0, n)=1$.

Now suppose $f\left(K_{0}\right)=1$ and for some $n \geq 2, \operatorname{rks}_{\mathrm{S}}(f, 0, n)=1$. This implies that there is a graph parameter $\phi$ and a constant $c_{n}$ such that $f\left(G_{1} \cdots G_{n}\right)=c_{n} \phi\left(G_{1}\right) \cdots \phi\left(G_{n}\right)$. Putting all $G_{i}=K_{0}$, we get $c_{n} \phi\left(K_{0}\right)^{n}=f\left(K_{0}\right)=1$ so $\phi\left(K_{0}\right) \neq 0$ and $c_{n}=1 / \phi\left(K_{0}\right)^{n}$. Dividing $\phi$ by $\phi\left(K_{0}\right)$ we can assume that $\phi$ is normalized so that $f\left(G_{1} \cdots G_{n}\right)=\phi\left(G_{1}\right) \cdots \phi\left(G_{n}\right)$ and $\phi\left(K_{0}\right)=1$. Next, taking $G_{1}=$ $G$ and $G_{i}=K_{0}$ for $2 \leq i \leq n$ we see that $f(G)=\phi(G)$ for every $G$ and therefore $f\left(G_{1} \cdots G_{n}\right)=$ $f\left(G_{1}\right) \cdots f\left(G_{n}\right)$. Finally, substituting $G_{i}=K_{0}$ for $2<i \leq n$, we get $f\left(G_{1} G_{2}\right)=f\left(G_{1}\right) f\left(G_{2}\right)$ so $f$ is multiplicative.

\subsection{Connection tensors of homomorphisms}

Fix a weighted graph $H=(\alpha, B)$. Recall that in the definition of hom $(\cdot, H)$ we assume $H$ to be a complete graph with possible 0 weighted edges and loops, but no 0 weighted vertices. For any $k$-labelled graph $\mathrm{G}$ and mapping $\phi:[k] \rightarrow V(H)$, let

$$
\operatorname{hom}_{\phi}(G, H)=\sum_{\substack{\psi: V(G) \rightarrow V(H) \\ \psi \text { extends } \phi}} \frac{\alpha_{\psi}}{\alpha_{\phi}} \operatorname{hom}_{\psi}(G, H),
$$

where $\alpha_{\phi}=\prod_{i \in[k]} \alpha_{H}(\phi(i))$, and $\alpha_{\psi}$ and hom $\psi$ are defined by (2). Here $\psi$ extends $\phi$ means that if $u_{i} \in V(G)$ is labelled by $i \in[k]$ then $\psi\left(u_{i}\right)=\phi(i)$, so $\frac{\alpha_{\psi}}{\alpha_{\phi}}$ is the product of vertex weights of $\alpha_{\psi}$ not in $\alpha_{\phi}$. Then

$$
\operatorname{hom}(G, H)=\sum_{\phi:[k] \rightarrow V(H)} \alpha_{\phi} \operatorname{hom}_{\phi}(G, H) .
$$

Our main contribution in this paper is that a simple exponential bound in $k$ on the symmetric rank of the connection tensor of a graph parameter characterizes it being expressible as hom $(\cdot, H)$. This holds over all fields $\mathbb{F}$. In the following theorems, the rank function $\operatorname{rk}_{\mathrm{S}}\left(f_{H}, k, n\right)$ is defined over the field $\mathbb{F}$.

Theorem 3.1. For any graph parameter defined by the graph homomorphism $f_{H}=\operatorname{hom}(\cdot, H)$, we have $f_{H}\left(K_{0}\right)=1$ and $r k_{S}\left(f_{H}, k, n\right) \leqslant|V(H)|^{k}$ for all $k, n \geq 0$. 
Proof. The first claim is obvious, as an empty product is 1 , and the sum in (3) is over the unique empty map $\emptyset$ which is the only possible map from the empty set $V\left(K_{0}\right)$. For the second claim notice that for any $k$-labelled graphs $G_{1}, \ldots, G_{n}$ and $\phi:[k] \rightarrow V(H)$,

$$
\operatorname{hom}_{\phi}\left(G_{1} \cdots G_{n}, H\right)=\operatorname{hom}_{\phi}\left(G_{1}, H\right) \cdots \operatorname{hom}_{\phi}\left(G_{n}, H\right) .
$$

When $n=0$, this equality is $\operatorname{hom}_{\phi}\left(U_{k}, H\right)=1$ according to (2), as an empty product is 1 .

By (5) and (6), for the connection tensor $T\left(f_{H}, k, n\right)$ we have the following decomposition:

$$
T\left(f_{H}, k, n\right)=\sum_{\phi:[k] \rightarrow V(H)} \alpha_{\phi}\left(\operatorname{hom}_{\phi}(\cdot, H)\right)^{\otimes n},
$$

where each $\operatorname{hom}_{\phi}(\cdot, H) \in \mathbb{F}^{\mathcal{P} \mathcal{L G}[k]}$ and $k, n \geq 0$. Let $q=|V(H)|$. Then each $T\left(f_{H}, k, n\right)$ is a linear combination of $q^{k}$ tensor $n$-powers and therefore $\operatorname{rk}_{S} T\left(f_{H}, k, n\right) \leq q^{k}$ for $k, n \geq 0$.

The main results of this paper are Theorems 3.2 and 3.3, a converse to Theorem 3.1.

Theorem 3.2. Let $f$ be a graph parameter for which $f\left(K_{0}\right)=1$ and there exists a non-negative integer $q$ such that $r k_{S}(f, k, n) \leq q^{k}$ for every $k, n \geq 0$. Then there exists a weighted graph $H$ with $|V(H)| \leq q$ such that $f=f_{H}$.

More generally, we have the following stronger theorem.

Theorem 3.3. Let $f$ be a graph parameter for which $f\left(K_{0}\right)=1$ and there exists a non-negative integer $q$ such that for every $k \geq 0$ there exists $n \geq 2$ such that $r k_{S}(f, k, n) \leq \min \left(n-1, q^{k}\right)$. Then there exists a weighted graph $H$ with $|V(H)| \leq q$ such that $f=f_{H}$.

Theorem 3.3 implies Theorem 3.2 by choosing a large $\mathrm{n}$. Indeed if $\operatorname{rk}_{\mathrm{S}}(f, k, n) \leq q^{k}$, we may choose any $n \geq \max \left(q^{k}+1,2\right)$, which is $q^{k}+1$ unless $q=0$ and $k>0$.

In Section 5 we will prove Theorem 3.3, then Theorem 3.2 also follows.

\section{Applications}

In this section we give some applications of Theorem 3.1. In Subsection 4.7, we also describe some fine distinctions in the model of graph homomorphism in this paper as is found in [27], compared to that of [46]. In Subsection 4.7, we also use holographic transformations to show that the graph parameter that counts the number of perfect matchings in a graph can be expressed as a limit of partition functions of vertex models, a result of Szegedy [51].

\subsection{Tensor rank lower bound of certain tensors}

We first prove a lemma about the rank of the connection tensor for graph matchings. Let $\mathcal{M}_{a, b}=$ $\mathcal{M}_{n ; a, b} \in \operatorname{Sym}^{n}\left(\mathbb{F}^{2}\right)$ denote the function $\{0,1\}^{n} \rightarrow \mathbb{F}(n \geq 0)$, such that on the all-0 input $\mathbf{0}$ it takes value $a$, on all inputs of Hamming weight one it takes value $b$, and on all other inputs it takes value 0 . This function is denoted by $[a, b, 0, \ldots, 0]$ in the Holant literature. $\left(\mathcal{M}_{0 ; a, b}\right.$ is just a constant $a$. $)$ We have the following lemma; the proof is adapted from the proof of Lemma 5.1 in [20].

Lemma 4.1. If $b \neq 0$ and $n \geq 0$, then $r k_{\mathrm{S}} \mathcal{M}_{n ; a, b} \geq n$.

Proof. For $n=0$ the lemma is trivial. Let $n \geq 1$. Clearly $\mathcal{M}_{n ; a, b} \neq 0$, and so $r=\operatorname{rks} \mathcal{M}_{n ; a, b} \geq 1$. Suppose $r<n$ for a contradiction. Then we can write $\mathcal{M}_{n ; a, b}=\sum_{i=1}^{r} \lambda_{i} v_{i}^{\otimes n}$ where $\lambda_{i} \in \mathbb{F}$, and $v_{i}=\left(\alpha_{i}, \beta_{i}\right) \in \mathbb{F}^{2}$ are non-zero and pairwise linearly independent. The decomposition implies that the linear system $A x=\vec{b}$ with the extended matrix 


$$
\widehat{A}=[A \mid \vec{b}]=\left[\begin{array}{cccc|c}
\alpha_{1}^{n} & \alpha_{2}^{n} & \ldots & \alpha_{r}^{n} & a \\
\alpha_{1}^{n-1} \beta_{1} & \alpha_{2}^{n-1} \beta_{2} & \ldots & \alpha_{r}^{n-1} \beta_{r} & b \\
\alpha_{1}^{n-2} \beta_{1}^{2} & \alpha_{2}^{n-2} \beta_{2}^{2} & \ldots & \alpha_{r}^{n-2} \beta_{r}^{2} & 0 \\
\vdots & \vdots & \ddots & \vdots & \vdots \\
\beta_{1}^{n} & \beta_{2}^{n} & \ldots & \beta_{r}^{n} & 0
\end{array}\right]
$$

has a solution $x_{i}=\lambda_{i}, 1 \leq i \leq r$. Note that $\widehat{A}$ is $(n+1) \times(r+1)$ and $A$ has only $r$ columns. We show that $\operatorname{rank} \widehat{A}=r+1>\operatorname{rank} A$. This is a contradiction. We consider the following two cases.

(1). All $\beta_{i} \neq 0$. Then, by the pairwise linear independence of $v_{i}$, the ratios $\alpha_{i} / \beta_{i}$ are pairwise distinct. Then the last $r$ rows of $A$, i.e. rows $n-r+2$ to $n+1$ form an $r \times r$ Vandermonde matrix of rank $r$. Note that $n+1 \geq n-r+2>2$. By $b \neq 0$, we get an $(r+1) \times(r+1)$ submatrix of $\widehat{A}$ of rank $r+1$ by taking row 2 and the last $r$ rows.

(2). Some $\beta_{i}=0$. Without loss of generality we can assume it is $\beta_{1}$. Again by the pairwise linear independence of $v_{i}$, all other $\beta_{i} \neq 0$, and all $\alpha_{i} / \beta_{i}$ are pairwise distinct for $2 \leq i \leq r$ (vacuously true if $r=1$ ). Then since $b \neq 0$, the submatrix of $\widehat{A}$ formed by taking rows 1,2 and the last $r-1 \geq 0$ rows have rank $r+1$.

We now show that for an infinite field $\mathbb{F}$, we can give a tight upper bound for $\operatorname{rk}_{\mathrm{S}} \mathcal{M}_{n ; a, b}$ where $b \neq 0$ and $n \geq 1$. The existence of a decomposition $\mathcal{M}_{n ; a, b}=\sum_{i=1}^{r} \lambda_{i} v_{i}^{\otimes n}$ where $r \geq 1$, $\lambda_{i} \in \mathbb{F}$ and $v_{i}=\left(\alpha_{i}, \beta_{i}\right) \in \mathbb{F}^{2}(1 \leq i \leq r)$ is equivalent to the statement that system (7) has a solution $x_{i}=\lambda_{i}(1 \leq i \leq r)$. Note that by Lemma 4.1, we must have $r \geq n$.

Assume $\mathbb{F}$ is infinite. We show how to achieve $r=n$ with one exceptional case. First, we set all $\beta_{i}=1$. By comparing with the Vandermonde determinant $\operatorname{det}([A \mid \vec{t}])$ as a polynomial in $t$, where the last column is $\vec{t}=\left(t^{n}, t^{n-1}, \ldots, t, 1\right)^{T}$, we have

$$
\operatorname{det} \widehat{A}=\operatorname{det}([A \mid \vec{b}])=(-1)^{n} \prod_{1 \leq i<j \leq n}\left(\alpha_{i}-\alpha_{j}\right) \cdot\left(a-b \sum_{i=1}^{n} \alpha_{i}\right) .
$$

To see this equation, note that as a polynomial in $t$ the Vandermonde determinant $\operatorname{det}([A \mid \vec{t}])=$ $\prod_{1 \leq i<j \leq n}\left(\alpha_{i}-\alpha_{j}\right) \prod_{i=1}^{n}\left(\alpha_{i}-t\right)=\sum_{i=0}^{n} c_{i} t^{i}$ for some $c_{i} \in \mathbb{F}(0 \leq i \leq n)$. Then $\operatorname{det} \widehat{A}=a c_{n}+$ $b c_{n-1}$.

If we set $\alpha_{1}, \ldots, \alpha_{n} \in \mathbb{F}$ to be pairwise distinct, and $\sum_{i=1}^{n} \alpha_{i}=a / b$, then $\operatorname{rank} A=\operatorname{rank} \hat{A}=$ $n$. The (affine) hyperplane $\Pi: \sum_{i=1}^{n} \alpha_{i}=a / b$ has points away from its intersections with finitely many hyperplanes $x_{i}=x_{j}(i \neq j)$, as long as each of these hyperplanes is distinct from $\Pi$. This is trivially true if $n=1$. Let $n \geq 2$. Under an affine linear transformation we may assume $\Pi$ is the hyperplane $x_{n}=0$ in $\mathbb{F}^{n}$ and we only need to show $\mathbb{F}^{n-1}$ is not the union of finitely many, say $k$, affine hyperplanes. Consider the cube $S^{n-1}$ for a large subset $S \subseteq \mathbb{F}$. The union of these $k$ affine hyperplanes intersecting $S^{n-1}$ has cardinality at most $k|S|^{n-2}<|S|^{n-1}$, for a large $S$.

Each hyperplane $x_{i}=x_{j}(i \neq j)$ is distinct from $\Pi$, except in one case

$$
a=0, n=2 \text {, and char } \mathbb{F}=2 \text {. }
$$

In this exceptional case, we can easily prove that indeed $\operatorname{rk}_{S} \mathcal{M}_{2 ; 0, b}=3$.

We have proved the following.

Lemma 4.2. If $\mathbb{F}$ is infinite, $b \neq 0$ and $n \geq 1$, then $r k_{S} \mathcal{M}_{n ; a, b}=n$ with one exception (8). In that exceptional case, $r k_{\mathrm{S}} \mathcal{M}_{2 ; 0, b}=3$. 
We remark that for any infinite $\mathbb{F}$ not in case (8), for $n \geq 2$ we can achieve $\operatorname{rank} A=\operatorname{rank} \widehat{A}=n$ in (7) by further requiring that all $\alpha_{i} \neq 0$ (in addition to being pairwise distinct, and all $\beta_{i}=1$ ). This is simply to avoid the intersections of $\Pi$ with another finitely many hyperplanes distinct from $\Pi$. Setting $a_{i}=1 / \alpha_{i}$, we can set $a_{i} \in \mathbb{F}$ such that $\operatorname{rank} A=\operatorname{rank} \widehat{A}=n$ for the following $\widehat{A}$.

$$
\widehat{A}=[A \mid \vec{b}]=\left[\begin{array}{cccc|c}
1 & 1 & \ldots & 1 & a \\
a_{1} & a_{2} & \ldots & a_{n} & 1 \\
\vdots & \vdots & \ddots & \vdots & \vdots \\
a_{1}^{n-1} & a_{2}^{n-1} & \ldots & a_{n}^{n-1} & 0 \\
a_{1}^{n} & a_{2}^{n} & \ldots & a_{n}^{n} & 0
\end{array}\right] .
$$

The linear system $A x=\vec{b}$ has a solution $\left(\lambda_{1}, \ldots, \lambda_{n}\right)$ implies that $\mathcal{M}_{m ; a, b}=\sum_{i=1}^{n} \lambda_{i} v_{i}^{\otimes m}$, where $v_{i}=\left(1, a_{i}\right)$, for all $0 \leq m \leq n$. (For $m=0, \mathcal{M}_{0 ; a, 1}=a$ is a constant, and $v_{i}^{\otimes 0}=1$.)

\subsection{Perfect matchings}

Let $\mathcal{F}$ be any set of $\mathbb{F}$-valued constraint functions, a.k.a. signatures, from some finite set $[q]$. For example, the binary EQUALITY $\left(=_{2}\right)$ signature on $(x, y)$ outputs value 1 if $x=y$, and 0 otherwise. Similarly one can define ALL-Distinct on $[q]$, and EXACT-ONE and EXACT-TwO on the Boolean domain $(q=2)$. An input to a Holant problem $\operatorname{Holant}(\mathcal{F})$ is $\Omega=(G, \pi)$ where $G=(V, E)$ is a graph (with possible multiple edges and loops), and $\pi$ assigns to each $v \in V$ some $f_{v} \in \mathcal{F}$ of arity $\operatorname{deg}(v)$, and associate its incident edges as input variables to $f_{v}$. The output is $\operatorname{Holant}(G ; \mathcal{F})=$ $\sum_{\sigma} \prod_{v \in V} f_{v}\left(\left.\sigma\right|_{E(v)}\right)$, where the sum is over all edge assignments $\sigma: E \rightarrow[q], E(v)$ denotes the incident edges of $v$ and $\left.\sigma\right|_{E(v)}$ denotes the restriction of $\sigma$. Bipartite $\operatorname{Holant}(G ; \mathcal{F} \mid \mathcal{G})$ are defined on bipartite graphs $G=(U, V, E)$ where vertices in $U$ and $V$ are assigned signatures from $\mathcal{F}$ and $\mathcal{G}$, respectively.

The graph parameter that counts the number of perfect matchings in a graph, denoted by \#PERFECT-MATCHING (or pm), is a quintessential Holant problem, corresponding to the EXACTONE function. In this subsection we show it is not expressible as a GH function over any field. This was proved in [27] for GH functions with real edge and positive vertex weights. However that proof does not work for arbitrary fields, e.g. for the field of complex numbers $\mathbb{C}$, or even for real numbers with arbitrary (not necessarily positive) vertex weights. A crucial condition in [27] is positive semidefiniteness. Our main result (Theorems 3.1, 3.2 and 3.3) indicates that the property of being expressible as a $\mathrm{GH}$ function is completely characterized by tensor rank.

Let $\operatorname{pm}(G)=m \cdot 1 \in \mathbb{F}$ (the sum of $m$ copies of $1 \in \mathbb{F}$ ) where $m$ is the number of perfect matchings in $G$. Obviously, pm is a multiplicative graph parameter with $\operatorname{pm}\left(K_{0}\right)=1$. Next, let $G$ be a $k$-labelled graph, let $X \subseteq[k]$, and let $\operatorname{pm}(G, X)$ denote the number of matchings in $G$ (expressed in $\mathbb{F}$ ) that match all the unlabelled nodes and, for labelled nodes, exactly the nodes in $X$. Then for any $k$-labelled graphs $G_{1}, \ldots, G_{n}$,

$$
\operatorname{pm}\left(G_{1} \cdots G_{n}\right)=\sum_{X_{1} \sqcup \ldots \sqcup X_{n}=[k]} \operatorname{pm}\left(G_{1}, X_{1}\right) \cdots \operatorname{pm}\left(G_{n}, X_{n}\right) .
$$

This means that $T(\mathrm{pm}, k, n)$ is the product $N_{k}^{\otimes n} W_{k, n}$ where $N_{k}$ has infinitely many rows indexed by all $k$-labelled graphs $\mathrm{G}$, but only $2^{k}$ columns indexed by the subsets $X$ of $[k]$, with the entry at $(G, X)$

$$
N_{k ; G, X}=\operatorname{pm}(G, X)
$$


and $W_{k, n}$ is a symmetric $\underbrace{2^{k} \times \ldots \times 2^{k}}_{n \text { times }}$ tensor $\left(\right.$ from $\operatorname{Sym}^{n}\left(\mathbb{F}^{2^{k}}\right)$ ), where

$$
W_{k, n ; X_{1}, \ldots, X_{n}}=\left\{\begin{aligned}
1 & \text { if } X_{1} \sqcup \ldots \sqcup X_{n}=[k], \\
0 & \text { otherwise. }
\end{aligned}\right.
$$

For any $k$, if $W_{k, n}=\sum_{i=1}^{r} a_{i} v_{i}^{\otimes n}$, then $T(\mathrm{pm}, k, n)=\sum_{i=1}^{r} a_{i}\left(N_{k} v_{i}\right)^{\otimes n}$. Hence $\operatorname{rk}_{\mathrm{S}} T(\mathrm{pm}, k, n) \leq$ $\mathrm{rkS}_{S} W_{k, n}$. We show that in fact equality holds. Consider the family of $k$-labelled graphs $\left\{P_{X}\right\}_{X \subseteq[k]}$ of cardinality $2^{k}$ indexed by the subsets of $[k]$ and is defined as follows: each $P_{X}$ has $|X|$ unlabelled vertices $\left\{x_{i}\right\}_{i \in X}$ and $k$-labelled vertices $\left\{y_{i}\right\}_{i=1}^{k}$ labelled 1 to $k$, with an edge between $x_{i}$ and $y_{j}$ iff $i=j$. It is easy so see that for $X, Y \subseteq[k], N_{k ; P_{X}, Y}=1$ if $X=Y$ and 0 otherwise. Then if we consider the subset of rows in $N_{k}$ corresponding to $\left\{P_{X}\right\}_{X \subseteq[k]}$ we see that they form the identity matrix $I_{2^{k}}$ with a suitable order of rows. Therefore $\operatorname{rk}_{S} W_{k, n}=\operatorname{rks}_{S}\left(I_{2^{k}}^{\otimes n} W_{k, n}\right) \leq \operatorname{rks}_{S} T(p m, k, n)$ and so $\mathrm{rks}_{\mathrm{S}} T(\mathrm{pm}, k, n)=\mathrm{rks}_{\mathrm{S}} W_{k, n}$.

Note that for $k=1, W_{1, n}$ is just the perfect matching tensor (or the EXACT-ONE function on $n$ inputs) $\mathcal{M}_{0,1} \in \operatorname{Sym}^{n}\left(\mathbb{F}^{2}\right)$ where $n \geq 1$. Applying Lemma 4.1 with $a=0, b=1$, we get $\operatorname{rk}_{\mathrm{S}} W_{1, n} \geq n$ and therefore $\operatorname{rks}_{\mathrm{S}} T(\mathrm{pm}, 1, n) \geq n$ for $n \geq 1$. Now if pm were expressible as hom $(\cdot, H)$ for some weighted graph $H$ with $q=|V(H)|$, then by Theorem 3.1, $\operatorname{rks}_{\mathrm{S}} T(\mathrm{pm}, k, n) \leq q^{k}$ for $k, n \geq 0$ so that $\operatorname{rks}_{\mathrm{S}} T(\mathrm{pm}, 1, n) \leq q$ for $n \geq 0$. However, as we have just shown $\operatorname{rks}_{\mathrm{S}} T$ (pm, $\left.1, n\right) \geq n$ for $n \geq 1$ which contradicts the upper bound when $n>q$. Hence pm is not expressible as a graph homomorphism function over any field. We state it as a theorem.

Theorem 4.1. The graph parameter \#PERFECT-MATCHING(pm) is not expressible as a graph homomorphism function over any field.

In this proof we have only used simple $k$-labelled graphs that do not have edges between the $k$-labelled vertices. The graphs $\left\{P_{X}\right\}_{X \subseteq[k]}$ clearly have this property, and this property is preserved under product of $k$-labelled graphs. It follows that Theorem 4.1 holds even when pm is restricted to simple graphs.

We can prove the same inexpressibility results for other Holant problems, such as weighted matchings, proper edge colourings, and vertex disjoint cycle covers. We will also discuss bounded degree cases of weighted matchings.

\subsection{Weighted matchings}

We prove that the more general problem \#WEIGHTED-MATCHING $a$ defined by $\operatorname{wm}_{a}(G)=$ Holant $\left(G ;\left\{\mathcal{M}_{n ; a, 1}\right\}_{n \geq 0}\right)$ is not expressible as a GH function over any field, for any $a$.

Clearly, $\mathrm{wm}_{a}$ is a multiplicative graph parameter with $\operatorname{wm}_{a}\left(K_{0}\right)=1$. If $a=0$ then $\mathrm{wm}_{a}$ is just \#PERFECT-MATCHING (pm). Counting all matchings is $\mathrm{wm}_{a}$ for $a=1$.

Let $G$ be a $k$-labelled graph, $X \subseteq[k]$, and let $\mathrm{wm}_{a}(G, X)$ denote the partial Holant sum in $\operatorname{wm}_{a}(G)$ over all $\{0,1\}$-edge assignments of $G$ such that within $[k]$, those in $X$ have exactly one incident edge assigned 1 and all nodes in $[k] \backslash X$ have no incident edges assigned 1 . Then we have for any $k$-labelled graphs $G_{1}, \ldots, G_{n}$,

$$
\operatorname{wm}_{a}\left(G_{1} \cdots G_{n}\right)=\sum_{X_{1} \sqcup \ldots \sqcup X_{n} \subseteq[k]} a^{k-\left|X_{1} \sqcup \ldots \sqcup X_{n}\right|} \operatorname{wm}_{a}\left(G_{1}, X_{1}\right) \cdots \operatorname{wm}_{a}\left(G_{n}, X_{n}\right) .
$$

This means that $T\left(\mathrm{wm}_{a}, k, n\right)$ is the product $N_{k ; a}^{\otimes n} W_{k, n ; a}$, where $N_{k ; a}$ has infinitely many rows indexed by all $k$-labelled graphs $\mathrm{G}$, and $2^{k}$ columns indexed by $X \subseteq[k]$, with the entry at $(G, X)$

$$
N_{k ; a ; G, X}=\operatorname{wm}_{a}(G, X),
$$


and $W_{k, n ; a}$ is a symmetric $\underbrace{2^{k} \times \ldots \times 2^{k}}_{n \text { times }}$ tensor $\left(\right.$ from $\operatorname{Sym}^{n}\left(\mathbb{F}^{2^{k}}\right)$ ), where

$$
W_{k, n ; a ; X_{1}, \ldots, X_{n}}=\left\{\begin{array}{l}
a^{k-\left|X_{1} \sqcup \ldots \sqcup X_{n}\right|} \quad \text { if } X_{1} \sqcup \ldots \sqcup X_{n} \subseteq[k], \\
0 \text { otherwise. }
\end{array}\right.
$$

Hence $\operatorname{rks}_{\mathrm{S}} T\left(\mathrm{wm}_{a}, k, n\right) \leq \mathrm{rk}_{\mathrm{S}} W_{k, n ; a}$. We show that in fact equality holds. Consider the same family of $k$-labelled graphs $\left\{P_{X}\right\}_{X \subseteq[k]}$ defined in the previous subsection. It is easy to see that for $X, Y \subseteq[k], N_{k ; a ; P_{X}, Y}=a^{|X|-|Y|}$ if $Y \subseteq X$ and 0 otherwise. Here by convention, $a^{0}=1$ even if $a=0$. Consider the rows in $N_{k ; a}$ corresponding to $\left\{P_{X}\right\}_{X \subseteq[k]}$. They form the non-singular matrix $\left[\begin{array}{ll}1 & 0 \\ a & 1^{\otimes n}\end{array}\right]$ if the rows and columns are ordered lexicographically for $X, Y \subseteq[k]$. Therefore $\mathrm{rk}_{\mathrm{S}} W_{k, n ; a}=\mathrm{rk}_{\mathrm{S}}\left(\left[\begin{array}{cc}1 & 0 \\ a & 1\end{array}\right]^{\otimes n} W_{k, n ; a}\right) \leq \operatorname{rks}_{\mathrm{S}} T\left(\mathrm{wm}_{a}, k, n\right)$ and so $\operatorname{rk}_{\mathrm{S}} T\left(\operatorname{wm}_{a}, k, n\right)=\operatorname{rk}_{\mathrm{S}} W_{k, n ; a}$.

Note that for $k=1, W_{1, n ; a}=\mathcal{M}_{n ; a, 1}=[a, 1,0, \ldots 0] \in \operatorname{Sym}^{n}\left(\mathbb{F}^{2}\right)$ where $n \geq 1$. Applying Lemma 4.1 with $b=1$, we get $\operatorname{rks}_{S} W_{1, n ; a} \geq n$ and therefore $\operatorname{rkS}_{S} T\left(\operatorname{wm}_{a}, 1, n\right) \geq n$ for $n \geq 1$. Now if $\operatorname{wm}_{a}$ were expressible as hom $(\cdot, H)$ for some weighted graph $H$ with $q=|V(H)|$, then by Theorem 3.1, $\operatorname{rks}_{\mathrm{S}} T\left(\mathrm{wm}_{a}, k, n\right) \leq q^{k}$ for $k, n \geq 0$ so that $\operatorname{rks}_{\mathrm{S}} T\left(\mathrm{wm}_{a}, 1, n\right) \leq q$ for $n \geq 0$. This contradicts $\operatorname{rks}_{S} T\left(\mathrm{wm}_{a}, 1, n\right) \geq n$ when $n>q$. Hence $\mathrm{wm}_{a}$ is not expressible as a graph homomorphism function over any field.

By the same remark for Theorem 4.1, the proof for Theorem 4.2 carries over to simple graphs.

Theorem 4.2. The graph parameter \#WEIGHTED-MATCHING ${ }_{a}\left(w m_{a}\right)$ where $a \in \mathbb{F}$ as a function defined on simple graphs is not expressible as a graph homomorphism function over any field $\mathbb{F}$.

\subsection{Bounded degree graphs}

Fix any $d \geq 2$. A degree- $d$ bounded graph is a graph with maximum degree at most $d$. In this subsection, we investigate the expressibility of the graph parameter \#WEIGHTED-MATCHING $a$ $\left(\mathrm{wm}_{a}\right)$ as a GH function on bounded degree graphs. More precisely, we are interested when $\mathrm{wm}_{a}$ is expressible as a hom $(\cdot, H)$ with $|V(H)|=q$ on degree- $d$ bounded graphs. For convenience, we temporarily allow vertex weights to be 0 , and it will be addressed later.

Given a graph $\mathrm{G}$ (possibly with multiple edges but no loops), let $G^{\prime}$ be the vertex-edge incidence graph of $G$. The vertex set $V\left(G^{\prime}\right)$ consists of the original vertices from $V(G)$ on the LHS and the edges $E(G)$ on the RHS. Let $H$ be the weighted graph specified by vertex weights $\left(\alpha_{1}, \ldots, \alpha_{q}\right) \in \mathbb{F}^{q}$ and a symmetric matrix $B=\left(\beta_{i j}\right) \in \mathbb{F}^{q \times q}$ for edge weights, then for any $G$

$$
\operatorname{hom}(G, H)=\operatorname{Holant}\left(G^{\prime} ;\left\{\sum_{i=1}^{q} \alpha_{i} e_{q, i}^{\otimes n}\right\}_{n \geq 0} \mid B\right) \text {, }
$$

where $\left\{e_{q, i}\right\}_{i=1}^{q} \in \mathbb{F}^{q}$ has a single 1 at the $i$ th position and 0 elsewhere. Here hom $(G, H)$ is expressed in (10) as a domain- $q$ Holant sum on $G^{\prime}$ : any LHS vertex of $G^{\prime}$ of degree $n$ is assigned the signature $\sum_{i=1}^{q} \alpha_{i} e_{q, i}^{\otimes n}$ (which takes value $\alpha_{i}$ if all incident edges have value $i \in[q]$, and 0 otherwise), and any RHS vertex of $G^{\prime}$ (an edge of $G$ ) is assigned the symmetric binary signature specified by $B$.

First, we show that for any $a \in \mathbb{F}$, if $\mathrm{wm}_{a}$ is expressible as hom $(\cdot, H)$ with $|V(H)|=q$ on degree$d$ bounded simple graphs over any field $\mathbb{F}$, then $q \geq d$. Recall the proof from Section 4.3. This time we restrict the connection tensor $T\left(\mathrm{wm}_{a}, 1, d\right)$ (for $\left.k=1\right)$ to the 1-labelled graphs $P_{\emptyset}$ and $P_{[1]}$, which are just $K_{1}$ and $K_{2}$ without the label. The product of $d$-labelled graphs from $\left\{P_{\emptyset}, P_{[1]}\right\}$ 
having $\ell$ copies of $P_{[1]}$ is the star graph $S_{\ell}$ with one internal node labelled by 1 and $\ell$ external unlabelled nodes. All these are degree- $d$ bounded simple graphs. Note that $T\left(\mathrm{wm}_{a}, 1, d\right)_{\mid\left\{P_{\emptyset}, P_{[1]}\right\}^{d}}=$ $\left[\begin{array}{ll}1 & 0 \\ a & 1\end{array}\right]^{\otimes d} W_{1, d ; a}$. Therefore $\operatorname{rk}_{\mathrm{S}}\left(T\left(\mathrm{wm}_{a}, 1, d\right)_{\mid\left\{P_{\emptyset}, P_{[1]}\right\}^{d}}\right)=\operatorname{rk}_{\mathrm{S}}\left(\left[\begin{array}{cc}1 & 0 \\ a & 1\end{array}\right]^{\otimes d} W_{1, d ; a}\right)=\operatorname{rk}_{\mathrm{S}} W_{1, d ; a} \geq$ $d$, the last step is by Lemma 4.1. On the other hand, if $\mathrm{wm}_{a}$ is expressible as hom $(\cdot, H)$ with $|V(H)|=q$ on degree- $d$ bounded simple graphs, then arguing similarly to the proof of Theorem 3.1 but restricting the domain of the arguments $G_{i}, 1 \leq i \leq d$ in (6) to $\left\{P_{\emptyset}, P_{[1]}\right\}$, we have $\mathrm{rk}_{\mathrm{S}}\left(T\left(\mathrm{wm}_{a}, 1, d\right)_{\mid\left\{P_{\emptyset}, P_{[1]}\right\}^{d}}\right) \leq q$ so $d \leq q$. Then clearly this bound also holds if we do not allow 0 -weighted vertices.

Now let $\mathbb{F}$ be infinite. By the remark after Lemma 4.2, if we are not in the exceptional case (8) (we put $n=d$ ), then for some $a_{i}, \alpha_{i} \in \mathbb{F}$ we have $\mathcal{M}_{m ; a, 1}=\sum_{i=1}^{d} \alpha_{i}\left(1, a_{i}\right)^{\otimes m}$, for every $0 \leq m \leq d$. Let $T \in \mathbb{F}^{d \times 2}$ be the matrix whose 1 st and 2 nd columns are $(1,1, \ldots, 1)^{\mathrm{T}}$ and $\left(a_{1}, a_{2}, \ldots, a_{d}\right)^{\mathrm{T}}$ respectively. Then $e_{d, i} T=\left(1, a_{i}\right)$. Define the symmetric matrix $B=\left(\beta_{i j}\right)=T T^{\mathrm{T}} \in \mathbb{F}^{d \times d}$. Now let $H$ be a weighted graph on $d$ vertices specified by $\left(\alpha_{1}, \ldots, \alpha_{d}\right) \in \mathbb{F}^{d}$ and $B=\left(\beta_{i j}\right) \in \mathbb{F}^{d \times d}$. Then for any degree- $d$ bounded graph $\mathrm{G}$ we have the following equality chain:

$$
\begin{aligned}
\operatorname{wm}_{a}(G) & =\operatorname{Holant}\left(G ;\left\{\mathcal{M}_{m ; a, 1}\right\}_{0 \leq m \leq d}\right)=\operatorname{Holant}\left(G^{\prime} ;\left\{\mathcal{M}_{m ; a, 1}\right\}_{0 \leq m \leq d} \mid\left(={ }_{2}\right)\right) \\
& =\operatorname{Holant}\left(G^{\prime} ;\left\{\sum_{i=1}^{d} \alpha_{i}\left(1, a_{i}\right)^{\otimes m}\right\}_{0 \leq m \leq d} \mid\left(=_{2}\right)\right)=\operatorname{Holant}\left(G^{\prime} ;\left\{\sum_{i=1}^{d} \alpha_{i}\left(e_{d, i} T\right)^{\otimes m}\right\}_{0 \leq m \leq d} \mid\left(=_{2}\right)\right) \\
& =\operatorname{Holant}\left(G^{\prime} ;\left\{\sum_{i=1}^{d} \alpha_{i} e_{d, i}^{\otimes m} T^{\otimes m}\right\}_{0 \leq m \leq d} \mid\left(={ }_{2}\right)\right)=\operatorname{Holant}\left(G^{\prime} ;\left\{\sum_{i=1}^{d} \alpha_{i} e_{d, i}^{\otimes m}\right\}_{0 \leq m \leq d} \mid T^{\otimes 2}(=2)\right) .
\end{aligned}
$$

where the last equation moving $T^{\otimes n}$ from the LHS of the Holant problem to $T^{\otimes 2}$ in the RHS, is called a holographic transformation [11,55] (the argument works for arbitrary fields). This follows from the associativity of the operation of tensor contraction. The EQUALITY function $(=2)$ is transformed to $T^{\otimes 2}(=2)$, which has the matrix form $T T^{\mathrm{T}}=B$. Hence this is precisely the function hom $(G, H)$. We have temporarily allowed 0 -weighted vertices; but in fact by the lower bound $q \geq d$ no 0 -weighted vertex exists, since otherwise by removing 0 -weighted vertices we would have $\operatorname{wm}_{a}(\cdot)=$ hom $\left(\cdot, H^{\prime}\right)$ with fewer vertices.

The inexpressibility with $|V(H)|=2$ for the exceptional case (8) holds even for simple graphs, by considering paths of 0,1 or 2 edges. Also, in this case it can be easily shown that $\mathrm{wm}_{a}=\mathrm{pm}$ is expressible as hom $(\cdot, H)$ where $|V(H)|=3$ : this can be done similarly to the expressibility proof above via a holographic transformation (then $H$ cannot have 0 -weighted vertices).

This proves Theorem 4.3.

Theorem 4.3. Let $\mathbb{F}$ be a field and $d \geq 2$. Then for the graph parameter \#WEIGHTED-MATCHING $a$ $\left(w_{a}\right)$ where $a \in \mathbb{F}$ as a function defined on degree-d bounded graphs the following hold:

1. $w m_{a}$ is not expressible as hom $(\cdot, H)$ with $|V(H)|<d$ even on degree-d bounded simple graphs.

2. If $\mathbb{F}$ is infinite, then $w_{a}$ is expressible as hom $(\cdot, H)$ with $|V(H)|=d$, with one exception (8) in which case the minimal value for $|V(H)|$ is 3 .

Note that \#PERfECT-MATCHING (pm) is just the special case $a=0$. Hence Theorem 4.3 also holds for $\mathrm{pm}$. 


\subsection{Proper edge $d$-colourings}

Next we show that the graph parameter \#d-EDGE-COLORING $\left(\mathrm{ec}_{d}\right)$ is not expressible as a GH function over any field of characteristic 0 . Given a graph $\mathrm{G}, \mathrm{ec}_{d}(G)$ counts the number of proper edge $d$-colourings in a graph, where $d \geq 1$ is the number of colours available.

Clearly, ec $c_{d}$ is a multiplicative graph parameter with ec $\left(K_{0}\right)=1$. Since char $\mathbb{F}=0, \mathbb{F}$ is infinite. Consider $K_{1}$ as a 1-labelled graph and the star graph $S_{d}$ with one internal node labelled by 1 and $d$ unlabelled external nodes all connected to node 1 , where $d \geq 1$. Consider the connection tensor $T\left(\mathrm{ec}_{d}, k, n\right)$ restricted to $\left\{K_{1}, S_{d}\right\}^{n}$. For $\left(G_{1}, \ldots, G_{n}\right) \in\left\{K_{1}, S_{d}\right\}^{n}$, if more than one $G_{i}=S_{d}$ then the product $G_{1} \cdots G_{n}$ has no proper edge $d$-colouring because the labelled vertex has degree $\geq 2 d>d$. Then it is easy to see that the connection tensor $T\left(\mathrm{ec}_{d}, k, n\right)$ restricted to $\left\{K_{1}, S_{d}\right\}^{n}$ has the form $\mathcal{M}_{1, d !}=[1, d !, 0, \ldots, 0] \in \operatorname{Sym}^{n}\left(\mathbb{F}^{2}\right)$, and $d ! \neq 0$ in $\mathbb{F}$ as char $\mathbb{F}=0$. Therefore by Lemma $4.1, \operatorname{rks}_{\mathrm{S}} \mathcal{M}_{1, d !} \geq n$ for $n \geq 1$. Hence $\operatorname{rks}_{\mathrm{S}} T\left(\mathrm{ec}_{d}, 1, n\right) \geq n$ for $n \geq 1$.

Now if ec $d$ were expressible as hom $(\cdot, H)$ for some weighted graph $H$ with $q=|V(H)|$, then by Theorem 3.1, $\mathrm{rks}_{\mathrm{S}} T\left(\mathrm{ec}_{d}, 1, n\right) \leq q$ for $n \geq 0$. This contradicts the upper bound when $n>q$. Hence $\mathrm{ec}_{d}$ is not expressible as a graph homomorphism function over any field $\mathbb{F}$ of char $\mathbb{F}=0$.

By the same remark for Theorem 4.1, the proof for Theorem 4.4 carries over to simple graphs.

Theorem 4.4. The graph parameter \#d-EDGE-COLORING $\left(e c_{d}\right)$ with $d \geq 1$ as a function defined on simple graphs is not expressible as a graph homomorphism function over any field of characteristic 0 .

\subsection{Vertex-disjoint cycle covers}

We show that the graph parameter vdcc (\#VERTEX-DISJOINT-CYCLE-COVER) which counts the number of vertex disjoint cycle covers in a graph is not expressible as a GH function over an arbitrary field. In a multigraph without loops a cycle is a vertex disjoint closed path of length at least 2. The graph parameter $\operatorname{vdcc}(G)=m \cdot 1 \in \mathbb{F}$, where $m$ is the number of edge subsets $E^{\prime}$ that form a vertex disjoint set of cycles that cover all vertices.

Clearly, vdcc is a multiplicative graph parameter with $\operatorname{vdcc}\left(K_{0}\right)=1$. Next, consider $K_{1}$ and $K_{3}$ as 1-labelled graphs. Note that $K_{3}$ is a cycle of 3 vertices. It is easy to see that the connection tensor $T(\operatorname{vdcc}, k, n)$ restricted to $\left\{K_{1}, K_{3}\right\}^{n}$ has the form $\mathcal{M}_{0,1}=[0,1,0, \ldots, 0] \in \operatorname{Sym}^{n}\left(\mathbb{F}^{2}\right)$ and therefore by Lemma $4.1, \operatorname{rks}_{S} \mathcal{M}_{0,1} \geq n$ for $n \geq 1$. Hence $\operatorname{rks} T$ (vdcc, $\left.1, n\right) \geq n$ for $n \geq 1$.

Now if vdcc were expressible as hom $(\cdot, H)$ for some weighted graph $H$ with $q=|V(H)|$, then by Theorem 3.1, $\operatorname{rks}_{\mathrm{S}} T(\mathrm{vdcc}, 1, n) \leq q$ for $n \geq 0$. This contradicts the upper bound when $n>q$. Hence vdcc is not expressible as a GH function over any field.

By the same remark for Theorem 4.1, the proof for Theorem 4.5 carries over to simple graphs.

Theorem 4.5. The graph parameter \#VERTEX-DISJOINT-CYCLE-COVER ( $v d c c$ ) as a function defined on simple graphs is not expressible as a graph homomorphism function over any field.

\subsection{Some previous work and model distinction}

Schrijver [46] gave a beautiful characterization of a graph property $G \mapsto f(G)$ to be expressible as $Z_{H}(\cdot)$ in (1) with complex $\beta_{i j}$ but all $\alpha_{i}=1$. However, there are some subtle differences in the definition. In particular, somewhat deviating from the standard definition (as in [27] and in this paper) the graphs $\mathrm{G}$ are allowed to have loops (indeed multiloops and multiedges) in [46]. The criterion is expressed in terms of a sum $\sum_{P \in \Pi_{V(G)}} \mu_{P} f(G / P)=0$ for all $G$ with $|V(G)|>f\left(K_{1}\right)$. Here $\Pi_{V(G)}$ is the partition lattice on $V(G), \mu_{P}$ is the Möbius inversion function on partitions, and the graph $G / P$ is obtained from $G$ by condensing all vertices in $P$ into one vertex. This condensation naturally creates loops. In [46] Schrijver carefully makes the distinction between the results in that paper with that of [27], and states that '[A]n interesting question is how these results relate'. 
In [48] Schrijver gave another characterization of expressibility as $Z_{H}(\cdot)$ in (1) with complex $\beta_{i j}$ but all $\alpha_{i}=1$. Again, the graphs $\mathrm{G}$ may have multiloops and multiedges. The criterion is expressed in terms of a rank bound of the $k$ th 'connection matrix' for every $k$. However, here the definition of the 'connection matrix' differs from that of [27]. They are defined over ' $k$-marked graphs' where the $k$ marked vertices are not necessarily distinct. This is in contrast to ' $k$-labeled graphs' in [27], as well as in this paper. Thus the $k$ th 'connection matrix' in [48] is a super matrix of the $k$ th 'connection matrix' in [27], and the rank bound is a stronger requirement.

At the end of this subsection we will show that this distinction is material, by showing that the well-known hardcore gas model in statistical physics, $\sum_{\text {ind } I \subseteq V(G)} \lambda^{|I|}$, where the sum is over all independent sets of $G$, cannot be expressed in the model discussed in $[46,48]$, i.e. without vertex weight. On the other hand, obviously the hardcore gas model is defined as a partition function of GH with vertex weight. In particular the rank bound for the 'connection matrix' in [48] must fail, while it must hold for that of [27], as well as for our connection tensor.

The terminology in the literature on this subject is unfortunately not uniform. In Lovász's book [39], separate from the partition function of GH as in (1), the model studied by Szegedy in [50] is called the 'edge coloring model'. This is essentially what we called Holant problems, or edge models. The slight difference is that Holant problems allow different constraint functions from a set assigned at different vertices, while the edge colouring model studied in [50] is a special case of Holant problems where for each arity $d$ a single symmetric vertex function $f_{d}$ is given and placed at all vertices of degree $d$.

In short, in edge models edges play the role of variables, and constraint functions are at vertices, and in vertex models vertices play the role of variables, and edges have binary constraint functions as well as vertices have unary constraint functions. Counting matchings, or perfect matchings, or valid edge colourings, or cycle covers, etc., are naturally expressible as edge models. It turns out that many orientation problems can also be expressed as edge models after a holographic transformation (by $Z=\frac{1}{\sqrt{2}}\left[\begin{array}{ll}1 & 1 \\ i & -i\end{array}\right]$ ). However, confusingly, the edge colouring model had also been called a vertex model in [22]. We also note that a preliminary version of [49] appeared as [47] which used the terminology 'vertex model', and that was changed to 'edge coloring model' in the final version [49]. In particular, these papers [22, 49, 50] do not address the expressibility of counting perfect matchings in the vertex model as in (1) with arbitrary vertex and edge weights, which is the subject of the present paper. However, Szegedy [51] proved that counting perfect matchings can be expressed as the limit of the partition function of a parametrized vertex model (see below).

Now we prove that the hardcore gas model fails the expressibility criterion of Schrijver [48] as a partition function of GH without vertex weight. We observe that if we take $A=\left[\begin{array}{ll}1 & 1 \\ 1 & 0\end{array}\right]$ indexed by $\{0,1\}$, which is the binary NAND function representing the independent set constraint, and the vertex weight $\alpha_{0}=1, \alpha_{1}=\lambda$, then $\sum_{\text {ind } I \subseteq V(G)} \lambda^{|I|}$ is clearly an instance of the expression (1). We now show that such an expression is impossible without vertex weight.

To state Schrijver's criterion we need a few definitions. Let $f$ be a graph property. A $k$-marked graph is a pair $(G, \mu)$ where $G=(V, E)$ is graph, and $\mu:[k] \rightarrow V$ is a function marking $k$ (not necessarily distinct) vertices. So a vertex may have several marks; this is the key distinction with $k$-labelled graphs. For $k$-marked graphs $\left(G_{1}, \mu\right)$ and $\left(G_{2}, v\right)$, the product $\left(G_{1}, \mu\right)\left(G_{2}, v\right)$ is the $k$ marked graph obtained by first taking a disjoint union and then merging all vertices of $G_{1}$ and $G_{2}$ that share a common mark. (Note that this merging process may produce multiedges and multiloops.) The $k$ th connection matrix $C_{k, f}$ in the sense of [48] is the infinite matrix whose rows and columns are indexed by all finite $k$-marked graphs and the entry at $\left(\left(G_{1}, \mu\right),\left(G_{2}, v\right)\right)$ is $f\left(\left(G_{1}, \mu\right)\left(G_{2}, v\right)\right)$. The expressibility criterion by Schrijver in [48] (Theorem 1 ) is that $f(\emptyset)=1$ and for some $c, \operatorname{rank}\left(C_{k, f}\right) \leq c^{k}$ for all $k$. 
We show that for the hardcore gas model this rank grows super exponentially. We consider the following submatrix. Let $\Pi_{k}$ be the set of all partitions of $[k]$. The rows and columns of the submatrix are indexed by the following $k$-marked graphs. For every $P=\left\{C_{1}, \ldots, C_{s}\right\} \in \Pi_{k}$ we have a $k$-marked graph $G_{P}$ with $s$ vertices denoted by $v_{1}, \ldots, v_{s}$, with no edges. For every $j \in[k]$, let $C_{t}$ be the unique set in $P$ that contains $j$, then we mark $v_{t}$ with $j$. Now suppose $P, Q \in \Pi_{k}$ are the indices of a row and column respectively, then the entry at $(P, Q)$ is the hardcore gas function evaluated at the product graph $G_{P} G_{Q}$. We observe that this product graph is just $G_{P \vee Q}$ where $P \vee Q$ is the least-upper-bound of $P$ and $Q$ in the lattice order of refinement: $P \leq P^{\prime}$ iff $P$ refines $P^{\prime}$. It follows that the entry at $(P, Q)$ is

$$
\prod_{D \in P \vee Q}(1+\lambda)=(1+\lambda)^{|P \vee Q|} .
$$

It is proved in [48] (Proposition 1) for $\lambda \neq-1,0,1,2, \ldots, k-2$, this matrix is nondegenerate. As its dimension $\left|\Pi_{k}\right|$ grows superexponentially in $k$, we see that the criterion in [48] is not satisfied. (However, it is easy to see that on $d$-regular graphs $(d \geq 1)$ the hardcore gas model is expressible as a partition function of $\mathrm{GH}$ without vertex weight, with the edge weight matrix $\left[\begin{array}{ll}1 & \lambda^{1 / d} \\ \lambda^{1 / d} & 0\end{array}\right]$, where $\lambda^{1 / d}$ can be taken to be any $d$ th root from $\lambda$. Basically, the entry 0 ensures that only independent sets can contribute non-zero weight to the partition function, and for each vertex assigned to be in an independent set each of the $d$ incident edges contributes a factor $\lambda^{1 / d}$.)

In contrast to Theorem 4.1, B. Szegedy in [51] shows that graph parameters arising from finite rank edge colouring models (defined in [51]) are in fact partition functions of singular vertex colouring models. Roughly speaking, for any fixed size (complete) graph $\mathrm{H}$ we consider vertex and edge weights of $H$ that depend on a parameter $t$, and denoting the weighted graph by $H_{t}$, we say a graph parameter is the partition function of a singular vertex colouring model if it is the limit of the graph homomorphism function hom $\left(G, H_{t}\right)$ when $t \rightarrow 0$. In particular, their connection matrices are exponentially rank bounded, a consequence by taking limits. The graph parameter \#PERFECTMATCHING (pm) is easily seen to be the partition function of a finite rank edge colouring model, so it can be expressed as a singular vertex colouring model. In fact, in [51] it is shown that $\mathrm{pm}(G)=$ $\lim _{t \rightarrow 0} \operatorname{hom}\left(G, H_{t}\right)$, where $H_{t}$ has the vertex weights $(1 / t,-1 / t)$, and edge weights $\left[\begin{array}{ll}1+t^{2} & 1 \\ 1 & 1\end{array}\right]$. Now we give a simple proof of this fact using holographic transformations.

We have the factorization $\left[\begin{array}{lll}1 & +t^{2} & 1 \\ 1 & 1\end{array}\right]=M M^{\mathrm{T}}$, where $M=\left[\begin{array}{ll}1 & t \\ 1 & 0\end{array}\right]$. A holographic transformation by $M$ transforms the vertex weight function of a vertex of degree $d$ to

$$
\frac{1}{t}\left[(1,0)^{\otimes d}-(0,1)^{\otimes d}\right] M^{\otimes d}=\frac{1}{t}\left[(1, t)^{\otimes d}-(1,0)^{\otimes d}\right]
$$

which is $\left[0,1, t, \ldots, t^{d-1}\right]$ in the symmetric signature notation, i.e. it takes values 0 and 1 on inputs of Hamming weight 0 and 1 , respectively, and $t^{i-1}$ on inputs of Hamming weight $i$, where $2 \leq i \leq$ $d$. Meanwhile, the edge weight function is transformed to the binary EQUALITY (represented by the identity matrix $I_{2}=\left[\begin{array}{ll}1 & 0 \\ 0 & 1\end{array}\right]$ ). Taking limit as $t \rightarrow 0$ we get the graph parameter pm.

\section{Proof of main theorem}

For now, we do not make any assumptions on the graph parameter $f$; we will introduce more assumptions as needed to prove the desired statements. When we speak of submonoids, subrings 
and subalgebras we require that the multiplicative identity coincide with that of the larger structure. When a subset with the induced operations forms a monoid, ring or algebra we will simply say that it is respectively a monoid, ring or algebra in the larger structure. We allow zero algebras and rings, in which $0=1$. Statements about such structures can be easily checked. A function of arity zero is a scalar. We identify a (labelled) graph with its (labelled) graph isomorphism class.

\subsection{The monoid and algebra of graphs}

For every finite set $S \subseteq \mathbb{Z}_{>0}$, we denote by $U_{S}$ the graph with $|S|$ nodes labelled by $S$ and no edges. Note that $U_{\emptyset}=K_{0}$ is the empty graph.

We put all $k$-labelled graphs into a single structure as follows. By a partially labelled graph we mean a finite graph in which some of the nodes are labelled by distinct positive integers. (All label sets are finite.) Two partially labelled graphs are isomorphic if there is an isomorphism between them preserving all labels. For two partially labelled graphs $G_{1}$ and $G_{2}$, let $G_{1} G_{2}$ denote the partially labelled graph obtained by taking the disjoint union of $G_{1}$ and $G_{2}$, and identifying the nodes with the same label; the union of the label sets becomes the labels of $G_{1} G_{2}$. This way we obtain a commutative monoid $\mathcal{P} \mathcal{L G}$ consisting of all isomorphism classes of finite partially labelled graphs with the empty graph $U_{\emptyset}$ being the identity. ${ }^{3}$ For every finite set $S \subseteq \mathbb{Z}_{>0}$, we call a partially labelled graph S-labelled, if its labels form the set $S$. We call a partially labelled graph $\subseteq S$-labelled, if its labels form a subset of $S$. We define $\mathcal{P} \mathcal{L G}(S)$ and $\mathcal{P} \mathcal{L} \mathcal{G}_{\subseteq}(S)$ to be the subsets of $\mathcal{P} \mathcal{L G}$ consisting of all isomorphism classes of $S$-labelled and $\subseteq S$-labelled graphs, respectively. Clearly $\mathcal{P} \mathcal{L} \mathcal{G}(S) \subseteq \mathcal{P} \mathcal{L} \mathcal{G}_{\subseteq}(S)$. Then both $\mathcal{P} \mathcal{L G}(S)$ and $\mathcal{P} \mathcal{L} \mathcal{G}_{\subseteq}(S)$ are commutative monoids in $\mathcal{P} \mathcal{L G}$. $\mathcal{P} \mathcal{L} \mathcal{G}_{\subseteq}(S)$ is a submonoid of $\mathcal{P} \mathcal{L G}$ with the same identity $U_{\emptyset}$, while $\mathcal{P} \mathcal{L G}(S)$ is a submonoid of $\mathcal{P} \mathcal{L G}$ iff $S=\emptyset$, as the identity in $\mathcal{P} \mathcal{L} \mathcal{G}(S)$ is $U_{S}$.

Let $\mathcal{G}$ denote the monoid algebra $\mathbb{F} \mathcal{P} \mathcal{L G}$ consisting of all finite formal linear combinations in $\mathcal{P} \mathcal{L} \mathcal{G}$ with coefficients from $\mathbb{F}$; they are called (partially labelled, $\mathbb{F}$-)quantum graphs. Restricting the labels to precisely $S$ or to subsets of $S$, we have $\mathbb{F} \mathcal{P} \mathcal{L G}(S)$ or $\mathbb{F} \mathcal{P} \mathcal{L} \mathcal{G}_{\subseteq}(S)$, the algebras of $S$ labelled or $\subseteq S$-labelled quantum graphs, denoted by $\mathcal{G}(S)$ or $\mathcal{G}_{\subseteq}(S)$, respectively. $\mathcal{G}(S)$ is an algebra inside $\mathcal{G}$ with $U_{S}$ being the multiplicative identity, and $\mathcal{G}_{\subseteq}(S)$ is a subalgebra of $\mathcal{G}$. The empty sum is the additive identity in all.

Because many definitions, notations and statements for $\mathcal{P} \mathcal{L G}(S), \mathcal{G}(S)$ and $\mathcal{P} \mathcal{L} \mathcal{G}_{\subseteq}(S), \mathcal{G}_{\subseteq}(S)$ appear similar, we will often commingle them to minimize repetitions, e.g. we use $\mathcal{G}_{(\subseteq)}(\bar{S})$ to denote either $\mathcal{G}(S)$ or $\mathcal{G}_{\subseteq}(S)$ (and the statements are asserted for both).

We can extend $f$ to a linear map on $\mathcal{G}$, and define an $n$-fold multilinear form, where $n \geq 1$,

$$
\left\langle x_{1}, \ldots, x_{n}\right\rangle_{(n)}=f\left(x_{1} \cdots x_{n}\right), \quad \text { for } x_{1}, \ldots, x_{n} \in \mathcal{G} .
$$

It is symmetric because $\mathcal{G}$ is commutative. Note that if we restrict each argument to $\mathcal{G}[k]$ and then write it with respect to the basis $\mathcal{P} \mathcal{L} G[k]$ of $\mathcal{G}[k]$, we get precisely the connection tensor (array) $T(f, k, n)$.

Let

$$
\mathcal{K}=\{x \in \mathcal{G} \mid \forall y \in \mathcal{G}, f(x y)=\langle x, y\rangle=0\}
$$

be the annihilator of $\mathcal{G}$. Clearly, $\mathcal{K}$ is an ideal in $\mathcal{G}$, so we can form the quotient algebra $\hat{\mathcal{G}}=\mathcal{G} / \mathcal{K}$ which is commutative as well. We denote its identity by $u_{\emptyset}=U_{\emptyset}+\mathcal{K}$. More generally, we let $u_{S}=U_{S}+\mathcal{K}$ for any finite subset $S \subseteq \mathbb{Z}_{>0}$. If $x \in \mathcal{K}$, then $f(x)=f\left(x U_{\emptyset}\right)=0$ and so $f$ can also be considered as a linear map on $\hat{\mathcal{G}}$ by $f(x+\mathcal{K})=f(x)$ for $x \in \mathcal{G}$. For a partially labelled graph $\mathrm{G}$ we denote by $\hat{G}=G+\mathcal{K}$ the corresponding element of $\hat{\mathcal{G}}$. More generally, we write $\hat{x}=x+\mathcal{K}$ for any

\footnotetext{
${ }^{3}$ In [27], the word semigroup instead of monoid is used. A monoid is a semigroup with identity, and all semigroups in [27] have or assume to have identity. Thus, our use of the term monoid is consistent with that of [27].
} 
$x \in \mathcal{G}$. Since $\mathcal{K}$ is an ideal in $\mathcal{G}$, the form $\langle\cdot, \ldots, \cdot\rangle_{(n)}$ on $\mathcal{G}$ induces an $n$-fold multilinear symmetric form on $\hat{\mathcal{G}}$, where $n \geq 1$,

$$
\left\langle x_{1}, \ldots, x_{n}\right\rangle_{(n)}=f\left(x_{1} \cdots x_{n}\right), \quad \text { for } x_{1}, \ldots, x_{n} \in \hat{\mathcal{G}} .
$$

We can also define

$$
\hat{\mathcal{G}}_{(\subseteq)}(S)=\left(\mathcal{G}_{(\subseteq)}(S)+\mathcal{K}\right) / \mathcal{K}=\left\{x+\mathcal{K} \mid x \in \mathcal{G}_{(\subseteq)}(S)+\mathcal{K}\right\}=\left\{x+\mathcal{K} \mid x \in \mathcal{G}_{(\subseteq)}(S)\right\} .
$$

It is easy to see that $\hat{\mathcal{G}}_{\subseteq}(S)$ is a subalgebra of $\hat{\mathcal{G}}$ with the same identity $u_{\emptyset}=U_{\emptyset}+\mathcal{K}$, and $\hat{\mathcal{G}}(S)$ is an algebra inside $\hat{\mathcal{G}}$ with the identity $u_{S}=U_{S}+\mathcal{K}{ }^{4}$

If $S, T \subseteq \mathbb{Z}_{>0}$ are finite subsets, then $\mathcal{P} \mathcal{L} \mathcal{G}_{(\subseteq)}(S) \cdot \mathcal{P} \mathcal{L} \mathcal{G}_{(\subseteq)}(T) \subseteq \mathcal{P} \mathcal{L} \mathcal{G}_{(\subseteq)}(S \cup T)$ so by linearity we get $\mathcal{G}_{(\subseteq)}(S) \mathcal{G}_{(\subseteq)}(T) \subseteq \mathcal{G}_{(\subseteq)}(S \cup T)$ and so, going to the quotients, we have $\hat{\mathcal{G}}_{(\subseteq)}(S) \hat{\mathcal{G}}_{(\subseteq)}(T) \subseteq$ $\hat{\mathcal{G}}_{(\subseteq)}(S \cup T)$. Also note that for a finite $S \subseteq \mathbb{Z}_{>0}$, we have $\mathcal{P} \mathcal{L G}(S) \subseteq \mathcal{P} \mathcal{L} \mathcal{G}_{\subseteq}(S)$ so by linearity $\mathcal{G}(S) \subseteq \mathcal{G}_{\subseteq}(S)$ and then by going to the quotients we obtain $\hat{\mathcal{G}}(S) \subseteq \hat{\mathcal{G}}_{\subseteq}(S)$.

Since $\overline{\mathcal{G}}_{(\subseteq)}(S) \cap \mathcal{K}$ is an ideal in $\mathcal{G}_{(\subseteq)}(S)$, we can also form another quotient algebra

$$
\tilde{\mathcal{G}}_{(\subseteq)}(S)=\mathcal{G}_{(\subseteq)}(S) /\left(\mathcal{G}_{(\subseteq)}(S) \mathcal{K}\right) \text {. }
$$

We have the following canonical isomorphisms between $\tilde{\mathcal{G}}_{(\subseteq)}(S)$ and $\hat{\mathcal{G}}_{(\subseteq)}(S)$.

Claim 1. Let $S \subseteq \mathbb{Z}_{>0}$ be finite. Then $\tilde{\mathcal{G}}_{(\subseteq)}(S) \cong \hat{\mathcal{G}}_{(\subseteq)}(S)$ as algebras via $x+\mathcal{G}_{(\subseteq)}(S) \cap \mathcal{K} \mapsto x+$ $\mathcal{K}, x \in \mathcal{G}_{(\subseteq)}(S)$. p. 8).

Proof. It follows from the Second Isomorphism Theorem for algebraic structures (see [19]

For finite $S \subseteq \mathbb{Z}_{>0}$, it is convenient to treat the algebras $\tilde{\mathcal{G}}_{(\subseteq)}(S)$ and $\hat{\mathcal{G}}_{(\subseteq)}(S)$ as separate objects despite this isomorphism in Claim 1 . As it will be seen later, the algebras $\tilde{\mathcal{G}}(S)$ with $S=[k]$, where $k \geq 0$, are naturally associated with the $k$ th connection tensors $T(f, k, n), n \geq 0$. Later we will need to work with various finite $S \subseteq \mathbb{Z}_{>0}$ simultaneously and need an ambient algebra in which dependencies between elements can be established. The algebras $\tilde{\mathcal{G}}(S)$ do not naturally possess this property as they are the quotients of the algebras $\mathcal{G}(S)$ which have no common element except 0 . However, the fact that $\hat{\mathcal{G}}(S) \subseteq \hat{\mathcal{G}}$ for any finite $S \subseteq \mathbb{Z}_{>0}$ will allow us to establish dependencies between their elements. In other words, $\hat{\mathcal{G}}$ will serve as the ambient algebra in which further derivations will take place. Next, the $\subseteq$-definitions will be needed to define a projection $\hat{\pi}_{S}: \mathcal{G} \rightarrow \mathcal{G}_{\subseteq}(S)$ (see Claims 6 and 7 ). This projection will be used later in the proof.

We say that elements $x, y \in \mathcal{G}$ (or $\hat{\mathcal{G}}$ ) are orthogonal (with respect to $f$ ), if $f(x y)=0$ and denote it by $x \perp y$. For a subset $A \subseteq \mathcal{G}$ (or $\hat{\mathcal{G}}$ ), denote by $A^{\perp}=\{x \in \mathcal{G}$ (or $\hat{\mathcal{G}}$ ) $\mid \forall y \in A, x \perp y\}$ the set of those elements in $\mathcal{G}$ (or $\hat{\mathcal{G}}$ ) orthogonal to all elements in $A$. Next, we say that subsets $A, B \subseteq \mathcal{G}$ (or $\hat{\mathcal{G}}$ ) are orthogonal (with respect to $f$ ), if $x \perp y$ for all $x \in A$ and $y \in B$. Similarly, we can talk about an element of $\mathcal{G}$ (or $\hat{\mathcal{G}}$ ) being orthogonal to a subset of $\mathcal{G}$ (or $\hat{\mathcal{G}}$ ) and vice versa. Note that the notion of orthogonality is symmetric since all the multiplication operations considered are commutative. From the definition, we have $\mathcal{K}=\mathcal{G}^{\perp}$. Next, denote (commingling the notations $\mathcal{K}_{S}$ and $\mathcal{K}_{\subseteq S}$ )

$$
\mathcal{K}_{(\subseteq) S}=\left\{x \in \mathcal{G}_{(\subseteq)}(S) \mid \forall y \in \mathcal{G}_{(\subseteq)}(S), x \perp y\right\}=\mathcal{G}_{(\subseteq)}(S) \cap\left(\mathcal{G}_{(\subseteq)}(S)\right)^{\perp} .
$$

Clearly, $\mathcal{K}_{(\subseteq) S}$ is an ideal in $\mathcal{G}_{(\subseteq)}(S)$, so we can form yet another quotient algebra $\mathcal{G}_{(\subseteq)}(S) / \mathcal{K}_{(\subseteq) S}$.

Next, we define an orthogonal projection from $\hat{\mathcal{G}}$ to the subalgebra $\hat{\mathcal{G}} \subseteq(S)$. We will show how to do it in a series of lemmas. Let $S \subseteq \mathbb{Z}_{>0}$ be finite. For every partial labelled graph $G$, let $G_{S}$ denote

\footnotetext{
${ }^{4}$ In contrast to [27] we cannot in general normalize $f$ to make all elements $u_{S}$ the same in the quotient algebra $\hat{\mathcal{G}}$, for various finite $S \subseteq \mathbb{Z}_{>0}$. This is because in our more general setting, it is possible $f\left(K_{1}\right)=0$, in which case the normalization step from [27] fails. For graph parameters expressible as a graph homomorphism function, this corresponds to the case when all vertex weights sum to 0 .
} 
the $\subseteq S$-labelled graph obtained by deleting the labels not in $S$ from the vertices of $G$ (unlabeling such vertices). Extending this map by linearity, we get a linear map $\pi_{S}: \mathcal{G} \rightarrow \mathcal{G}_{\subseteq}(S)$. Note that $\left(\pi_{S}\right)_{\mid \mathcal{G}_{\subseteq}(S)}=\operatorname{id}_{\mid \mathcal{G}_{\subseteq}(S)}$. In particular, $\pi_{S}: \mathcal{G} \rightarrow \mathcal{G}_{\subseteq}(S)$ is surjective.

Claim 2. Let $S \subseteq \mathbb{Z}_{>0}$ be finite. If $x \in \mathcal{G}$ and $y \in \mathcal{G}_{\subseteq}(S)$, then

$$
f(x y)=f\left(\pi_{S}(x) y\right) .
$$

Proof. For every $G_{1} \in \mathcal{P} \mathcal{L G}$ and $G_{2} \in \mathcal{P} \mathcal{L} \mathcal{G}_{\subseteq}(S)$, the graphs $G_{1} G_{2}$ and $\pi_{S}\left(G_{1}\right) G_{2}$ are isomorphic as unlabelled graphs. Hence $f\left(G_{1} G_{2}\right)=f\left(\pi_{S}\left(G_{1}\right) G_{2}\right)$ as $f$ ignores labels. The claim follows by linearity.

Claim 3. Let $S \subseteq \mathbb{Z}_{>0}$ be finite. If $x \in \mathcal{G}$, then $x-\pi_{S}(x) \in\left(\mathcal{G}_{\subseteq}(S)\right)^{\perp}$.

Proof. Fix any $y \in \mathcal{G}_{\subseteq}(S)$. By Claim 5.1, $f(x y)=f\left(\pi_{S}(x) y\right)$ so $f\left(\left(x-\pi_{S}(x)\right) y\right)=0$. Thus $x-$ $\pi_{S}(x) \in\left(\mathcal{G}_{\subseteq}(S)\right)^{\perp}$.

So for any $x \in \mathcal{G}$, we can write $x=\pi_{S}(x)+\left(x-\pi_{S}(x)\right)$ where $\pi_{S}(x) \in \mathcal{G}_{\subseteq}(S)$, and $x-\pi_{S}(x) \in$ $\left(\mathcal{G}_{\subseteq}(S)\right)^{\perp}$. This gives a decomposition $\mathcal{G}=\mathcal{G}_{\subseteq}(S)+\left(\mathcal{G}_{\subseteq}(S)\right)^{\perp}$. To get a direct sum decomposition, we need to pass to the quotient algebra. But to do so properly we need some more properties.

Claim 4. Let $S \subseteq \mathbb{Z}_{>0}$ be finite. Then $\mathcal{K}_{S}=\mathcal{G}(S) \cap \mathcal{K}$.

Proof. Clearly, $\mathcal{G}(S) \cap \mathcal{K}=\mathcal{G}(S) \cap \mathcal{G}^{\perp} \subseteq \mathcal{G}(S) \cap(\mathcal{G}(S))^{\perp}=\mathcal{K}_{S}$, so we only need to prove the reverse inclusion. Let $x \in \mathcal{K}_{S}=\mathcal{G}(S) \cap(\mathcal{G}(S))^{\perp}$. Take any $y \in \mathcal{G}$. Then

$$
f(x y) \stackrel{(1)}{=} f\left(x \pi_{S}(y)\right) \stackrel{(2)}{=} f\left(x U_{S} \pi_{S}(y)\right) \stackrel{(3)}{=} 0 .
$$

Here step (1) uses Claim 2 as $x \in \mathcal{G}(S) \subseteq \mathcal{G}_{\subseteq}(S)$; (2) is true because $x=x U_{S}$ for $x \in \mathcal{G}(S)$; (3) is true as $\pi_{S}(y) \in \mathcal{G}_{\subseteq}(S)$ so $U_{S} \pi_{S}(y) \in \mathcal{G}(S)$, and as $x \in(\mathcal{G}(S))^{\perp}$. Then $x \in \mathcal{K}$ so $x \in \mathcal{G}(S) \cap \mathcal{K}$, implying $\mathcal{K}_{S} \subseteq \mathcal{G}(S) \cap \mathcal{K}$.

Claim 5. Let $S \subseteq \mathbb{Z}_{>0}$ be finite. Then $\mathcal{K}_{\subseteq S}=\mathcal{G}_{\subseteq}(S) \cap \mathcal{K}$.

Proof. Clearly, $\mathcal{G}_{\subseteq}(S) \cap \mathcal{K}=\mathcal{G}_{\subseteq}(S) \cap \mathcal{G}^{\perp} \subseteq \mathcal{G}_{\subseteq}(S) \cap\left(\mathcal{G}_{\subseteq}(S)\right)^{\perp}=\mathcal{K}_{\subseteq S}$, so we only need to prove the reverse inclusion. Let $x \in \mathcal{K}_{\subseteq S}=\mathcal{G}_{\subseteq}(S) \cap\left(\mathcal{G}_{\subseteq}(S)\right)^{\perp}$. Take any $y \in \overline{\mathcal{G}}$. Then

$$
f(x y) \stackrel{(1)}{=} f\left(x \pi_{S}(y)\right) \stackrel{(2)}{=} 0 .
$$

Here step (1) uses Claim 2; (2) is true as $\pi_{S}(y) \in \mathcal{G}_{\subseteq}(S)$ and $x \in\left(\mathcal{G}_{\subseteq}(S)\right)^{\perp}$. Then $x \in \mathcal{K}$ so $x \in$ $\mathcal{G}_{\subseteq}(S) \cap \mathcal{K}$, implying $\mathcal{K}_{\subseteq S} \subseteq \mathcal{G}_{\subseteq}(S) \cap \mathcal{K}$.

It follows from Claims 4 and 5 that $\mathcal{G}_{(\subseteq)}(S) / \mathcal{K}_{(\subseteq) S}=\mathcal{G}_{(\subseteq)}(S) /\left(\mathcal{G}_{(\subseteq)}(S) \cap \mathcal{K}\right)=\tilde{\mathcal{G}}_{(\subseteq)}(S)$ so the (canonical) isomorphism of algebras from Claim 1 takes the following form:

$$
\tilde{\mathcal{G}}_{(\subseteq)}(S) \cong \hat{\mathcal{G}}_{(\subseteq)}(S), \quad x+\mathcal{K}_{(\subseteq) S} \mapsto x+\mathcal{K}, \quad x \in \mathcal{G}_{(\subseteq)}(S) .
$$

Claim 6. Let $S \subseteq \mathbb{Z}_{>0}$ be finite. For the linear map $\pi_{S}: \mathcal{G} \rightarrow \mathcal{G}_{\subseteq}(S)$ we have $\pi_{S}(\mathcal{K})=\mathcal{K}_{\subseteq S}$.

Proof. Because $\left(\pi_{S}\right)_{\mid \mathcal{G}_{\subseteq}(S)}=\operatorname{id}_{\mid \mathcal{G}_{\subseteq}(S)}$ and, by Lemma $5, \mathcal{K}_{\subseteq S}=\mathcal{G}_{\subseteq}(S) \cap \mathcal{K}$, we infer that $\pi_{S}(\mathcal{K}) \supseteq$ $\mathcal{K}_{\subseteq S}$. For the reverse inclusion, let $x \in \mathcal{K}$. Fix any $y \in \mathcal{G}_{\subseteq}(S)$. Then by Claim $2, f\left(\pi_{S}(x) y\right)=f(x y)=$ 0 , the last equality is true because $x \in \mathcal{K}$. Hence $\pi_{S}(x) \in \mathcal{K}_{\subseteq S}$ so that $\pi_{S}(\mathcal{K}) \subseteq \mathcal{K}_{\subseteq S}$.

For the linear map $\pi_{S}: \mathcal{G} \rightarrow \mathcal{G}_{\subseteq}(S) \subseteq \mathcal{G}$ by Lemmas 6 and $5, \pi_{S}(\mathcal{K})=\mathcal{K}_{\subseteq S}=\mathcal{G}_{\subseteq}(S) \cap \mathcal{K}$, so that we have the well-defined linear map (which we denote by $\hat{\pi}_{S}$ )

$$
\hat{\pi}_{S}: \hat{\mathcal{G}} \rightarrow \hat{\mathcal{G}}_{\subseteq}(S), \quad \hat{\pi}_{S}(x+\mathcal{K})=\pi_{S}(x)+\mathcal{K}, \quad x \in \mathcal{G} .
$$

It is easy to see that $\left(\hat{\pi}_{S}\right)_{\mid \hat{\mathcal{G}}_{\subseteq}(S)}=\mathrm{id}_{\mid \hat{\mathcal{G}}_{\subseteq}(S)}$. In particular, $\hat{\pi}_{S}: \hat{\mathcal{G}} \rightarrow \hat{\mathcal{G}} \subseteq_{\subseteq}(S)$ is surjective. 
Claim 7. Let $S \subseteq \mathbb{Z}_{>0}$ be finite. Then $\hat{\mathcal{G}}=\hat{\mathcal{G}}_{\subseteq}(S) \oplus\left(\hat{\mathcal{G}}_{\subseteq}(S)\right)^{\perp}$ via $x=\hat{\pi}_{S}(x)+\left(x-\hat{\pi}_{S}(x)\right), x \in \hat{\mathcal{G}}$.

Proof. First, let $x \in \hat{\mathcal{G}}$. Write $x=y+\mathcal{K}$ where $y \in \mathcal{G}$. Then $\hat{\pi}_{S}(x)=\pi_{S}(y)+\mathcal{K}$ and $\pi_{S}(y) \in \mathcal{G}_{\subseteq}(S)$. We have $x-\hat{\pi}_{S}(x)=y-\pi_{S}(y)+\mathcal{K}$. By Claim $5.1, y-\pi_{S}(y) \in\left(\mathcal{G}_{\subseteq}(S)\right)^{\perp}$ so that $x-\hat{\pi}_{S}(x) \in$ $\left(\hat{\mathcal{G}}_{\subseteq}(S)\right)^{\perp}$, since the bilinear form on $\mathcal{G}$ extends to $\hat{\mathcal{G}}$ in $(11)$.

So we only need to show that $\hat{\mathcal{G}} \subseteq_{\subseteq}(S) \cap\left(\hat{\mathcal{G}} \subseteq_{\subseteq}(S)\right)^{\perp}=0(=\{\mathcal{K}\})$. Let $z$ belong to this intersection. Write $z=t+\mathcal{K}=t^{\prime}+\mathcal{K}$ where $t \in \mathcal{G}_{\subseteq}(S)$ and $t^{\prime} \in\left(\mathcal{G}_{\subseteq}(S)\right)^{\perp}$. Then clearly $t-t^{\prime} \in \mathcal{K} \subseteq\left(\mathcal{G}_{\subseteq}(S)\right)^{\perp}$, and so $t=\left(t-t^{\prime}\right)+t^{\prime} \in\left(\mathcal{G}_{\subseteq}(S)\right)^{\perp}$. Thus $t \in \mathcal{G}_{\subseteq}(S) \cap\left(\mathcal{G}_{\subseteq}(S)\right)^{\perp}=\mathcal{K}_{\subseteq S} \subseteq \mathcal{K}$, the last inclusion holds by Claim 5. Therefore $z=t+\mathcal{K}=\mathcal{K}$, implying that $\hat{\mathcal{G}}_{\subseteq}(S) \cap\left(\hat{\mathcal{G}}_{\subseteq}(S)\right)^{\perp}=0$.

Thus Claim 7 allows us to rightfully call $\hat{\pi}_{S}: \hat{\mathcal{G}} \rightarrow \hat{\mathcal{G}} \subseteq_{\subseteq}(S)$ an orthogonal projection of $\hat{\mathcal{G}}$ to $\hat{\mathcal{G}}_{\subseteq}(S)$.

If $S, T \subseteq \mathbb{Z}_{>0}$ are finite subsets, then $\pi_{S}\left(\mathcal{P} \mathcal{L} \mathcal{G}_{(\subseteq)}(T)\right)=\mathcal{P} \mathcal{L} \mathcal{G}_{(\subseteq)}(S \cap T)$, where the projection is surjective because the restriction $\left(\pi_{S}\right)_{\mid \mathcal{P} \mathcal{L} \mathcal{G}_{(\subseteq)}(S \cap T)}=\mathrm{id}_{\mid \mathcal{P} \mathcal{L} \mathcal{G}_{(\subseteq)}(S \cap T)}$. So by linearity we get $\pi_{S}\left(\mathcal{G}_{(\subseteq)}(T)\right)=\mathcal{G}_{(\subseteq)}(S \cap T)$. Going to the quotients, we conclude that $\hat{\pi}_{S}\left(\hat{\mathcal{G}}_{(\subseteq)}(T)\right)=\hat{\mathcal{G}}_{(\subseteq)}(S \cap T)$.

Claim 8. Let $n \geq 2$ and $S \subseteq \mathbb{Z}_{>0}$ be finite. Then for any $x \in \mathcal{G}_{(\subseteq)}(S)$, we have $x \in \mathcal{K}_{(\subseteq) S}$ iff $f\left(x x_{1} \cdots x_{n-1}\right)=0$ for all $x_{1}, \ldots, x_{n-1} \in \mathcal{G}_{(\subseteq)}(S)$.

Proof. For $\Rightarrow$, it suffices to note that $\mathcal{G}_{(\subseteq)}(S)$ is closed under multiplication (in $\mathcal{G}$ ). To prove $\Leftarrow$, note that $n-2 \geq 0$ and for any $y \in \mathcal{G}_{(\subseteq)}(S)$, we have $f(x y)=f\left(x y U^{n-2}\right)=0$, where $U=U_{\emptyset}$ in the $\mathcal{G}_{\subseteq}(S)$ case and $U=U_{S}$ in the $\mathcal{G}(S)$ case, so $x \in \mathcal{K}_{(\subseteq) S}$.

The primary goal of the various Claims above is to define the projection $\hat{\pi}_{S}: \mathcal{G} \rightarrow \mathcal{G}_{\subseteq}(S)$ to be used later and to prove Lemmas 5.1 and 5.2.

Lemma 5.1. Let $S \subseteq \mathbb{Z}_{>0}$ be finite. Then the annihilator of $\hat{\mathcal{G}}_{(\subseteq)}(S)$ in $\hat{\mathcal{G}}_{(\subseteq)}(S)$ is zero, i.e. if $x \in$ $\hat{\mathcal{G}}_{(\subseteq)}(S)$ and $f(x y)=0$ for every $y \in \hat{\mathcal{G}}_{(\subseteq)}(S)$, then $x$ is the zero element of $\hat{\mathcal{G}}_{(\subseteq)}(S)$, namely $\mathcal{K}$.

Proof. Let $x \in \hat{\mathcal{G}}_{(\subseteq)}(S)$ be an element satisfying the hypothesis of the lemma. Write $x=h_{1}+\mathcal{K}$ where $h_{1} \in \mathcal{G}_{(\subseteq)}(S)$. By hypothesis, for every $y \in \hat{\mathcal{G}}_{(\subseteq)}(S)$ we have $f(x y)=0$. Let $h_{2} \in \mathcal{G}_{(\subseteq)}(S)$ and put $y=h_{2}+\mathcal{K} \in \hat{\mathcal{G}}_{(\subseteq)}(S)$. Then $x y=h_{1} h_{2}+\mathcal{K}$. By the definition of $f$ on $\hat{\mathcal{G}}, f\left(h_{1} h_{2}\right)=f(x y)=0$. Hence $h_{1} \in \mathcal{K}_{(\subseteq) S} \subseteq \mathcal{K}$ where the last inclusion is true by Claims 4 and 5 . This implies that $x$ is the zero element of $\hat{\mathcal{G}}_{(\subseteq)}(S)$, which is $\mathcal{K}$.

Lemma 5.2. Let $k, r \geq 0$ and $n \geq \max (2, r)$. Suppose the connection tensor $T(f, k, n)$ can be expressed as

$$
T(f, k, n)=\sum_{i=1}^{r} a_{i} x_{i}^{\otimes n},
$$

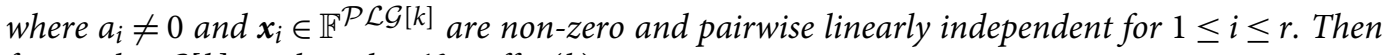
for any $h \in \mathcal{G}[k]$, we have $h \in \mathcal{K}_{[k]}$ iff $\boldsymbol{x}_{i}(h)=0,1 \leq i \leq r$.

Proof. The lemma is clearly true for $r=0$. Let $r \geq 1$. By Claim $8, h \in \mathcal{K}_{[k]}$ iff $f\left(h h_{1} \cdots h_{n-1}\right)=0$ for all $h_{1}, \ldots, h_{n-1} \in \mathcal{G}[k]$. In terms of $T(f, k, n)$, this is equivalent to $(T(f, k, n))(h, \cdot, \ldots, \cdot)=0$ which is the same as

$$
\sum_{i=1}^{r} a_{i} \mathbf{x}_{i}(h) \mathbf{x}_{i}^{\otimes(n-1)}=0 .
$$

Now if $\mathbf{x}_{i}(h)=0,1 \leq i \leq r$, then this equality clearly holds. Conversely, if this equality holds, then by Lemma A.5, $a_{i} \mathbf{x}_{i}(h)=0$ but $a_{i} \neq 0$ so $\mathbf{x}_{i}(h)=0,1 \leq i \leq r$.

We will also need the following lemma that classifies all subalgebras of $\mathbb{F}^{m}$ for $m \geq 0$. A proof is given in Subsection A.3. Recall that we allow zero algebras and require any subalgebra of an algebra to share the multiplicative identity. 
Lemma 5.3. All subalgebras of $\mathbb{F}^{m}$, where $m \geq 0$, are of the following form: For some partition $[m]=\bigsqcup_{i=1}^{s} \mathcal{I}_{i}$, where $s \geq 0$, and $\mathcal{I}_{i} \neq \emptyset$ for $i \in[s]$, the subalgebra has equal values on each $I_{i}$,

$$
\mathbb{F}^{\left(\mathcal{I}_{1}, \ldots, \mathcal{I}_{s}\right)}=\left\{\left(c_{1}, \ldots, c_{m}\right) \in \mathbb{F}^{m} \mid \forall i \in[s], \forall j, j^{\prime} \in \mathcal{I}_{i}, c_{j}=c_{j^{\prime}}\right\}
$$

\subsection{Building an algebra isomorphism}

In this part of the proof regarding $f$, for an arbitrary fixed $k \geq 0$, we assume that there exists $n=n_{k} \geq 2$ such that $\operatorname{rks}_{S} T(f, k, n) \leq n-1$. We will pick an arbitrary such $n$ and call it $n_{k}$, and then write $r_{k}=\operatorname{rks}_{S} T\left(f, k, n_{k}\right)$. (Note that this is weaker than the uniform exponential boundedness in $k$ for $\operatorname{rk}_{\mathrm{S}} T(f, k, n)$ in Theorem 3.2, nor do we require $f\left(K_{0}\right)=1$ here.)

Then, for $n=n_{k}$, we can write

$$
T(f, k, n)=\sum_{i=1}^{r_{k}} a_{k, n, i} \mathbf{x}_{k, n, i}^{\otimes n} .
$$

Then $a_{k, n, i} \neq 0$ and $0 \neq \mathbf{x}_{k, n, i} \in \mathbb{F}^{\mathcal{P} \mathcal{L} \mathcal{G}[k]}$ are pairwise linearly independent for $1 \leq i \leq r_{k}$.

Define the linear map

$$
\Phi_{k, n}: \mathcal{G}[k] \rightarrow \mathbb{F}^{r_{k}}, \quad \Phi_{k, n}(h)=\left(\mathbf{x}_{k, n, i}(h)\right)_{i=1, \ldots, r_{k}}, \quad h \in \mathcal{G}[k] .
$$

We show that $\Phi_{k, n}: \mathcal{G}[k] \rightarrow \mathbb{F}^{r_{k}}$ is a surjective algebra homomorphism, after a normalization step (to be carried out later). Clearly, as $n \geq 2$,

$$
h_{1} \cdot h_{2} \cdot h_{3} \cdots h_{n}=\left(h_{1} h_{2}\right) \cdot U_{k} \cdot h_{3} \cdots h_{n}
$$

so

$$
f\left(h_{1} \cdot h_{2} \cdot h_{3} \cdots h_{n}\right)=f\left(\left(h_{1} h_{2}\right) \cdot U_{k} \cdot h_{3} \cdots h_{n}\right)
$$

for all $h_{1}, \ldots, h_{n} \in \mathcal{G}[k]$. (When $n=2$ this is $f\left(h_{1} h_{2}\right)=f\left(\left(h_{1} h_{2}\right) U_{k}\right)$.) Therefore

$$
(T(f, k, n))\left(h_{1}, h_{2}, \cdot, \ldots, \cdot\right)=(T(f, k, n))\left(h_{1} h_{2}, U_{k}, \cdot, \ldots, \cdot\right)
$$

for all $h_{1}, h_{2} \in \mathcal{G}[k]$. In terms of the decomposition in (14), this is equivalent to

$$
\sum_{i=1}^{r_{k}} a_{k, n, i} \mathbf{x}_{k, n, i}\left(h_{1}\right) \mathbf{x}_{k, n, i}\left(h_{2}\right) \mathbf{x}_{k, n, i}^{\otimes(n-2)}=\sum_{i=1}^{r_{k}} a_{k, n, i} \mathbf{x}_{k, n, i}\left(h_{1} h_{2}\right) \mathbf{x}_{k, n, i}\left(U_{k}\right) \mathbf{x}_{k, n, i}^{\otimes(n-2)} .
$$

It follows that

$$
\sum_{i=1}^{r_{k}} a_{k, n, i}\left(\mathbf{x}_{k, n, i}\left(h_{1}\right) \mathbf{x}_{k, n, i}\left(h_{2}\right)-\mathbf{x}_{k, n, i}\left(h_{1} h_{2}\right) \mathbf{x}_{k, n, i}\left(U_{k}\right)\right) \mathbf{x}_{k, i}^{\otimes(n-2)}=0
$$

for any $h_{1}, h_{2} \in \mathcal{G}[k]$. The condition $r_{k} \leq n-1$ allows us to apply Lemma A.5. Since $a_{k, n, i} \neq 0$ for $1 \leq i \leq r_{k}$, we obtain that

$$
\mathbf{x}_{k, n, i}\left(h_{1}\right) \mathbf{x}_{k, n, i}\left(h_{2}\right)=\mathbf{x}_{k, n, i}\left(h_{1} h_{2}\right) \mathbf{x}_{k, n, i}\left(U_{k}\right), \quad h_{1}, h_{2} \in \mathcal{G}[k], \quad 1 \leq i \leq r_{k} .
$$

Let $1 \leq i \leq r_{k}$. Since $\mathbf{x}_{k, n, i} \neq 0$, there exists $h \in \mathcal{G}[k]$ such that $\mathbf{x}_{k, n, i}(h) \neq 0$. Substituting $h_{1}=h_{2}=h$ into (16), we infer that $\mathbf{x}_{k, n, i}\left(U_{k}\right) \neq 0,1 \leq i \leq r_{k}$.

Therefore we can assume in (14) that each $\mathbf{x}_{k, n, i}$ is normalized so that $\mathbf{x}_{k, n, i}\left(U_{k}\right)=1\left(1 \leq i \leq r_{k}\right)$. Combined with this, condition (16) becomes for $1 \leq i \leq r_{k}$,

$$
\begin{cases}\mathbf{x}_{k, n, i}\left(h_{1} h_{2}\right) & =\mathbf{x}_{k, n, i}\left(h_{1}\right) \mathbf{x}_{k, n, i}\left(h_{2}\right), \quad h_{1}, h_{2} \in \mathcal{G}[k] \\ \mathbf{x}_{k, n, i}\left(U_{k}\right) & =1\end{cases}
$$


so the linear functions $\mathbf{x}_{k, n, i}: \mathcal{G}[k] \rightarrow \mathbb{F}, 1 \leq i \leq r_{k}$ are algebra homomorphisms. Then we have

$$
\begin{gathered}
\Phi_{k, n}(g h)=\left(\mathbf{x}_{k, n, 1}(g h), \ldots, \mathbf{x}_{k, n, r_{k}}(g h)\right)=\left(\mathbf{x}_{k, n, 1}(g) \mathbf{x}_{k, n, 1}(h), \ldots, \mathbf{x}_{k, n, r_{k}}(g) \mathbf{x}_{k, n, r_{k}}(h)\right) \\
=\left(\mathbf{x}_{k, n, 1}(g), \ldots, \mathbf{x}_{k, n, r_{k}}(g)\right) \cdot\left(\mathbf{x}_{k, n, 1}(h), \ldots, \mathbf{x}_{k, n, r_{k}}(h)\right)=\Phi_{k, n}(g) \Phi_{k, n}(h) .
\end{gathered}
$$

So we have

$$
\begin{gathered}
\Phi_{k, n}(g h)=\Phi_{k, n}(g) \Phi_{k, n}(h), \quad g, h \in \mathcal{G}[k], \\
\Phi_{k, n}\left(U_{k}\right)=\left(\mathbf{x}_{k, n, 1}\left(U_{k}\right) \ldots, \mathbf{x}_{k, n, r_{k}}\left(U_{k}\right)\right)=\underbrace{(1, \ldots, 1)}_{r_{k} \text { times }} \in \mathbb{F}^{r_{k}},
\end{gathered}
$$

and therefore $\Phi_{k, n}: \mathcal{G}[k] \rightarrow \mathbb{F}^{r}$ is an algebra homomorphism. We now prove its surjectivity. Clearly, $\operatorname{im}\left(\Phi_{k, n}\right)$ is a subalgebra of $\mathbb{F}^{r_{k}}$. By Lemma 5.3, we may assume that $\operatorname{im}\left(\Phi_{k, n}\right)$ has the form $\mathbb{F}^{\left(\mathcal{I}_{1}, \ldots, \mathcal{I}_{s}\right)}$ for some partition $\left\{\mathcal{I}_{1}, \ldots, \mathcal{I}_{s}\right\}$ of $\left[r_{k}\right]$. The pairwise linear independence of $\mathbf{x}_{k, n, i}$ for $1 \leq i \leq r_{k}$ implies that for any $1 \leq i_{1}<i_{2} \leq r_{k}$, we have $\mathbf{x}_{k, n, i_{1}} \neq \mathbf{x}_{k, n, i_{2}}$, so there exists $h \in \mathcal{G}[k]$ such that $\mathbf{x}_{k, n, i_{1}}(h) \neq \mathbf{x}_{k, n, i_{2}}(h)$. Since each $\mathcal{I}_{i} \neq \emptyset$, it follows that $\left|\mathcal{I}_{i}\right|=1$ for $1 \leq i \leq s$. Hence $\operatorname{im}\left(\Phi_{k, n}\right)=\mathbb{F}^{\left(\{1\}, \ldots,\left\{r_{k}\right\}\right)}=\mathbb{F}^{r_{k}}$. We have shown that $\Phi_{k, n}: \mathcal{G}[k] \rightarrow \mathbb{F}^{r_{k}}$ is surjective.

This results in the following lemma.

Lemma 5.4. Let $k \geq 0$. The constructed map $\Phi_{k, n}: \mathcal{G}[k] \rightarrow \mathbb{F}^{r_{k}}$ defined in (15) is a surjective algebra homomorphism, after the normalization to set $\boldsymbol{x}_{k, n, i}\left(U_{k}\right)=1$ for $1 \leq i \leq r_{k}$.

Next, by $r_{k} \leq n-1$ and $n \geq 2$, clearly $n \geq \max \left(2, r_{k}\right)$, so Lemma 5.2 applies. So we have

$$
\operatorname{ker} \Phi_{k, n}=\left\{h \in \mathcal{G}[k] \mid \mathbf{x}_{k, n, i}(h)=0,1 \leq i \leq r_{k}\right\}=\mathcal{K}_{[k]},
$$

where the first equality is by the definition of $\Phi_{k, n}$, and the second equality is by Lemma 5.2. Note that by Claim 4, we have $\mathcal{K}_{[k]}=\mathcal{G}[k] \cap \mathcal{K}$. Then $\Phi_{k, n}: \mathcal{G}[k] \rightarrow \mathbb{F}^{r}$ factors through $\mathcal{G}[k] / \operatorname{ker} \Phi_{k, n}=\mathcal{G}[k] / \mathcal{K}_{[k]}=\tilde{\mathcal{G}}[k]$, inducing an algebra isomorphism

$$
\tilde{\Phi}_{k, n}: \tilde{\mathcal{G}}[k] \rightarrow \mathbb{F}^{r_{k}}, \quad \tilde{\Phi}_{k, n}\left(h+\mathcal{K}_{[k]}\right)=\left(\mathbf{x}_{k, n, 1}(h), \ldots, \mathbf{x}_{k, n, r_{k}}(h)\right), \quad h \in \mathcal{G}[k] .
$$

It follows that $\operatorname{dim} \tilde{\mathcal{G}}[k]=\operatorname{dim} \mathbb{F}^{r_{k}}=r_{k}$. In particular, $\tilde{\mathcal{G}}[k]$ is a finite dimensional algebra. Applying Lemma A.3, we get $\operatorname{dim} \tilde{\mathcal{G}}[k]=\operatorname{dim} \operatorname{span}\left\{\mathbf{x}_{k, n, i}\right\}_{i=1}^{r_{k}}$. Then $\operatorname{dim} \operatorname{span}\left\{\mathbf{x}_{k, n, i}\right\}_{i=1}^{r_{k}}=r_{k}$ implying that $\mathbf{x}_{k, n, i}, 1 \leq i \leq r_{k}$, are linearly independent. (Note that we started off only assuming they are non-zero and pairwise linearly independent.) We formalize some of the results obtained above.

Lemma 5.5. Let $k \geq 0$. Assume there exists $n=n_{k} \geq 2$ such that $r_{k}=r k_{S} T\left(f, k, n_{k}\right) \leq n_{k}-1$. Then the constructed map

$$
\tilde{\Phi}_{k, n}: \tilde{\mathcal{G}}[k] \rightarrow \mathbb{F}^{r_{k}}, \quad \tilde{\Phi}_{k}\left(h+\mathcal{K}_{[k]}\right)=\left(x_{k, n, 1}(h), \ldots, x_{k, n, r_{k}}(h)\right), \quad h \in \tilde{\mathcal{G}}[k]
$$

is an algebra isomorphism and $\operatorname{dim} \tilde{\mathcal{G}}[k]=r_{k}$.

Composing $\tilde{\Phi}_{k}: \tilde{\mathcal{G}}[k] \rightarrow \mathbb{F}^{r_{k}}$ with the canonical algebra isomorphism between $\tilde{\mathcal{G}}[k]$ and $\hat{\mathcal{G}}[k]$ given in (12), we have an algebra isomorphism $\hat{\Phi}_{k}: \hat{\mathcal{G}}[k] \rightarrow \mathbb{F}^{r_{k}}$. In particular, $\operatorname{dim} \hat{\mathcal{G}}[k]=$ $\operatorname{dim} \tilde{\mathcal{G}}[k]=r_{k}$.

Corollary 5.6. With the same assumption as in Lemma 5.5, the map

$$
\hat{\Phi}_{k, n}: \hat{\mathcal{G}}[k] \rightarrow \mathbb{F}^{r_{k}}, \quad \hat{\Phi}_{k, n}(h+\mathcal{K})=\left(\boldsymbol{x}_{k, n, 1}(h), \ldots, \boldsymbol{x}_{k, n, r_{k}}(h)\right), \quad h \in \hat{\mathcal{G}}[k]
$$

is an algebra isomorphism and $\operatorname{dim} \hat{\mathcal{G}}[k]=r_{k}$. 
Note that if $S \subseteq \mathbb{Z}_{>0}$ is finite and $|S|=k$, there are natural isomorphisms between $\tilde{\mathcal{G}}(S)$ and $\tilde{\mathcal{G}}[k]$ and also between $\hat{\mathcal{G}}(S)$ and $\hat{\mathcal{G}}[k]$, both resulting from any bijective map between $S$ and $[k]$. As a result, we conclude the following.

Corollary 5.7. Let $k \geq 0$ and $S \subseteq \mathbb{Z}_{>0}$ with $|S|=k$. Suppose there exists some $n=n_{k} \geq 2$ so that $r k_{S} T(f, k, n) \leq n-1$. Let $r_{k}=r k_{S} T(f, k, n)$. Then $\tilde{\mathcal{G}}(S) \cong \hat{\mathcal{G}}(S) \cong \mathbb{F}^{r}$ and $\operatorname{dim} \tilde{\mathcal{G}}(S)=\operatorname{dim} \hat{\mathcal{G}}(S)=$ $r_{k}$. In particular, the value $r_{k}$ is independent of the choice of $n$.

\subsection{One $\boldsymbol{n}$ implies for all $\boldsymbol{n}$}

Let $n_{k}$ retain the same meaning as in Lemma 5.5, and let $r=r_{k}=\operatorname{rk}_{\mathrm{S}}\left(T\left(f, k, n_{k}\right)\right)$. For any $h \in$ $\mathcal{G}[k]$, clearly $h=h U_{k}^{n_{k}-1}$ so $f(h)=f\left(h U_{k}^{n_{k}-1}\right)$. As $\mathbf{x}_{k, n_{k}, i}\left(U_{k}\right)=1,1 \leq i \leq r$,

$$
\begin{aligned}
f(h) & =f\left(h U_{k}^{n_{k}-1}\right)=\left(T\left(f, k, n_{k}\right)\right)\left(h, U_{k}, \ldots, U_{k}\right)=\sum_{i=1}^{r} a_{k, n_{k}, i}\left(\mathbf{x}_{k, n_{k}, i}\left(U_{k}\right)\right)^{n_{k}-1} \mathbf{x}_{k, n_{k}, i}(h) \\
& =\sum_{i=1}^{r} a_{k, n_{k}, i} \mathbf{x}_{k, n_{k}, i}(h)
\end{aligned}
$$

for any $h \in \mathcal{G}[k]$. Hence $f_{\mid \mathcal{G}[k]}=\sum_{i=1}^{r} a_{k, n_{k}, i} \mathbf{x}_{k, n_{k}, i}$, i.e. $f_{\mid \mathcal{G}[k]}$ is a linear combination of $r$ algebra homomorphisms $\mathbf{x}_{k, n_{k}, i}: \mathcal{G}[k] \rightarrow \mathbb{F}$, for $1 \leq i \leq r$. In particular, applying to the product $h_{1} \cdots h_{m}$, for any $m \geq 0$ and any $h_{1}, \ldots, h_{m} \in \mathcal{G}[k]$ (note that this $m$ is arbitrary, not constrained by $n_{k}$ ),

$$
f\left(h_{1} \cdots h_{m}\right)=\sum_{i=1}^{r} a_{k, n_{k}, i} \mathbf{x}_{k, n_{k}, i}\left(h_{1} \cdots h_{m}\right)=\sum_{i=1}^{r} a_{k, n_{k}, i} \mathbf{x}_{k, n_{k}, i}\left(h_{1}\right) \cdots \mathbf{x}_{k, n_{k}, i}\left(h_{m}\right) .
$$

(When $m=0$, we view it as $f\left(U_{k}\right)=\sum_{i=1}^{r} a_{k, n_{k}, i} \mathbf{x}_{k, n_{k}, i}\left(U_{k}\right)=\sum_{i=1}^{r} a_{k, n_{k}, i}$.) Hence

$$
T(f, k, m)=\sum_{i=1}^{r} a_{k, n_{k}, i} \mathbf{x}_{k, n_{k}, i}^{\otimes m}
$$

for all $m \geq 0$. (When $m=0, \quad(18)$ is still valid as $T(f, k, 0)=f\left(U_{k}\right)=\sum_{i=1}^{r} a_{k, n_{k}, i}=$ $\sum_{i=1}^{r} a_{k, n_{k}, i} \mathbf{x}_{k, n_{k}, i}^{\otimes 0}$ where the last equality is true as $\mathbf{x}_{k, n_{k}, i}^{\otimes 0}=1$.)

As shown before, $\mathbf{x}_{k, n_{k}, i}$ where $1 \leq i \leq r$ are linearly independent. Then by Lemma A.4 applied to (18), where the parameter $m$ in the tensor power is at least 2, we get $\operatorname{rk}_{S}(T(f, k, m))=r$ for all $m \geq 2$; and the decomposition (18) is actually unique up to a permutation for $m \geq 3$.

To summarize, this leads to the following.

Theorem 5.1. Let $k \geq 0$. Assume that for some $n=n_{k} \geq 2, r k_{S} T(f, k, n)=r \leq n-1$. Then the following hold:

(1). $r k_{\mathrm{S}} T(f, k, n)=r$ for every $n \geq 2$.

(2). There exist $r$ linearly independent algebra homomorphisms $\boldsymbol{x}_{i}: \mathcal{G}[k] \rightarrow \mathbb{F}$, and $a_{1}, \ldots, a_{r} \in$ $\mathbb{F} \backslash\{0\}$ such that $f_{\mid \mathcal{G}[k]}=\sum_{i=1}^{r} a_{i} x_{i} ;$ also for every $n \geq 0$,

$$
T(f, k, n)=\sum_{i=1}^{r} a_{i} x_{i}^{\otimes n}
$$

Moreover, for any $n \geq 3$, any expression of $T(f, k, n)$ as $\sum_{i=1}^{r} b_{i} y_{i}^{\otimes n}$, where $\boldsymbol{y}_{i}: \mathcal{G}[k] \rightarrow \mathbb{F}$ are linear maps, is a permutation of the sum in (19).

We remark that this is a non-trivial statement: The existence of some $n_{k}$ has produced a uniform expression for the tensors $T(f, k, n)$ all the way down to $n=0$. 


\subsection{Putting things together}

From now on, we assume that for every $k \geq 0$, there exist some $n=n_{k} \geq 2$ such that $\operatorname{rks}_{S} T(f, k, n) \leq n-1$. For every $k \geq 0$, we pick an arbitrary such $n$, call it $n_{k}$, and let $r_{k}=$ $\mathrm{rks}_{\mathrm{S}} T\left(f, k, n_{k}\right)$.

Having developed the theory in a more general setting, we can now follow the proof in [27] closely. As mentioned before, developing this theory in a more general setting is necessary because $f\left(K_{1}\right)=0$ is possible which makes the normalization step from [27] infeasible. Now the main difference from [27] starting from this point is that many of our derivations will additionally contain units of the form $u_{S}$ for various finite $S \subseteq \mathbb{Z}_{>0}$ because we cannot ensure that $u_{S}=u_{\emptyset}$. We will be interested in the idempotent elements of $\hat{\mathcal{G}}$. For two elements $p, q \in \hat{\mathcal{G}}$, we say that $q$ resolves $p$, if $p q=q$. We also say equivalently $p$ is resolved by $q$. It is clear that the binary relation resolves is antisymmetric and transitive and, when restricted to idempotents, reflexive. Furthermore, it is easy to see that the binary relation resolves on $\hat{\mathcal{G}}$ has the following properties:

1. The idempotent $0=\mathcal{K}$ resolves everything and $1=u_{\emptyset}=U_{\emptyset}+\mathcal{K}=K_{0}+\mathcal{K}$ is resolved by everything.

2. If $a b=0$ and $c$ resolves both $a$ and $b$, then $c=0$.

3. If $a$ resolves $b$, then $c$ resolves $a$ iff $c$ resolves $a b$.

In the algebra $\mathbb{F}^{r}(r \geq 0)$, the idempotents are 0-1 tuples in $\mathbb{F}^{r}$, and for idempotents $q=$ $\left(q_{1}, \ldots, q_{r}\right)$ and $p=\left(p_{1}, \ldots, p_{r}\right), q$ resolves $p$ iff $q_{i}=1$ implies $p_{i}=1$.

Let $S$ be a finite subset of $\mathbb{Z}_{>0}$ with $|S|=k$, and set $r=r_{k}$ as above. By Corollary 5.7, $\hat{\mathcal{G}}(S) \cong \mathbb{F}^{r}$ as algebras, so $\hat{\mathcal{G}}(S)$ has a (uniquely determined idempotent) basis $\mathcal{P}_{S}=\left\{p_{1}^{S}, \ldots, p_{r}^{S}\right\}$ such that $\left(p_{i}^{S}\right)^{2}=p_{i}^{S}$ and $p_{i}^{S} p_{j}^{S}=0$ for $i \neq j$. These correspond to the canonical basis $\left\{e_{i}\right\}_{1 \leq i \leq r}$ of $\mathbb{F}^{r}$ under this isomorphism. For $i \neq j$, we have $\left\langle p_{i}^{S}, p_{j}^{S}\right\rangle=f\left(p_{i}^{S} p_{j}^{S}\right)=0$. Furthermore, for all $1 \leq i \leq r$,

$$
f\left(p_{i}^{S}\right)=f\left(\left(p_{i}^{S}\right)^{2}\right)=\left\langle p_{i}^{S}, p_{i}^{S}\right\rangle \neq 0
$$

otherwise $\hat{\mathcal{G}}(S)$ contains a non-zero element orthogonal to $\hat{\mathcal{G}}(S)$ with respect to the bilinear form $\langle\cdot, \cdot\rangle$ restricted to $\hat{\mathcal{G}}(S) \times \hat{\mathcal{G}}(S)$, contradicting Lemma 5.1. We will call the elements $p_{i}^{S} \in \mathcal{P}_{S}$ basic idempotents.

We denote by $\mathcal{P}_{T, p}$ the set of all idempotents in $\mathcal{P}_{T}$ that resolve a given element $p \in \hat{\mathcal{G}}$. If $p \in \mathcal{P}_{S}$ and $S \subset T$ and $|T|=|S|+1$, then the number of elements in $\mathcal{P}_{T, p}$ will be called the degree of $p \in \mathcal{P}_{S}$, and denoted by $\operatorname{deg}(p)$. Obviously, this value is independent of which $(|S|+1)$-element superset $T$ of $S$ we take.

For any $q \in \hat{\mathcal{G}}(T)$, we have $q u_{T \backslash S}=q$. (Here by definition, $u_{T \backslash S}=U_{T \backslash S}+\mathcal{K} \in \hat{\mathcal{G}}(T \backslash S) \subseteq$ $\hat{\mathcal{G}}(T) \subseteq \hat{\mathcal{G}}$.) It follows that for any $S \subseteq T$ and $p \in \hat{\mathcal{G}}$, we have $q$ resolves $p$ iff $q$ resolves $p u_{T \backslash S}$, since $q p=q u_{T \backslash S} p=q p u_{T \backslash S}$. It is also important to point out that an element in $\hat{\mathcal{G}}(S)$ is an idempotent in $\hat{\mathcal{G}}(S)$ iff it is an idempotent in $\hat{\mathcal{G}}$.

Claim 9. Let $x$ be any idempotent element of $\hat{\mathcal{G}}(S)$. Then $x$ is the sum of exactly those idempotents in $\mathcal{P}_{S}$ that resolve it,

$$
x=\sum_{p \in \mathcal{P}_{S, x}} p .
$$

Proof. Let $k=|S|$, and $r=r_{k}$. By the isomorphism $\hat{\mathcal{G}}(S) \cong \mathbb{F}^{r}$, every 0-1 tuple $x=\left(x_{1}, \ldots, x_{r}\right) \in$ $\mathbb{F}^{r}$ is the $\operatorname{sum} \sum_{i=1}^{r} x_{i} e_{i}$. 
In particular

$$
u_{S}=\sum_{p \in \mathcal{P}_{S}} p
$$

since $u_{S} \in \hat{\mathcal{G}}(S)$ corresponds to the all-1 tuple in $\mathbb{F}^{r}$.

Claim 10. Let $S \subseteq T$ be two finite sets. Then every $q \in \mathcal{P}_{T}$ resolves exactly one element of $\mathcal{P}_{S}$.

Proof. Let $k^{\prime}=|T|$ and $r^{\prime}=r_{k^{\prime}}$. Consider the idempotents $p u_{T \backslash S}$ (which could be 0) under the isomorphism $\hat{\mathcal{G}}(T) \cong \mathbb{F}^{r^{\prime}}$, where $p \in \mathcal{P}_{S}$. We can write the 0-1 tuple corresponding to $p u_{T \backslash S}$ in $\mathbb{F}^{r^{\prime}}$ as the sum of those canonical basis 0 - 1 vectors. Recall that for any $q \in \mathcal{P}_{T}, q$ resolves $p$ iff $q$ resolves $p u_{T \backslash S}$. Note that $p u_{T \backslash S}$ must have disjoint positions with entry 1 for distinct $p \in \mathcal{P}_{S}$, and the sum $\sum_{p \in \mathcal{P}_{S}} p u_{T \backslash S}=u_{S} u_{T \backslash S}=u_{T}$ is the all-1 tuple in $\mathbb{F}^{r^{\prime}}$. Thus each $q \in \mathcal{P}_{T}$ resolves exactly one $p \in \mathcal{P}_{S}$.

Claim 11. Let $T$ and $U$ be finite sets, and let $S=T \cap U$. If $x \in \hat{\mathcal{G}}(T)$ and $y \in \hat{\mathcal{G}}(U)$, then

$$
f(x y)=f\left(\hat{\pi}_{S}(x) y\right) .
$$

Proof. For every $T$-labelled graph $G_{1}$ and $U$-labelled graph $G_{2}$, the graphs $G_{1} G_{2}$ and $\pi_{S}\left(G_{1}\right) G_{2}$ are isomorphic as unlabelled graphs. Hence $f\left(G_{1} G_{2}\right)=f\left(\pi_{S}\left(G_{1}\right) G_{2}\right)$ as $f$ ignores labels. Then we extend the equality from $\mathcal{P} \mathcal{L G}$ by linearity to $\mathcal{G}$ and after that proceed to the quotient $\hat{\mathcal{G}}=\mathcal{G} / \mathcal{K}$ using the definition of $f$ on $\hat{\mathcal{G}}$.

We remarked in $(20)$ that $f(p) \neq 0$ for any $p \in \mathcal{P}_{S}$.

Claim 12. Let $S \subseteq T$ be two finite sets. If $q \in \mathcal{P}_{T}$ resolves $p \in \mathcal{P}_{S}$, then

$$
\hat{\pi}_{S}(q)=\frac{f(q)}{f(p)} p .
$$

Proof. Note that $q \in \hat{\mathcal{G}}(T)$. Since $S \subseteq T$, it follows that $\hat{\pi}_{S}(q) \in \hat{\mathcal{G}}(S)$. Because the only element from $\hat{\mathcal{G}}(S)$ orthogonal to $\hat{\mathcal{G}}(S)$ with respect to the $\operatorname{dot} \operatorname{product}\langle\cdot, \cdot\rangle$ restricted to $\hat{\mathcal{G}}(S) \times \hat{\mathcal{G}}(S)$ is 0 (by Lemma 5.1), it suffices to show that both sides give the same dot product with every basis element in $\mathcal{P}_{S}$. For any $p^{\prime} \in \mathcal{P}_{S} \backslash\{p\}$, we have $p^{\prime} p=0$ so $p^{\prime} q=p^{\prime} p q=0$. By Claim 11, this implies that

$$
\left\langle p^{\prime}, \hat{\pi}_{S}(q)\right\rangle=f\left(p^{\prime} \hat{\pi}_{S}(q)\right)=f\left(p^{\prime} q\right)=0=\left\langle p^{\prime}, \frac{f(q)}{f(p)} p\right\rangle .
$$

Furthermore,

$$
\left\langle p, \hat{\pi}_{S}(q)\right\rangle=f\left(p \hat{\pi}_{S}(q)\right)=f(p q)=f(q)=\left\langle p, \frac{f(q)}{f(p)} p\right\rangle .
$$

This proves the claim.

Claim 13. Let $T$ and $U$ be finite sets and let $S=T \cap U$. Then for any $p \in \mathcal{P}_{S}, q \in \mathcal{P}_{T, p}$ and $r \in \hat{\mathcal{G}}(U)$ we have

$$
f(q r)=\frac{f(q)}{f(p)} f(r p)
$$

Proof. By Claims 11 and 12,

$$
f(q r)=f\left(\hat{\pi}_{S}(q) r\right)=\frac{f(q)}{f(p)} f(r p)
$$

Claim 14. Let $T$ and $U$ be finite sets and let $S=T \cap U$. If both $q \in \mathcal{P}_{T}, r \in \mathcal{P}_{U}$ resolve $p \in \mathcal{P}_{S}$, then $q r \neq 0$. 
Proof. By Claim 13,

$$
f(q r)=\frac{f(q)}{f(p)} f(r p)=\frac{f(q)}{f(p)} f(r) \neq 0 .
$$

Claim 15. If $S \subseteq T$, and $q \in \mathcal{P}_{T}$ resolves $p \in \mathcal{P}_{S}$, then $\operatorname{deg}(q) \geq \operatorname{deg}(p)$.

Proof. It suffices to show this in the case when $|T|=|S|+1$. Let $U \subset \mathbb{Z}_{>0}$ be any $(|S|+1)$ element superset of $S$ different from $T$; suppose this is the disjoint union $U=S \sqcup\{a\}, a \notin T$. Let $Y$ be the set of elements in $\mathcal{P}_{U}$ resolving $p u_{\{a\}}$ (equivalently, resolving $p$, because every $r \in \mathcal{P}_{U}$ resolves $u_{\{a\}}$ as $\left.a \in U\right)$. Then $p u_{\{a\}}=\sum_{r \in Y} r$ by Claim 9. Here $|Y|=\operatorname{deg}(p)$. Furthermore, we have

$$
\sum_{r \in Y} q r=q \sum_{r \in Y} r=q p u_{\{a\}}=q u_{\{a\}} .
$$

Each term $q r$ on the left hand side is non-zero by Claim 14, and since the terms are all idempotent, each of them is a sum of one or more elements of $\mathcal{P}_{T \cup U}$. Furthermore, if $r, r^{\prime} \in Y\left(r \neq r^{\prime}\right)$, then we have the orthogonality relation

$$
(q r)\left(q r^{\prime}\right)=q\left(r r^{\prime}\right)=0,
$$

so the sets of basic idempotents of $\mathcal{P}_{T \cup U}$ in the expansion of each term are pairwise disjoint. Therefore the expansion $\sum_{r \in Y} q r$ in $\mathcal{P}_{T \cup U}$ contains at least $|Y|=\operatorname{deg}(p)$ terms. On the right hand side of (22), for any $z \in \mathcal{P}_{T \cup U}, z$ resolves $q$ iff $z$ resolves $q u_{\{a\}}$ since $a \in U$. Thus the number of terms in the expansion of $q \mathcal{u}_{\{a\}}$ in the basis $\mathcal{P}_{T \cup U}$ is precisely deg $(q)$ by definition. Thus, $\operatorname{deg}(q) \geq$ $|Y|=\operatorname{deg}(p)$. The claim is proved.

\subsection{Bounding the expansion}

At this point, we finally assume that all the conditions of Theorem 3.3 are satisfied, i.e. $f\left(K_{0}\right)=1$ and there is an integer $q \geq 0$ such that for every $k \geq 0$ there exists $n=n_{k} \geq 2$ satisfying $r_{k, n}=$ $\operatorname{rk}_{\mathrm{S}} T(f, k, n) \leq \min \left(n-1, q^{k}\right)$. In particular $r_{0, n} \leq q^{0}=1$ for $n=n_{0} \geq 2$. Clearly, $r_{0, n} \neq 0$ since $f\left(K_{0}\right)=1$ so $r_{0, n}=1$. By Proposition 3.1, $f$ is multiplicative.

Next, from $f\left(K_{0}\right)=1$, we have $U_{\emptyset}=K_{0} \notin \mathcal{K}$ so that $u_{\emptyset}=U_{\emptyset}+\mathcal{K}$ is a non-zero identity in $\hat{\mathcal{G}}(\emptyset)=\hat{\mathcal{G}}[0] \neq 0$. As $u_{\emptyset}$ is the sum of all basic idempotents in $\mathcal{G}(\emptyset)$ we infer that $\mathcal{P}_{\emptyset} \neq 0$. Hence there is at least one basic idempotent.

If for any finite $S \subseteq \mathbb{Z}_{>0}$, a basic idempotent $p \in \mathcal{P}_{S}$ has degree $D \geq 0$, then for any superset $T \subseteq \mathbb{Z}_{>0}$ of $S$ with $|T|=|S|+1$, there are $D$ basic idempotents resolving $p$. Let $S \subseteq T, t=|T \backslash S|$ and $T \backslash S=\left\{u_{1}, u_{2}, \ldots, u_{t}\right\}$. For each $1 \leq i \leq t$, we can pick $D$ basic idempotents $q_{j}^{u_{i}} \in \mathcal{P}_{S \cup\left\{u_{i}\right\}}$ resolving $p$, where $1 \leq j \leq D$. For any mapping $\phi:\{1, \ldots, t\} \rightarrow\{1, \ldots, D\}$, we can form the product $q_{\phi}=\prod_{i=1}^{t} q_{\phi(i)}^{u_{i}}$. If $t=0$, we assume $q_{\phi}=p$. These are clearly idempotents resolving $p$. If $\phi \neq \phi^{\prime}$ then for some $i$, we have the orthogonality relation $q_{\phi(i)}^{u_{i}} q_{\phi^{\prime}(i)}^{u_{i}}=0$. Thus $q_{\phi} q_{\phi^{\prime}}=0$. Also by applying Claim $13 t$ times,

$$
f\left(q_{\phi}\right)=f\left(q_{\phi} p\right)=f\left(\prod_{i=1}^{t} q_{\phi(i)}^{u_{i}} p\right)=\left(\prod_{i=1}^{t} \frac{f\left(q_{\phi(v)}^{u_{i}}\right)}{f(p)}\right) f(p) \neq 0,
$$

and so $q_{\phi} \neq 0$. Thus the set $\left\{q_{\phi} \mid \phi:\{1, \ldots, t\} \rightarrow\{1, \ldots, D\}\right\}$ is linearly independent. This implies that the dimension of $\hat{\mathcal{G}}(T)$ over $\mathbb{F}$ is at least $D^{t}=D^{|T|-|S|}$. But by Corollary 5.7 and the hypothesis of Theorem 3.3 we also have the upper bound $q^{|T|}$. If $D>q$, this leads to a contradiction if $|T|$ is large. It follows that $D \leq q$, i.e. the degrees of basic idempotents for any $S$ and any $p \in \mathcal{P}_{S}$ are bounded by $q$. Let $D \geq 0$ denote the maximum degree over all such $S$ and $p \in \mathcal{P}_{S}$, and suppose it is attained at some particular $S$ and $p \in \mathcal{P}_{S}$. We now fix this $S$ and $p$. Note that for the existence of $D$ we also use the existence of a basic idempotent. 
For $u \in \mathbb{Z}_{>0} \backslash S$, let $q_{1}^{u}, \ldots, q_{D}^{u}$ denote the elements of $\mathcal{P}_{S \cup\{u\}}$ resolving $p$. Note that for $u, v \in$ $\mathbb{Z}_{>0} \backslash S$, there is a natural isomorphism between $\hat{\mathcal{G}}(S \cup\{u\})$ and $\hat{\mathcal{G}}(S \cup\{v\}$ ) (induced by the map that fixes $S$ pointwise and maps u to $v$ ), and we may choose the indexing so that $q_{i}^{u}$ corresponds to $q_{i}^{v}$ under this isomorphism.

Now for any finite set $T \supseteq S$ all basic idempotents in $\mathcal{P}_{T}$ that resolve $p$ can be described. To describe these, let $V=T \backslash S$, and for every map $\phi: V \rightarrow\{1, \ldots, D\}$, we define as before

$$
q_{\phi}=\prod_{v \in V} q_{\phi(v)}^{v}
$$

We have shown that these are linearly independent.

\section{Claim 16.}

$$
\mathcal{P}_{T, p}=\left\{q_{\phi}: \phi \in\{1, \ldots, D\}^{V}\right\}
$$

Proof. We prove this by induction on the cardinality of $V=T \backslash S$. For $|V|=0,1$ the assertion is trivial. Suppose that $|V|>1$. Pick any $u \in V$, let $U=S \cup\{u\}$ and $W=T \backslash\{u\}$; thus $U \cap W=S$. By the induction hypothesis, the basic idempotents in $\mathcal{P}_{W}$ resolving $p$ are elements of the form $q_{\psi}$, for $\psi \in\{1, \ldots, D\}^{W}$.

Let $r$ be one of these. By Claim $14, r q_{i}^{u} \neq 0$ for any $1 \leq i \leq D$, and clearly resolves $r$. We can write $r q_{i}^{u}$ as a sum of basic idempotents in $\mathcal{P}_{T}$ resolving it, and it is easy to see that these also resolve $r$ (as resolve is transitive). For each $r q_{i}^{u}$ the sum is non-empty as $r q_{i}^{u} \neq 0$. Furthermore, the sets of basic idempotents occurring in the expressions for $r q_{i}^{u}$ and $r q_{j}^{u}(i \neq j)$ are disjoint; this follows from item 5.4 stated at the beginning of this subsection, and $q_{i}^{u} q_{j}^{u}=0$. If the sum for any $r q_{i}^{u}$ has more than one basic idempotent, then $r$ would have degree $>D$, violating the maximality of $D$. So each $r q_{i}^{u}$ must be a basic idempotent in $\mathcal{P}_{T}$ itself.

Each $r \in \mathcal{P}_{W}$ resolves $p$ iff $r$ resolves $p u_{W}$. Hence $p u_{W}=\sum_{r \in \mathcal{P}_{W, p}} r$. Also $p u_{\{u\}}=\sum_{i=1}^{D} q_{i}^{u}$. Therefore we have

$$
p u_{T}=p u_{W} p u_{\{u\}}=\sum_{r \in \mathcal{P}_{W, p}, 1 \leq i \leq D} r q_{i}^{u},
$$

i.e. the basic idempotents $r q_{i}^{u}\left(r \in \mathcal{P}_{W, p}, 1 \leq i \leq D\right)$ form the set of basic idempotents in $\mathcal{P}_{T}$ resolving $p u_{T}$, which is equivalent to resolving $p$. It follows that these are all the elements of $\mathcal{P}_{T, p}$. This proves the claim.

It is immediate from the definition that an idempotent $q_{\phi}$ resolves $q_{i}^{v} u_{V}$ (equivalently $q_{i}^{v}$ ) iff $\phi(v)=i$. Hence it also follows that

$$
q_{i}^{v} u_{V}=\sum_{\phi: \phi(v)=i} q_{\phi}
$$

By the same reason, it also follows that for $u, v \in V, u \neq v$, and any $1 \leq i, j \leq D$,

$$
q_{i}^{u} q_{j}^{v} u_{V}=\sum_{\substack{\phi: \phi(u)=i \\ \phi(v)=j}} q_{\phi}
$$

\subsection{Constructing the target graph}

Now we can define $H$ as follows. Let $S$ and $p$ be fixed as above. For any $u \in \mathbb{Z}_{>0} \backslash S$, let $\left\{q_{1}^{u}, \ldots, q_{D}^{u}\right\}$ be defined as in subsection 5.5 .

Let $H$ be the looped complete graph on $V(H)=\{1, \ldots, D\}$. We have to define the node weights and edge weights. For every $i \in V(H)$, let

$$
\alpha_{i}=\frac{f\left(q_{i}^{u}\right)}{f(p)}
$$


be the weight of node $i .^{5}$ This definition does not depend on the choice of $u$, because if $v \in \mathbb{Z}_{>0} \backslash S$ and $v \neq u$, then the isomorphism from $\hat{\mathcal{G}}(S \cup\{u\})$ to $\hat{\mathcal{G}}(S \cup\{v\})$ (induced by the map that fixes $S$ and maps u to $v$ ), will send $q_{i}^{u}$ to $q_{i}^{v}$.

Let $u, v \in \mathbb{Z}_{>0} \backslash S, u \neq v$, and let $W=S \cup\{u, v\}$. Let $K_{u v}$ denote the graph on the vertices $u$ and $v$ that are correspondingly labelled $u$ and $v$, and has only one edge connecting $u$ and $v$. Let $k_{u v}=K_{u v}+\mathcal{K}$ denote the corresponding element of $\hat{\mathcal{G}}(\{u, v\})$. We can express $p k_{u v}$ as a linear combination of the basic idempotents from $\mathcal{P}_{W}$. Note that $r \in \mathcal{P}_{W}$ resolves $p$ iff $r$ resolves $p u_{\{u, v\}}$, thus $p u_{\{u, v\}}=\sum_{r \in \mathcal{P}_{W, p}} r$. So if $r^{\prime} \in \mathcal{P}_{W} \backslash \mathcal{P}_{W, p}$, we have $r^{\prime} p=r^{\prime} u_{\{u, v\}} p=0$. Thus $r^{\prime} p k_{u v}=0$. It follows that $p k_{u v}$ is a linear combination of the basic idempotents from the subset $\mathcal{P}_{W, p}$. We write this unique expression

$$
p k_{u v}=\sum_{i, j=1}^{D} \beta_{i j} q_{i}^{u} q_{j}^{v}
$$

This defines (by the uniqueness) the weight $\beta_{i j}$ of the edge $i j$. Note that $\beta_{i j}=\beta_{j i}$ for all $i, j$, since $p k_{u v}=p k_{v u}$.

We prove that this weighted graph $H$ gives the desired homomorphism function.

Claim 17. For every finite graph $\mathrm{G}, f(G)=\operatorname{hom}(G, H)$.

Proof. Let $V$ be a finite subset of $\mathbb{Z}_{>0}$ disjoint from $S$ of cardinality $|V(G)|$. We label $V(G)$ by $V$ thus making $G$ a $V$-labelled graph, so now $G \in \mathcal{G}(V)$. Since $f$ ignores labels, we may identify $V$ and $V(G)$, and assume $V(G)=V$. Now we take $T=S \sqcup V$, thus $V=T \backslash S$. This defines $q_{\phi}$ as in subsection 5.5. By (25), we have for each pair $u, v$ of distinct elements of $V(G)$,

$$
p k_{u v} u_{V}=\sum_{i, j \in V(H)} \beta_{i j} q_{i}^{u} q_{j}^{v} u_{V}=\sum_{i, j \in V(H)} \beta_{i, j} \sum_{\begin{array}{r}
\phi: \phi(u)=i \\
\phi(v)=j
\end{array}} q_{\phi}=\sum_{\phi \in V(H)^{V}} \beta_{\phi(u), \phi(v)} q_{\phi} .
$$

Here to define the set of mappings $\phi$ we take $T=S \sqcup V$, thus $V=T \backslash S$. Then the last equality follows from the fact that

$$
\{\phi: V \rightarrow V(H)\}=\bigsqcup_{i, j \in V(H)}\left\{\phi \in V(H)^{V}: \phi(u)=i, \phi(v)=j\right\}
$$

is a partition. Let $g=G+\mathcal{K}$ be the corresponding element of $G$ in $\hat{\mathcal{G}}(V)$. Clearly $G=$ $\left(\prod_{u v \in E(G)} K_{u, v}\right) U_{V}$ so $g=\left(\prod_{u v \in E(G)} k_{u v}\right) u_{V}$. (When $E(G)=\emptyset$, we view it as $G=U_{V}$ so $g=u_{V}$.) We have $f(G)=f(g)$ by the definition of $f$ on $\hat{\mathcal{G}}$. Also note that $g u_{V}=g$ so $g=g u_{V}^{|E(G)|}$. Also, $p$ is an idempotent so $p=p^{|E(G)|+1}$. Then

$$
p g=p^{|E(G)|+1} g u_{V}^{|E(G)|}=p\left(\prod_{u v \in E(G)} p k_{u v} u_{V}\right) u_{V}=p\left(\prod_{u v \in E(G)}\left(\sum_{\phi \in V(H)^{V}} \beta_{\phi(u), \phi(v)} q_{\phi}\right)\right) u_{V} .
$$

(When $E(G)=\emptyset$, this is simply $p g=p u_{V}$.) Note that when we expand the product of sum as a sum of products, for any two edges $u v \in E(G)$ and $u^{\prime} v^{\prime} \in E(G)$, if the mappings $\phi$ and $\phi^{\prime} \in V(H)^{V}$ (in the respective sums) disagree on any vertex of $V=V(G)$, the product $q_{\phi} q_{\phi^{\prime}}=0$. This implies that in the sum-of-products expression we only sum over all $\phi \in V(H)^{V}$ (and not over the $|E(G)|$ tuples of these). Also $q_{\phi}$ resolves $p$, so $p q_{\phi}=q_{\phi}$. Moreover, each $q_{\phi} \in \hat{\mathcal{G}}(S \cup V)$ so $q_{\phi} u_{V}=q_{\phi}$. This

\footnotetext{
${ }^{5}$ For $\mathbb{F}=\mathbb{R}$, if we require the positive semidefiniteness of the connection matrices $M(f, k)$ for $k \geq 0$, then since $p$ and $q_{i}^{u}$ are basic idempotents, $f(p)=f\left(p^{2}\right)>0$ and similarly $f\left(q_{i}^{u}\right)>0$. Thus $\alpha_{i}>0$, and so we recover the positive vertex weight case; see [27].
} 
implies that

$$
p g=\left(\sum_{\phi: V \rightarrow V(H)}\left(\prod_{u v \in E(G)} \beta_{\phi(u), \phi(v)}\right) q_{\phi}\right) u_{V}=\sum_{\phi: V \rightarrow V(H)}\left(\prod_{u v \in E(G)} \beta_{\phi(u), \phi(v)}\right) q_{\phi} .
$$

(When $E(G)=\emptyset$, we view it as $p g=p u_{V}=\sum_{\phi: V \rightarrow V(H)} q_{\phi}=\sum_{\phi: V \rightarrow V(H)} \quad\left(\prod_{u v \in E(G)}\right.$ $\left.\beta_{\phi(u), \phi(v)}\right) q_{\phi}$ which is true by Claims 9 and 16 with $\dot{T}=S \cup V$ and the fact that an element $h \in \hat{\mathcal{G}}(S \cup V)$ resolves $p$ iff $h$ resolves $p u_{V}$.) Note that $p \in \hat{\mathcal{G}}(S)$ and has a representative in $\mathcal{G}(S)$ as a linear combination of labelled graphs from $\mathcal{P} \mathcal{L} \mathcal{G}(S), g \in \hat{\mathcal{G}}(V)$ has the representative $G \in \mathcal{P} \mathcal{L} \mathcal{G}(V) \subseteq \mathcal{G}(V)$, and $S \cap V=\emptyset$. Hence $f(p) f(g)=f(p g)$, as $f$ is multiplicative, $f$ ignores labels and also by the definition of $f$ on $\hat{\mathcal{G}}$. Therefore by (23) and (24),

$$
\begin{aligned}
f(p) f(G) & =f(p) f(g)=f(p g)=\sum_{\phi: V \rightarrow V(H)}\left(\prod_{u v \in E(G)} \beta_{\phi(u), \phi(v)}\right) f\left(q_{\phi}\right) \\
& =\sum_{\phi: V \rightarrow V(H)}\left(\prod_{u v \in E(G)} \beta_{\phi(u), \phi(v)}\right)\left(\prod_{v \in V(G)} \alpha_{\phi(v)}\right) f(p),
\end{aligned}
$$

Since $f(p) \neq 0$, we can cancel it on both sides, and complete the proof.

Remark 5.1. Note that if $D=0$, then from the proof we get that $H$ is the empty graph, so $f(G)=0$ unless $G=K_{0}$ (the empty graph) and $f\left(K_{0}\right)=1$. After that, we trivially get $\hat{\mathcal{G}}(T)=0$ for any $T \neq \emptyset$ and $\hat{\mathcal{G}}(\emptyset) \cong \mathbb{F}$ as algebras. However, $p \in \mathcal{P}_{S}$ so it follows that $S=\emptyset$. Therefore by the previous isomorphism $\mathcal{P}_{\emptyset}=\{p\}$ so $p$ is the only basic idempotent in $\hat{\mathcal{G}}(\emptyset)$ and so in the entire $\hat{\mathcal{G}}$.

\section{Extensions}

So far we have allowed $G$ to have multiple edges but no loops as is the standard definition. We can extend the results in this paper to more general graphs. If we allow (multiple) loops in $G$, we can show that the (multiplicative) graph parameter $f(G)=a^{\# l o o p s(G)}$ where $1 \neq a \in \mathbb{F}$ ( $a$ can be 0 ) cannot be expressed as a GH function, even though its connection tensors $T(f, k, n)$ all have symmetric rank 1 and $f\left(K_{0}\right)=1$. To get the corresponding representation theorem for graphs with (multiple) loops, in the target graph $\mathrm{H}$ each loop $e$ attached to a vertex $i$ must have two weights: $\beta_{i i}$ which is used when a nonloop edge of $G$ is mapped onto $e$, and the other, say $\gamma_{i}$, when a loop of $G$ is mapped onto $e$. In this extended model we have the following:

- The main expressibility results Theorems 3.1, 3.2 and 3.3 remain true with the proof from Section 5 carrying over to this model with slight adjustments.

- The GH inexpressibility results from Section 4 remain true as the provided proofs involve only simple loopless graphs. (For \#VERTEX-DisJOINT-CYCLE-COVER (vdcc), a loop at a vertex is considered a cycle cover of that vertex; this is consistent with the definition in Holant problems.)

- The results from Subsection 4.4 on bounded degree graphs remain true in the sense that the inexpressibility results hold if we allow $\gamma_{i}$ to be arbitrary (again, since only simple loopless graphs were used in the proof), while the expressibility holds even with the stronger requirement $\gamma_{i}=\beta_{i i}$.

Analogously, a GH expressibility criterion can be stated and proved within the framework of directed GH with minor adjustments, too. We note that generalizations of results in [27] were given in [40] to a more general model which captures directed graphs, hypergraphs, etc. We expect 
that it is possible to generalize the GH expressibility criterion in this paper for arbitrary fields to this more general model in a similar way as done in [40].

\section{A. Appendix}

\section{A.1 Multilinear algebra}

We prove some statements we need about tensors. We assume that the reader is familiar with the definition of a multilinear function, tensor product and dual space. It is good to start with coordinate-free definitions because it allows a succinct notation. But we will mostly use coordinates. The results are concrete, and they can be understood without too much formalism.

Unless stated otherwise, we do not impose a particular order on the rows and columns of matrices, or coordinates of tensors. The vector spaces may be infinite dimensional; and this infinite dimensionality is a main technical point that causes some complications.

The tensor product of vector spaces $V_{1}, \ldots, V_{n}$ over $\mathbb{F}$ is denoted by $V_{1} \otimes \cdots \otimes V_{n}$ or $\bigotimes_{i=1}^{n} V_{i}$. Elements of $\bigotimes_{i=1}^{n} V_{i}$ are called order- $n$ tensors. When $V_{i}=V$ for $1 \leq i \leq n$, we denote the tensor product by $V^{\otimes n}$. (By convention $V^{\otimes 0}=\mathbb{F}$, and $v^{\otimes 0}=1 \in \mathbb{F}$.) Define a group action by $S_{n}$ on $V^{\otimes n}$ induced by $\sigma\left(\otimes_{i=1}^{n} v_{i}\right)=\bigotimes_{i=1}^{n} v_{\sigma(i)}$. Recall that $V^{\otimes n}$ consists of finite linear combinations of such terms. We call a tensor $A \in V^{\otimes n}$ symmetric if $\sigma(A)=A$ for all $\sigma \in S_{n}$, and denote by $\operatorname{Sym}^{n}(V)$ the set of symmetric tensors in $V^{\otimes n}$. As $\mathbb{F}$ may have finite characteristic $p$, the usual symmetrizing operator from $V^{\otimes n}$ to $\operatorname{Sym}^{n}(V)$, which requires division by $n$ !, is in general not defined.

Multilinear functions on $\prod_{i=1}^{n} V_{i}$ can be naturally identified with the dual space $\left(\bigotimes_{i=1}^{n} V_{i}\right)^{*}$ of linear functions on $\bigotimes_{i=1}^{n} V_{i}$, induced by $f \mapsto f^{\prime}$, satisfying $f^{\prime}\left(\otimes_{i=1}^{n} v_{i}\right)=f\left(v_{1}, \ldots, v_{n}\right)$. Moreover, $\bigotimes_{i=1}^{n} V_{i}^{*}$ canonically embeds into $\left(\bigotimes_{i=1}^{n} V_{i}\right)^{*}$ via $\left(\otimes_{i=1}^{n} f_{i}\right)\left(\otimes_{i=1}^{n} v_{i}\right)=\prod_{i=1}^{n} f_{i}\left(v_{i}\right)$. A special case is that $\left(V^{*}\right)^{\otimes n}$ embeds into $\left(V^{\otimes n}\right)^{*}$. If all $V_{i}$ 's are finite dimensional then this embedding is an isomorphism. However, if $V_{i}$ are infinite dimensional, this embedding is not surjective. To see this, consider $V^{\otimes 2}$ where $V$ is the linear span of $\left\{e_{i} \mid i \in \mathbb{Z}_{>0}\right\}$, where $e_{i}$ is the 0 - 1 vector indexed by $\mathbb{Z}_{>0}$ with a single 1 at the $i$ th position. Let $f \in\left(V^{\otimes 2}\right)^{*}$ be such that $f\left(e_{i} \otimes e_{j}\right)=\delta_{i j}$, which is 1 if $i=j$ and 0 otherwise. Then there is no tensor $T \in\left(V^{*}\right)^{\otimes 2}$ that embeds as $f$. Indeed, any $T \in\left(V^{*}\right)^{\otimes 2}$ is, by definition, a finite sum $T=\sum_{1<k<n} c_{k} f_{k} \otimes g_{k}$. If $T$ were to embed as $f$, then consider the $n \times n$ matrix where the $(i, j)$ entry is $f\left(e_{i} \otimes e_{j}\right)$, which is the identity matrix $I_{n}$ of rank $n$. However $T\left(e_{i} \otimes e_{j}\right)=\sum_{1 \leq k<n} c_{k} f_{k}\left(e_{i}\right) \cdot g_{k}\left(e_{j}\right)$, and so the matrix for the embedded $T$ has rank $<n$, being a sum of $n-1$ matrices of rank $\leq 1$.

Let $A_{i}: V_{i} \rightarrow U_{i}(1 \leq i \leq n)$ be linear maps of vector spaces. They induce a homomorphism $\left(\bigotimes_{i=1}^{n} U_{i}\right)^{*} \rightarrow\left(\bigotimes_{i=1}^{n} V_{i}\right)^{*}$ via $f \mapsto g$, satisfying $g\left(\otimes_{i=1}^{n} v_{i}\right)=f\left(\otimes_{i=1}^{n} A_{i} v_{i}\right)$.

If $V_{i}$ are vector subspaces of $U_{i}$, then $\bigotimes_{i=1}^{n} V_{i}$ canonically embeds in $\bigotimes_{i=1}^{n} U_{i}$. In particular, if $V \subseteq U$, then $V^{\otimes n}$ and $\operatorname{Sym}^{n}(V)$ canonically embed in $U^{\otimes n}$ and $\operatorname{Sym}^{n}(U)$ respectively. Under this embedding $\operatorname{Sym}^{n}(V)=\operatorname{Sym}^{n}(U) \cap V^{\otimes n}$. We will also denote the space of symmetric $n$-fold multilinear functions on $V$ by $\operatorname{Sym}\left(\left(V^{\otimes n}\right)^{*}\right)$, i.e. the functions from $\left(V^{\otimes n}\right)^{*}$ that are symmetric. We have $\left(V^{*}\right)^{\otimes n} \cap \operatorname{Sym}\left(\left(V^{\otimes n}\right)^{*}\right)=\operatorname{Sym}^{n}\left(V^{*}\right)$.

In this paper, we are interested in vector spaces of the form $V=\bigoplus_{i \in \mathcal{I}} \mathbb{F}_{i}$, or just $\bigoplus_{\mathcal{I}} \mathbb{F}$, where $\mathcal{I}$ is an (index) set and each $\mathbb{F}_{i}, i \in \mathcal{I}$, is a copy of $\mathbb{F}$ indexed by $i$. In this case $V$ has a basis $\left\{e_{i} \mid i \in \mathcal{I}\right\}$, and a vector $v \in V$ has finitely many nonzeros in this basis. Note that for infinite $\mathcal{I}$ this is a proper subset of $\mathbb{F}^{\mathcal{I}}$, and in particular $\left\{e_{i} \mid i \in \mathcal{I}\right\}$ is not a basis ${ }^{6}$ for $\mathbb{F}^{\mathcal{I}}$. For $V=\bigoplus_{\mathcal{I}} \mathbb{F}$, the dual space $V^{*}$ can be identified with $\mathbb{F}^{\mathcal{I}}$ via $f \mapsto\left(f\left(e_{i}\right)\right)_{i \in \mathcal{I}}$. For $V=\bigoplus_{\mathcal{I}} \mathbb{F}$, we have $V^{\otimes n}=\bigoplus_{\mathcal{I}^{n}} \mathbb{F}$, and $\left(V^{\otimes n}\right)^{*}$ can be identified with $\mathbb{F}^{\mathcal{I}^{n}}$, the $n$-dimensional arrays. We can view $\operatorname{Sym}\left(\mathbb{F}^{\mathcal{I}^{n}}\right)=\operatorname{Sym}\left(\left(V^{\otimes n}\right)^{*}\right)$ as symmetric arrays, i.e. arrays in $\mathbb{F}^{\mathcal{I}^{n}}$ that are invariant under permutations from $S_{n}$, with respect to the basis $\left\{e_{i} \mid i \in \mathcal{I}\right\}$ of $V=\bigoplus_{i \in \mathcal{I}} \mathbb{F}_{i}$.

\footnotetext{
${ }^{6}$ Of course every vector space has a basis; however, this requires Zorn's Lemma so the proof is nonconstructive. In this paper, our results are constructive usually working with an explicitly given basis.
} 
Any $A \in \bigotimes_{j=1}^{n} V_{j}$, where $n \geq 1$, can be expressed as a finite sum

$$
A=\sum_{i=1}^{r} \mathbf{v}_{i 1} \otimes \cdots \otimes \mathbf{v}_{i n}, \quad \mathbf{v}_{i j} \in V_{j} .
$$

The least $r \geq 0$ for which $A$ has such an expression is called the $\operatorname{rank}$ of $A$, denoted by $\operatorname{rank}(A)$. Clearly, $A=0$ iff $\operatorname{rank}(A)=0$. If $r=\operatorname{rank}(A)>0$ then in any such expression of $A$ of $r$ terms all vectors $\mathbf{v}_{i j} \neq 0$. When $n=0, \bigotimes_{j=1}^{n} V_{j}$ is $\mathbb{F}$, and we define $\operatorname{rank}(A)=1$ for $A \neq 0$ and $\operatorname{rank}(A)=0$ for $A=0$.

Similarly, for $A \in \operatorname{Sym}^{n}(V)$ we define the symmetric rank of $A$ to be the least $r \geq 0$ for which $A$ can be expressed as

$$
A=\sum_{i=1}^{r} \lambda_{i} \mathbf{v}_{i}^{\otimes n}, \quad \lambda_{i} \in \mathbb{F}, \mathbf{v}_{i} \in V,
$$

and is denoted by $\operatorname{rk}_{\mathrm{S}}(A)$. If there is no such decomposition we define $\operatorname{rk}_{\mathrm{S}}(A)=\infty$. If $\operatorname{rk}_{\mathrm{S}}(A)<\infty$ then in any such expression of $A$ as a sum of $\operatorname{rks}(A)$ terms all $\lambda_{i} \neq 0$, all $\mathbf{v}_{i} \neq 0$ and are pairwise linearly independent. We show in Lemma A.6 that for infinite $\mathbb{F}, \operatorname{rk}_{\mathrm{S}}(A)<\infty$ for all $A \in \operatorname{Sym}^{n}(V)$.

We also need to refer to the rank of functions in $\left(\bigotimes_{i=1}^{n} V_{i}\right)^{*}$. As mentioned before $\bigotimes_{i=1}^{n} V_{i}^{*}$ is embedded as a subspace of $\left(\bigotimes_{i=1}^{n} V_{i}\right)^{*}$. For a function $F \in\left(\bigotimes_{i=1}^{n} V_{i}\right)^{*}$, where $n \geq 1$, we define the rank of the function $F$ to be $\infty$ if $F \notin \bigotimes_{i=1}^{n} V_{i}^{*}$, and if $F \in \bigotimes_{i=1}^{n} V_{i}^{*}$, the rank of $F$ is the least $r$ for which $F$ can be written as

$$
F=\sum_{i=1}^{r} \mathbf{f}_{i 1} \otimes \cdots \otimes \mathbf{f}_{i n}, \quad \mathbf{f}_{i j} \in V_{j}^{*} .
$$

When $n=0,\left(\bigotimes_{j=1}^{n} V_{j}\right)^{*}$ is $\mathbb{F}^{*} \cong \mathbb{F}$, and we define $\operatorname{rank}(F)=1$ for $F \neq 0$ and $\operatorname{rank}(F)=0$ for $F=$ 0 . The symmetric $\operatorname{rank} \operatorname{rk}_{S}(F)$ of $F \in \operatorname{Sym}\left(\left(V^{\otimes n}\right)^{*}\right)$ is similarly defined. It is $\infty$ if $F \notin \operatorname{Sym}^{n}\left(V^{*}\right)$. For $F \in \operatorname{Sym}^{n}\left(V^{*}\right)$, we define $\mathrm{rks}_{\mathrm{S}}(F)$ to be the least $r$ such that

$$
F=\sum_{i=1}^{r} \lambda_{i} \mathbf{f}_{i}^{\otimes n}, \quad \lambda_{i} \in \mathbb{F}, \quad \mathbf{f}_{i} \in V^{*},
$$

if such an expression exists; $\mathrm{rk}_{\mathrm{S}}(F)=\infty$ otherwise. By the same Lemma A.6 for infinite $\mathbb{F}$, we have $\operatorname{rk}_{\mathrm{S}}(F)<\infty$ for all $F \in \operatorname{Sym}^{n}\left(V^{*}\right)$.

Basically, the rank of a multilinear function is just an extension of the tensor rank from $\bigotimes_{i=1}^{n} V_{i}^{*}$ to $\left(\bigotimes_{i=1}^{n} V_{i}\right)^{*}$. Similarly the symmetric rank is the extension from $\operatorname{Sym}^{n}\left(V^{*}\right)$ to $\operatorname{Sym}\left(\left(V^{\otimes n}\right)^{*}\right)$. Clearly for all symmetric $A, \operatorname{rank}(A) \leq \operatorname{rk}_{\mathrm{S}}(A)$. Both $\operatorname{rank}$ and $\mathrm{rk}_{\mathrm{S}}$ are unchanged when moving from $\otimes V_{i}$ to $\otimes U_{i}$, if $V_{i} \subseteq U_{i}$.

Lemma A.1. The vectors $\boldsymbol{x}_{1}, \ldots, \boldsymbol{x}_{r} \in \mathbb{F}^{\mathcal{I}}$ are linearly independent iff in the $r \times \mathcal{I}$ matrix formed by $\boldsymbol{x}_{1}, \ldots, \boldsymbol{x}_{r}$ as rows there exists a non-zero $r \times r$ minor.

Proof. $\Leftarrow$ is obvious, so let us prove $\Rightarrow$. Let $R \subseteq[r]$ be a maximal subset satisfying the property that for some finite subset $C \subseteq \mathcal{I}$ the set of vectors $\left\{\left.\mathbf{x}_{i}\right|_{C}: i \in R\right\}$ is linearly independent, where $\left.\mathbf{x}_{i}\right|_{C}$ is the restriction of $\mathbf{x}_{i}$ to $C$. Suppose linear independence is achieved by $C$ for $R$. Then it also holds for any $C^{\prime} \supseteq C$.

If $R \neq[r]$, let $j \in[r] \backslash R$, and consider $R^{+}=R \cup\{j\}$. The set $\left\{\left.\mathbf{x}_{i}\right|_{C}: i \in R^{+}\right\}$is linearly dependent. Hence a unique linear combination holds for some $c_{i} \in \mathbb{F}(i \in R)$,

$$
\left.\mathbf{x}_{j}\right|_{C}=\left.\sum_{i \in R} c_{i} \mathbf{x}_{i}\right|_{C} .
$$


For any $k \notin C, \quad\left\{\left.\mathbf{x}_{i}\right|_{C \cup\{k\}}: i \in R^{+}\right\}$is also linearly dependent, and we have $\left.\mathbf{x}_{j}\right|_{C \cup\{k\}}=$ $\left.\sum_{i \in R} c_{i}^{\prime} \mathbf{x}_{i}\right|_{C \cup\{k\}}$ for some $c_{i}^{\prime} \in \mathbb{F}$. Compared to (A.1), $c_{i}^{\prime}=c_{i}$ for all $i \in R$. Hence $\mathbf{x}_{j}=\sum_{i \in R} c_{i} \mathbf{x}_{i}$, a contradiction to $\left\{\mathbf{x}_{1}, \ldots, \mathbf{x}_{r}\right\}$ being linearly independent. So $R=[r]$. There exists a non-zero $r \times r$ minor in the $R \times C$ submatrix.

For $\mathbf{x}=\left(x_{i}\right)_{i \in \mathcal{I}} \in \mathbb{F}^{\mathcal{I}}$ and $h=\left(h_{i}\right)_{i \in \mathcal{I}} \in \bigoplus_{\mathcal{I}} \mathbb{F}$ (in a direct sum, only finitely many $h_{i}$ are zero), we denote their dot product by $\mathbf{x}(h)=\sum_{i \in \mathcal{I}} x_{i} h_{i} \in \mathbb{F}$. Here we view $\mathbb{F}^{\mathcal{I}}$ as the dual space of $\bigoplus_{\mathcal{I}} \mathbb{F}$. (In general the dot product of the pair $\mathbf{x}, \mathbf{y} \in \mathbb{F}^{\mathcal{I}}$ is not defined.)

Lemma A.2. Let $\boldsymbol{x}_{1}, \ldots, \boldsymbol{x}_{r} \in \mathbb{F}^{\mathcal{I}}$ be linearly independent. Then there exist $h_{1}, \ldots, h_{r} \in \bigoplus_{\mathcal{I}} \mathbb{F}$ dual to $\boldsymbol{x}_{1}, \ldots, \boldsymbol{x}_{r}$, i.e. $\boldsymbol{x}_{i}\left(h_{j}\right)=\delta_{i j}, 1 \leq i, j \leq r$.

Proof. By Lemma A.1, there exist $r$ distinct indices $k_{j} \in \mathcal{I}, 1 \leq j \leq r$ such that the matrix $A=$ $\left(a_{i j}\right)_{i, j=1}^{r}=\left(\left(\mathbf{x}_{i}\right)_{k_{j}}\right)_{i, j=1}^{r}$ is invertible, and let $B=\left(b_{i j}\right)=A^{-1}$. Taking $h_{i}=\sum_{j=1}^{r} b_{j i} e_{k_{j}} \in \bigoplus_{\mathcal{I}} \mathbb{F}, 1 \leq$ $i \leq r$, we see that the equality $A B=I_{r}$ directly translates into the desired result.

Lemma A.3. Let $\boldsymbol{x}_{1}, \ldots, \boldsymbol{x}_{r} \in \mathbb{F}^{\mathcal{I}}$. Consider the linear map $\Phi: \bigoplus_{\mathcal{I}} \mathbb{F} \rightarrow \mathbb{F}^{r}, h \mapsto$ $\left(\boldsymbol{x}_{1}(h), \ldots, \boldsymbol{x}_{r}(h)\right)$. Then $\operatorname{dim}\left(\bigoplus_{\mathcal{I}} \mathbb{F} / \operatorname{ker} \Phi\right)=\operatorname{dim} \operatorname{span}\left\{\boldsymbol{x}_{i}\right\}_{i=1}^{r}$.

Proof. By the First Isomorphism Theorem for vector spaces $\bigoplus_{I} \mathbb{F} / \operatorname{ker} \Phi \cong \operatorname{im} \Phi$. So it suffices to prove $\operatorname{dim} \operatorname{im} \Phi=\operatorname{dim} \operatorname{span}\left\{\mathbf{x}_{i}\right\}_{i=1}^{r}$. Clearly it suffices to prove the case when $\mathbf{x}_{1}, \ldots, \mathbf{x}_{r}$ are linearly independent, and that follows directly from Lemma A.2.

Lemma A.4. Let $r \geq 0$, and let $\boldsymbol{x}_{1}, \ldots, \boldsymbol{x}_{r} \in \mathbb{F}^{\mathcal{I}}$ be $r$ linearly independent vectors and $a_{1}, \ldots, a_{r} \in$ $\mathbb{F} \backslash\{0\}$. Then for any integer $n \geq 2$, the symmetric tensor

$$
A=\sum_{i=1}^{r} a_{i} x_{i}^{\otimes n} \in \operatorname{Sym}^{n}\left(\mathbb{F}^{\mathcal{I}}\right)
$$

has $r k_{\mathrm{S}}(A)=r$. For $n \geq 3$, any expression of $A$ as $\sum_{i=1}^{r} b_{i} y_{i}^{\otimes n}$ is a permutation of the sum in (A2).

Proof. When $r=0$, the statement is trivially true so we assume $r \geq 1$. Let $n \geq 2$ and $\operatorname{rk}_{\mathrm{S}}(A)=s$. Clearly $s \leq r$. By being of symmetric rank $s$, there exist $\mathbf{y}_{1}, \ldots, \mathbf{y}_{s} \in \mathbb{F}^{\mathcal{I}}$ and $b_{1}, \ldots, b_{s} \in \mathbb{F} \backslash\{0\}$ such that

$$
\sum_{i=1}^{r} a_{i} \mathbf{x}_{i}^{\otimes n}=A=\sum_{j=1}^{s} b_{j} \mathbf{y}_{j}^{\otimes n} .
$$

By Lemma A.2 there exist $h_{1}, \ldots, h_{r}$ dual to $\mathbf{x}_{1}, \ldots, \mathbf{x}_{r}$. For any $1 \leq i \leq r$, applying $h_{i}^{\otimes(n-1)}$ to the sum, we get $a_{i} \mathbf{x}_{i}$ as a linear combination of $\mathbf{y}_{1}, \ldots, \mathbf{y}_{s}$. Hence $s \geq r$ as $\mathbf{x}_{1}, \ldots, \mathbf{x}_{r}$ are linearly independent. So $s=r$, and $\mathbf{y}_{1}, \ldots, \mathbf{y}_{s}$ are linearly independent.

Next, let $n \geq 3$ and consider (A.3) again, where $s=r$. Since $\operatorname{rks}_{S}(A)=r$, all $b_{j} \neq 0$. Applying $h_{i}$, we get

$$
a_{i} \mathbf{x}_{i}^{\otimes(n-1)}=B=\sum_{j=1}^{r} b_{j} \mathbf{y}_{j}\left(h_{i}\right) \mathbf{y}_{j}^{\otimes(n-1)} .
$$

From the LHS, $\operatorname{rk}_{\mathrm{S}}(B)=1$. By what has just been proved, $\operatorname{rk}_{\mathrm{S}}(B)$ is the number of terms with nonzero coefficients on the RHS. Hence for any $i$, there is exactly one $j$ such that $\mathbf{y}_{j}\left(h_{i}\right) \neq 0$. Applying $h_{i}^{\otimes(n-2)}$ to (A.4), we get $a_{i} \mathbf{x}_{i}=b_{j}^{\prime} \mathbf{y}_{j}$, where $b_{j}^{\prime}=b_{j}\left(\mathbf{y}_{j}\left(h_{i}\right)\right)^{n-1} \neq 0$. Since $\mathbf{x}_{1}, \ldots, \mathbf{x}_{r}$ are linearly independent, the map $i \mapsto j$ is a permutation. From $a_{i} \mathbf{x}_{i}=b_{j}^{\prime} \mathbf{y}_{j}$ we get $a_{i}=b_{j}^{\prime} \mathbf{y}_{j}\left(h_{i}\right)=b_{j}\left(\mathbf{y}_{j}\left(h_{i}\right)\right)^{n}$. It follows that $\mathbf{y}_{j}=\left(a_{i} / b_{j}^{\prime}\right) \mathbf{x}_{i}=\mathbf{y}_{j}\left(h_{i}\right) \mathbf{x}_{i}$. Therefore $b_{j} \mathbf{y}_{j}^{\otimes n}=b_{j}\left(\mathbf{y}_{j}\left(h_{i}\right)\right)^{n} \mathbf{x}_{i}^{\otimes n}=a_{i} \mathbf{x}_{i}^{\otimes n}$. Thus the expressions on the LHS and the RHS of (A.3) are the same up to a permutation of the terms. 
Lemma A.5. Let $r \geq 0$, and let $\boldsymbol{x}_{1}, \ldots, \boldsymbol{x}_{r} \in \mathbb{F}^{\mathcal{I}}$ be $r$ non-zero pairwise linearly independent vectors. Then for any non-negative integer $n \geq r-1$, the rank-1 symmetric tensors

$$
\boldsymbol{x}_{1}^{\otimes n}, \ldots, \boldsymbol{x}_{r}^{\otimes n} \in \operatorname{Sym}^{n}\left(\mathbb{F}^{\mathcal{I}}\right)
$$

are linearly independent.

Proof. The case $r=0$ is vacuously true. It is also trivially true for $r=1$, since $\mathbf{x}_{1}^{\otimes n}$ is nonzero. Assume $r \geq 2$. By pairwise linear independence, for every $1 \leq i, j \leq r, i \neq j$, from Lemma A.2 there exists $h_{i j}$ such that $\mathbf{x}_{i}\left(h_{i j}\right)=1, \mathbf{x}_{j}\left(h_{i j}\right)=0$. Suppose $\sum_{i=1}^{r} \lambda_{i} \overline{\mathbf{x}}_{i}^{\otimes n}=0$ where $\lambda_{i} \in \mathbb{F}, 1 \leq i \leq r$. Applying $\bigotimes_{1 \leq j \leq r, j \neq i} h_{i j}$, we get $\lambda_{i} \mathbf{x}_{i}^{\otimes(n-(r-1))}=0$ for $1 \leq i \leq r$, and thus $\lambda_{i}=0$ since $n \geq r-1$ and therefore $\mathbf{x}_{i}^{\otimes(n-(r-1))}$ is non-zero. Hence $\mathbf{x}_{1}^{\otimes n}, \ldots, \mathbf{x}_{r}^{\otimes n}$ are linearly independent.

Remark A.1. For $r \geq 2$, the non-zero hypothesis is subsumed by pairwise linear independence.

\section{A.2 Finite symmetric tensor rank}

The proof of the following lemma is essentially the same as Lemma 4.2 in [20]; the only modification needed is to avoid a symmetrization step, which could result in a division by 0 in a field of finite characteristic.

Lemma A.6. If $\mathbb{F}$ is a field of cardinality $|\mathbb{F}|>n$, a fortiori if $\mathbb{F}$ is infinite, and $V$ is a vector space over $\mathbb{F}$, then every symmetric tensor $A \in \operatorname{Sym}^{n}(V)$ has a finite symmetric tensor rank $r k_{\mathrm{S}}(A)<\infty$. Moreover, when $\operatorname{dim} V<\infty$, we have $r_{\mathrm{S}}(A) \leq\left(\begin{array}{c}\operatorname{dim} V+n-1 \\ n\end{array}\right)$.

Proof. By definition, every $A \in \operatorname{Sym}^{n}(V) \subseteq V^{\otimes n}$ is a finite sum $A=\sum_{i=1}^{m} v_{i 1} \otimes \cdots \otimes v_{i n}$. Let $V^{\prime}=\operatorname{span}\left\{v_{i j} \mid i \in[m], j \in[n]\right\}$ be a finite dimensional subspace of $V$. As $A \in \operatorname{Sym}^{n}\left(V^{\prime}\right)$, we can assume $V$ is finite dimensional, with no change in $\operatorname{rk}_{S}(A)$; so we let $V=\mathbb{F}^{N}$, for some $N$.

Let $T=\operatorname{span}\left\{x^{\otimes n} \mid x \in V\right\} \subseteq \operatorname{Sym}^{n}(V)$. Our claim is that equality holds. For every entry of $x^{\otimes n}$, which is a product of coordinate entries of $x$, we can classify it by how many factors are the $j$ th coordinate of $x$, for $j \in[N]$. There are $\left(\begin{array}{c}n+N-1 \\ N-1\end{array}\right)=\left(\begin{array}{c}N+n-1 \\ n\end{array}\right)$ coordinates which can be indexed by tuples $\left(i_{1}, \ldots, i_{N}\right)$ where $i_{1}, \ldots, i_{N} \geq 0$ and $i_{1}+\cdots+i_{N}=n$, such that every entry of every $t \in T$ is equal to its entry at one of these coordinates. We define a compression operator $C$ which selects only those $\left(\begin{array}{c}N+n-1 \\ n\end{array}\right)$ coordinates, and define

$$
S=\operatorname{span}\left\{C\left(x^{\otimes n}\right) \mid x \in V\right\}=\{C(t) \mid t \in T\} .
$$

The compression operator $C$ is also applicable to $\operatorname{Sym}^{n}(V)$. Indeed, by definition as a symmetric array in $\mathbb{F}^{V^{n}}$, any $A \in \operatorname{Sym}^{n}(V)$ is invariant under any permutation of $n$. This means that for any $\left(k_{1}, \ldots, k_{n}\right)$, where $k_{1}, \ldots, k_{n} \in[N]$, and any permutation $\pi \in S_{n}$, if $\left(e_{1}^{*}, \ldots, e_{N}^{*}\right)$ is the dual basis to the canonical basis of $V$, then $\left(e_{k_{1}}^{*} \otimes \cdots \otimes e_{k_{n}}^{*}\right)(A)=\left(e_{k_{\pi(1)}}^{*} \otimes \cdots \otimes e_{k_{\pi(n)}}^{*}\right)(A)$. This invariance can be characterized by the tuple $\left(i_{1}, \ldots, i_{N}\right)$, where $i_{j}=$ the number $j \in[N]$ among $\left(k_{1}, \ldots, k_{n}\right)$. Thus $C$ is applicable to $\operatorname{Sym}^{n}(V)$, and we denote the result $C\left(\operatorname{Sym}^{n}(V)\right)=\left\{C(v) \mid v \in \operatorname{Sym}^{n}(V)\right\} \subseteq$ $\mathbb{F}\left(\begin{array}{c}N+n-1 \\ n\end{array}\right)$. As $T \subseteq \operatorname{Sym}^{n}(V)$, we have $S \subseteq C\left(\operatorname{Sym}^{n}(V)\right)$.

Next we prove that $S=\mathbb{F}^{\left({ }^{N+n-1}\right)}{ }_{n}$. Then it follows that $S=C\left(\operatorname{Sym}^{n}(V)\right)$, from which it clearly follows that $T=\operatorname{Sym}^{n}(V)$ since $C$ simply removes repeated entries.

Suppose otherwise, then $\operatorname{dim} S<\left(\begin{array}{c}N+n-1 \\ n\end{array}\right)$. There exists a non-zero vector in $\mathbb{F}^{\left(\begin{array}{c}N+n-1 \\ n\end{array}\right)}$ such that it has a zero dot product with all $S$. This means there exists a non-zero tuple $\left.\left(a_{\left(i_{1}, \ldots, i_{N}\right)}\right) \in \mathbb{F}^{(N+n-1}\right)$ indexed by $N$-tuples of non-negative integers that sum to $n$, such that $\sum_{\left(i_{1}, \ldots, i_{N}\right)} a_{\left(i_{1}, \ldots, i_{N}\right)} x_{1}^{i_{1}} \cdots x_{N}^{i_{N}}=0$, for all $x_{1}, \ldots, x_{N} \in \mathbb{F}$. 
As a polynomial in $x_{N}$ it has degree at most $n$, and yet it vanishes at $|\mathbb{F}|>n$ points. So for any fixed $0 \leq i_{N} \leq n, \sum_{\left(i_{1}, \ldots, i_{N-1}\right)} a_{\left(i_{1}, \ldots, i_{N-1}, i_{N}\right)} x_{1}^{i_{1}} \cdots x_{N-1}^{i_{N-1}}=0$, for all $x_{1}, \ldots, x_{N-1} \in \mathbb{F}$, which can be viewed as a polynomial in $x_{N-1}$ of degree at most $n-i_{N} \leq n$. Iterating $N$ steps, we reach a contradiction that the tuple $\left(a_{\left(i_{1}, \ldots, i_{N}\right)}\right)$ is entirely zero.

\section{A.3 Subalgebras of $\mathbb{F}^{m}$}

We give a proof of Lemma 5.3, restated below.

Lemma A.7. All subalgebras of $\mathbb{F}^{m}$, where $m \geq 0$, are of the following form: For some partition $[m]=\bigsqcup_{i=1}^{s} \mathcal{I}_{i}$, where $s \geq 0$, and $\mathcal{I}_{i} \neq \emptyset$ for $i \in[s]$, the subalgebra has equal values on each $I_{i}$,

$$
\mathbb{F}^{\left(\mathcal{I}_{1}, \ldots, \mathcal{I}_{s}\right)}=\left\{\left(c_{1}, \ldots, c_{m}\right) \in \mathbb{F}^{m} \mid \forall i \in[s], \forall j, j^{\prime} \in \mathcal{I}_{i}, c_{j}=c_{j^{\prime}}\right\} .
$$

Proof. When $m=0$, the statements is obvious. Let $m \geq 1$ and $S \subseteq \mathbb{F}^{m}$ be a subalgebra of $\mathbb{F}^{m}$. In particular, the multiplicative identity is the $m$-tuple $(1, \ldots, 1) \in S$. We call $i, j \in[m]$ equivalent if $x_{i}=x_{j}$ for any $x=\left(x_{1}, \ldots, x_{m}\right) \in S$. This is clearly an equivalence relation so it partitions $[m]$ into (non-empty) equivalence classes $\mathcal{I}_{1}, \ldots, \mathcal{I}_{s}$ so that $[m]=\bigsqcup_{i=1}^{s} \mathcal{I}_{i}$. As $[m] \neq \emptyset$ we have $s \geq 1$. We claim that $S=\mathbb{F}^{\left(\mathcal{I}_{1}, \ldots, \mathcal{I}_{s}\right)}$. Clearly, $S \subseteq \mathbb{F}^{\left(\mathcal{I}_{1}, \ldots, \mathcal{I}_{s}\right)}$. We prove the reverse inclusion. For $s=1$ this is clearly true since the $m$-tuple $(1, \ldots, 1) \in S$ and $S$ is closed under scalar multiplication.

Now we let $s \geq 2$. By renaming and omitting repeated coordinates it is sufficient to prove the case when $m=s$ and $\mathcal{I}_{i}=\{i\}$. Let $S^{\prime}=\left\{\left(c_{2}, \ldots, c_{s}\right) \mid \exists c_{1} \in \mathbb{F},\left(c_{1}, c_{2}, \ldots, c_{s}\right) \in S\right\}$ be the projection of $S$ to $\mathbb{F}^{s-1}$. Clearly $S^{\prime}$ is a subalgebra of $\mathbb{F}^{s-1}$, and by induction $S^{\prime}=\mathbb{F}^{s-1}$. Thus for some $b_{1}, \ldots, b_{s-1} \in \mathbb{F}$, all $s-1$ row vectors of the following $(s-1) \times s$ matrix $B$ belong to $S$,

$$
B=\left[\begin{array}{ccccc}
b_{1} & 1 & 0 & \ldots & 0 \\
b_{2} & 0 & 1 & \ldots & 0 \\
\vdots & \vdots & \vdots & \ddots & \vdots \\
b_{s-1} & 0 & 0 & \ldots & 1
\end{array}\right]
$$

If all $b_{i}=0$, we let $v=(1,1, \ldots, 1) \in S$. Otherwise we may assume $b_{1} \neq 0$. By the definition of $I_{1}$ and $I_{2}$ and by the closure of $S$ under scalar product and possibly adding $(1,1, \ldots, 1)$, for some $c_{1} \neq 1$ and for some $c_{3}, \ldots, c_{s} \in \mathbb{F}$, we have $v^{\prime}=\left(c_{1}, 1, c_{3}, \ldots, c_{s}\right) \in S$. Then multiplying $v^{\prime}$ with the first row in (A.5) we get $v=\left(b_{1} c_{1}, 1,0, \ldots, 0\right) \in S$. Here $b_{1} c_{1} \neq b_{1}$. In either case, we obtain a matrix $A$ of rank $s$ by appending $v$ as the last row to $B$. Thus for all row vectors $d \in \mathbb{F}^{s}$ the linear system $x A=d$ has a solution $x \in \mathbb{F}^{s}$. This shows that $d \in S$.

\section{Acknowledgements}

The authors would like to thank the anonymous referees of both the journal submission and the 30th Annual ACM-SIAM Symposium on Discrete Algorithms (SODA) 2019 for very helpful comments. Their suggestions have improved and clarified many points. We particularly thank one referee to point out the paper [51] by Balázs Szegedy, and also the expressibility of the hardcore gas model on $d$-regular graphs as a partition function of $\mathrm{GH}$.

\section{References}

[1] Asano, T. (1970) Theorems on the partition functions of the Heisenberg ferromagnets. J. Phys. Soc. Japan $29350-359$.

[2] Atserias, A., Kolaitis, P. G. and Wu, W.-L. (2021) On the expressive power of homomorphism counts. arXiv:2101.12733. 
[3] Backens, M. (2017) A new Holant dichotomy inspired by quantum computation. In Proceedings of the 44th International Colloquium on Automata, Languages, and Programming (ICALP), pp. 16:1-16:14. Full version available at https://arxiv.org/abs/1702.00767.

[4] Backens, M. (2018) A complete dichotomy for complex-valued Holant ${ }^{c}$. In Proceedings of the 45th International Colloquium on Automata, Languages, and Programming (ICALP), pp. 12:1-12:14. Full version available at https://arxiv.org/abs/1704.05798.

[5] Baxter, R. J. (2004) The six and eight-vertex models revisited. J. Stat. Phys. 116 43-66.

[6] Borgs, C., Chayes, J., Lovász, L., Sós, V. T. and Vesztergombi, K. (2006) Counting graph homomorphisms. In Topics in Discrete Mathematics, Springer, Berlin, Heidelberg, pp. 315-371.

[7] Borgs, C., Chayes, J. T., Lovász, L., Sós, V. T. and Vesztergombi, K. (2008) Convergent sequences of dense graphs I: Subgraph frequencies, metric properties and testing. Adv. Math. 219 1801-1851.

[8] Bulatov, A. (2013) The complexity of the counting constraint satisfaction problem. J. ACM 60 34:1-34:41.

[9] Bulatov, A. and Grohe, M. (2005) The complexity of partition functions. Theor. Comput. Sci. 348 148-186. A preliminary version appeared in ICALP 2004: 294-306.

[10] Bulatov, A. A. and Dalmau, V. (2007) Towards a dichotomy theorem for the counting constraint satisfaction problem. Inf. Comput. 205 651-678.

[11] Cai, J.-Y. and Chen, X. (2017a) Complexity Dichotomies for Counting Problems, Volume 1: Boolean Domain, Cambridge University Press. https://doi.org/10.1017/9781107477063.

[12] Cai, J.-Y. and Chen, X. (2017b) Complexity of counting CSP with complex weights. J. ACM 64 19:1-19:39.

[13] Cai, J.-Y., Chen, X. and Lu, P. (2013) Graph homomorphisms with complex values: A dichotomy theorem. SIAM J. Comput. 42 924-1029.

[14] Cai, J.-Y. and Fu, Z. (2019) Holographic algorithm with matchgates is universal for planar \#CSP over Boolean domain. SIAM J. Comput. 0 STOC17-50-STOC17-151.

[15] Cai, J.-Y., Fu, Z., Guo, H. and Williams, T. (2015) A Holant dichotomy: Is the FKT algorithm universal? In Proceedings of the 56st Annual IEEE Symposium on Foundations of Computer Science (FOCS), pp. 1259-1276.

[16] Cai, J.-Y. and Govorov, A. (2019) Perfect matchings, rank of connection tensors and graph homomorphisms. In Proceedings of the 30th Annual ACM-SIAM Symposium on Discrete Algorithms (SODA), pp. 476-495.

[17] Cai, J.-Y. and Govorov, A. (2021) On a theorem of Lovász that hom $(\cdot, H)$ determines the isomorphism type of $H$. ACM Trans. Comput. Theory 13 11:1-11:25.

[18] Cai, J.-Y., Lu, P. and Xia, M. (2009) Holant problems and counting CSP. In Proceedings of the 41st Annual ACM Symposium on Theory of Computing (STOC), pp. 715-724.

[19] Cohn, P. M. (1991) Algebra, 2nd edn., Vol. 3, John Wiley \& Sons.

[20] Comon, P., Golub, G., Lim, L.-H. and Mourrain, B. (2008) Symmetric tensors and symmetric tensor rank. SIAM J. Matrix Anal. Appl. 30 1254-1279.

[21] Dell, H., Grohe, M. and Rattan, G. (2018) Lovász meets Weisfeiler and Leman. In Proceedings of the 45th International Colloquium on Automata, Languages and Programming (ICALP), pp. 40:1-40:14.

[22] Draisma, J., Gijswijt, D. C., Lovász, L., Regts, G. and Schrijver, A. (2012) Characterizing partition functions of the vertex model. J. Algebra 350 197-206.

[23] Dvořák, Z. (2010) On recognizing graphs by numbers of homomorphisms. J. Graph Theory 64 330-342.

[24] Dyer, M. E. and Greenhill, C. S. (2000) The complexity of counting graph homomorphisms. Random Struct. Algorithms 17 260-289. A preliminary version appeared in SODA 2000: 246-255.

[25] Dyer, M. E. and Greenhill, C. S. (2004) Corrigendum: The complexity of counting graph homomorphisms. Random Struct. Algorithms 25 346-352.

[26] Dyer, M. E. and Richerby, D. (2013) An effective dichotomy for the counting constraint satisfaction problem. SIAM J. Comput. 42 1245-1274.

[27] Freedman, M., Lovász, L. and Schrijver, A. (2007) Reflection positivity, rank connectivity, and homomorphism of graphs. J. Am. Math. Soc. 20 37-51.

[28] Goldberg, L. A., Grohe, M., Jerrum, M. and Thurley, M. (2010) A complexity dichotomy for partition functions with mixed signs. SIAM J. Comput. 39 3336-3402.

[29] Gómez, C., Ruiz-Altaba, M. and Sierra, G. (1996) Quantum Groups in Two-Dimensional Physics, Cambridge Monographs on Mathematical Physics, Cambridge University Press.

[30] Grohe, M. and Thurley, M. (2011) Counting homomorphisms and partition functions. In Model Theoretic Methods in Finite Combinatorics, Vol. 558 of Contemporary Mathematics (M. Grohe and J. Makowsky, eds), American Mathematical Society, pp. 243-292.

[31] Guo, H., Lu, P. and Valiant, L. G. (2013) The complexity of symmetric Boolean parity Holant problems. SIAM J. Comput. 42 324-356.

[32] Hell, P. and Nešetřil, J. (2004) Graphs and Homomorphisms, Vol. 28 of Oxford Lecture Series in Mathematics and its Applications, Oxford University Press.

[33] Kasteleyn, P. W. (1961) The statistics of dimers on a lattice: I. The number of dimer arrangements on a quadratic lattice. Physica 27 1209-1225.

[34] Kasteleyn, P. W. (1967) Graph theory and crystal physics. In Graph Theory and Theoretical Physics (F. Harary, ed), pp. 43-110. 
[35] Lee, T.-D. and Yang, C.-N. (1952) Statistical theory of equations of state and phase transitions. II. lattice gas and Ising model. Phys. Rev. 87 410-419.

[36] Lieb, E. H. and Sokal, A. D. (1981) A general Lee-Yang theorem for one-component and multicomponent ferromagnets. Commun. Math. Phys. 80 153-179.

[37] Lovász, L. (1967) Operations with structures. Acta Math. Hungar. 18 321-328.

[38] Lovász, L. (2006) The rank of connection matrices and the dimension of graph algebras. Eur. J. Comb. $27962-970$.

[39] Lovász, L. (2012) Large Networks and Graph Limits, Vol. 60 of Colloquium Publications, American Mathematical Society.

[40] Lovász, L. and Schrijver, A. (2008) Graph parameters and semigroup functions. Eur. J. Comb. 29 987-1002.

[41] Lovász, L. and Sós, V. T. (2008) Generalized quasirandom graphs. J. Comb. Theory Ser. B 98 146-163.

[42] Lovász, L. and Szegedy, B. (2006) Limits of dense graph sequences. J. Comb. Theory Ser. B 96 933-957.

[43] Lovász, L. and Szegedy, B. (2009) Contractors and connectors of graph algebras. J. Graph Theory 60 11-30.

[44] Newman, C. M. (1974) Zeros of the partition function for generalized Ising systems. Commun. Pure Appl. Math. 27 143-159.

[45] Regts, G. (2013) Graph Parameters and Invariants of the Orthogonal Group. PhD thesis, Universiteit van Amsterdam.

[46] Schrijver, A. (2009) Graph invariants in the spin model. J. Comb. Theory Ser. B 99 502-511.

[47] Schrijver, A. (2012) Characterizing partition functions of the vertex model by rank growth. arXiv:1211.3561v1.

[48] Schrijver, A. (2013) Characterizing partition functions of the spin model by rank growth. Indagationes Mathematicae 24 1018-1023.

[49] Schrijver, A. (2015) Characterizing partition functions of the edge-coloring model by rank growth. J. Comb. Theory Ser. A 136 164-173.

[50] Szegedy, B. (2007) Edge coloring models and reflection positivity. J. Amer. Math. Soc. 20 969-988.

[51] Szegedy, B. (2010) Edge Coloring Models as Singular Vertex Coloring Models, Bolyai Society Mathematical Studies, Springer, Berlin, Heidelberg, pp. 327-336.

[52] Temperley, H. N. V. and Fisher, M. E. (1961) Dimer problem in statistical mechanics-an exact result. Philos. Mag. 6 1061-1063.

[53] Thurley, M. (2009) The Complexity of Partition Functions. PhD thesis, Humboldt Universität zu Berlin.

[54] Valiant, L. G. (2006) Accidental algorithms. In Proceedings of the 47th Annual IEEE Symposium on Foundations of Computer Science (FOCS), pp. 509-517.

[55] Valiant, L. G. (2008) Holographic algorithms. SIAM J. Comput. 37 1565-1594. A preliminary version appeared in FOCS 2004: 306-315.

Cite this article: Cai J-Y and Govorov A (2022). Perfect matchings, rank of connection tensors and graph homomorphisms. Combinatorics, Probability and Computing 31, 268-303. https://doi.org/10.1017/S0963548321000286 